\title{
ACMT 2019 Annual Scientific Meeting Abstracts-San Francisco, CA
}

\begin{abstract}
These are the abstracts of the 2019 American College of Medical Toxicology (ACMT) Annual Scientific Meeting. Included here are $162 \mathrm{ab}-$ stracts that will be presented in April 2019 in San Francisco, California, including research studies from around the globe and the ToxIC collaboration, clinically significant case reports describing new toxicologic phenomena, and encore research presentations from other scientific meetings.
\end{abstract}

Keywords: Abstracts-Annual Scientific Meeting-Toxicology Investigators Consortium-Medical Toxicology Foundation-Pediatric Environmental Health Specialty Units

Correspondence: American College of Medical Toxicology (ACMT) 10645 N. Tatum Blvd Phoenix, AZ; info@acmt.net

Introduction: The American College of Medical Toxicology (ACMT) received 212 eligible research abstracts for consideration for presentation at the 2019 Annual Scientific Meeting (ASM), including 96 research studies and 116 case reports. Each abstract was reviewed in a blinded fashion by at least four Research Committee members. Each abstract was independently scored based on the clinical question, data source, analytic method, results/conclusion, and clarity of presentation. A total of 162 abstracts from a competitive pool were accepted for presentation to this year's Annual Scientific Meeting. This work would not be possible without the hard work and diligence of our abstract reviewers: Ann Arens, Justin Arnold, Katie Boyle, Nick Brandehoff, Jeffrey Brent, Stephanie Carreiro, James Chenoweth, Neeraj Chhabra, Jon Cole, Nick Connors, Kirk Cumpston, Rob Hendrickson, David Jang, David Juurlink, Louise Kao, Ken Katz, Katherine Katzung, Russ Kerns, Andrew King, Kathy Kopec, JoAn Laes, Eric Lavonas, Michael Levine, Heather Long, Joe Maddry, Kevin Maskell, Maryann Mazer-Amirshahi, William Meggs, Elissa Moore, Mark Mycyk, Matt Noble, Travis Olives, Renee Petzel Gimbar, Evan Schwarz, Daniel Sessions, Sam Stellpflug, Mark Su, Manoj Tyagi, Shawn Varney, Steven Walsh, Richard Wang, Tim Wiegand, Sage Wiener, Brandon Wills, and Luke Yip. Equally significant is the contribution of the ACMT staff (Lizzy Nguyen and Adrienne Dunavin) who led the process. Congratulations to all the contributors whose work will be presented in San Francisco.

We look forward to seeing you there.

Shawn M. Varney, MD, FACMT, Abstract Review Chair; Jon B. Cole, MD, FACMT, Abstract Review Co-Chair, Maryann Mazer-Amirshahi, PharmD, MD, MPH, Chair, ACMT Research Committee

\section{Day 1: Platforms, Abstracts 001-004}

\section{Bupropion-Associated Seizures Following an Acute Overdose}

Steve Offerman ${ }^{1}$, Jasmin Goshen ${ }^{2}$, Angela Padilla-Jones ${ }^{3}$, AnneMichelle Ruha ${ }^{3,4}$, Stephen Thomas ${ }^{5}$, Michael Levine ${ }^{6}$

${ }^{1}$ Medical Toxicology Consultation Service. Kaiser Permanente Northern California, Sacramento, CA. ${ }^{2}$ California North State University College of Medicine, Elk Grove, CA. ${ }^{3}$ Banner-University Medical Center, Phoenix, Phoenix, AZ. ${ }^{4}$ University of Arizona College of Medicine, Phoenix, AZ. ${ }^{5}$ Weill Cornell College of Medicine in Qatar and Hamad Medical Corporation, Doha, Qatar. ${ }^{6}$ University of Southern California, Los Angeles, CA

Background: Patients with bupropion overdose are routinely observed for prolonged periods due to concerns for delayed seizures. The study sought to evaluate characteristics of bupropion ingestions and attempt to determine an appropriate observation period.

Hypothesis: Patients with bupropion toxicity will have antecedent altered mental status (AMS) and/or tachycardia before seizure.

Methods: This multicenter, retrospective study, utilized standardized data abstraction methods, included all patients who presented with a bupropion ingestion to 1 health system ( 20 hospitals), 1 toxicology practice (5 hospitals), and toxicology referral center. Data collected included demographics, ingestion history (time, dose, preparation), clinical characteristics (vital signs, seizures, AMS) length of stay, and treatment. Medians (IQR) were utilized for descriptive statistics, Chi-square, and/or Fisher's exact for categorical variables. Logistic regression was performed to assess for confounders. The following definitions were used: delayed seizure (first seizure $>8 \mathrm{~h}$ post arrival); persistent tachycardia (tachycardia lasting $>2 \mathrm{~h}$ ).

Results: Four hundred thirty-seven encounters were identified. The median (IQR) age was 28 (18-43) years; 275 (63\%) were female. Seventy-eight percent of cases involved intentional exposures. Accidental double-dose ingestions accounted for 39 (8.9\%) cases. Seizures occurred in $122(27.9 \%)$ subjects (68 pre-hospital seizures, 75 in-hospital seizures). The median (IQR) length of stay was 36 (12-72) hours. Using logistic regression, the tachycardia or AMS at arrival were each associated with an increased odds of seizure (OR 3.98 [95\% CI 2.2-7.3] for tachycardia; OR 2.65, [95\% CI 2.18-7.26] for altered mental status). Only 1 of 143 subjects who arrived without tachycardia or AMS had a delayed seizure $(0.7 \%$; $95 \%$ CI $0.02-$ $3.9 \%$ ). Of eight cases with delayed seizures, all had persistent tachycardia prior to the seizure.

Conclusion: Seizures are common following bupropion overdose and are predicted by tachycardia or AMS. Seizures beyond $8 \mathrm{~h}$ of observation are unusual and were accompanied by antecedent tachycardia and/or AMS.

002. Fentanyl Analog Exposures Among Living Patients in a Large Urban Healthcare System

Neeraj Chhabra ${ }^{1,2}$, Lum Rizvanolli ${ }^{1}$, Arkady Rasin ${ }^{1,2}$, Granger Marsden ${ }^{1}$, Keiki Hinami ${ }^{1}$, Steven E Aks ${ }^{1,2}$

${ }^{1}$ Cook County Health, Chicago, IL, USA. ${ }^{2}$ Toxikon Consortium, Chicago, IL, USA

Background: Fentanyl contamination of illicit drugs is a growing concern in the USA with an increasing mortality rate resulting from synthetic opioid exposures. The extent to which such contamination is driven by potentially more potent fentanyl analogs 
remains unclear. The majority of data regarding fentanyl analog exposure has originated from medical examiner offices and law enforcement seizures, with little information regarding exposures in living patients.

Research Question: For patients screening positive for illicit opioids or fentanyl, are there also detectable fentanyl analogs on a reference fentanyl analog panel?

Methods: This is a retrospective analysis of urine opioid testing results from patients in an urban public healthcare system from May through July 2018. A convenience sample of patients' urine samples which screened positive for opioids or fentanyl on ELISA assay was referred to a reference laboratory (NMS Labs, Willow Grove PA) for identification of fentanyl analogs by highperformance liquid chromatography/tandem mass spectroscopy (HPLC/MS). HPLC/MS was performed on specimens for the identification of 20 "designer opioids" including carfentanil, acryl fentanyl, and 4-ANPP.

Results: A total of 142 urine samples were referred for fentanyl analog testing. Sixty-two samples were referred due to detection of both opioids and fentanyl, 31 for opioids alone, and 49 for fentanyl alone. Fentanyl analogs were detected in 86 samples $(60.6 \%)$. The median number of analogs detected per sample was 1 (range $0-5$ ). The most common analogs detected were 4-ANPP, 78; 2furanylfentanyl, 18; cyclopropylfentanyl, 14; acryl fentanyl, 13; and carfentanil, 10

Conclusion: "Fentanyl contamination" is not restricted strictly to fentanyl but also its analogs which may be more potent. Testing samples from living patients provides a useful mechanism to detect exposure patterns as well as identify patients at risk of overdose death and target resources for evidence-based interventions such as prescription-naloxone and medication-assisted therapy.

\section{Preliminary Findings of False-Positive Interferences of Lidocaine for Cocaine Metabolite in a Urine Drug Screen Immunoassay}

Eungjae Kim, Brian Murray, Tim Moran, Joseph Carpenter, David Koch, James Ritchie, Joanna Schindler, Brent Morgan

Emory University, Atlanta, Georgia, USA

Background: Individuals accused of testing positive for cocaine have claimed that lidocaine, or its metabolite monoethylglycinexylidide (MEG-X), may cause false-positive results for the cocaine metabolite, benzoylecgonine (BE). After a thorough research of peer-reviewed medical literature, we have found no studies that have investigated this claim.

Methods: The goal of the study was to determine if lidocaine exposure for routine medical procedures results in false-positive findings on a commercially available immunoassay urine drug screen (UDS). We performed a cross-sectional observational study of patients receiving lidocaine as part of their regular care. Standard immunoassay drug screens and confirmatory liquid chromatography-mass spectrometry (LC-MS) were performed on all urine samples to assess for MEG-X and BE. Results for categorical variables are presented as percentages with Agresti-Coull $95 \%$ confidence intervals. Continuous variables are presented as medians and interquartile ranges.

Results: In total, 112 samples were collected of which 77 tested positive for MEGX and negative for BE. The sample was $71.1 \%$ (59.9-80.1) male and the median age was 41 (IQR 29-53). Then, 80.3\% (69.8-87.8) received intradermal lidocaine in the Emergency Room/Trauma zone and $19.7 \%(12.2-30.2)$ received intravenous lidocaine in preparation for a surgery. Further, $68.9 \%(57.6-78.4)$ received $1 \%$ lidocaine, $33.8 \%$ (24.0-45.2) received $2 \%$ lidocaine, and $38.4 \%(28.0-49.8)$ received lidocaine with epinephrine. The median dose administered was $100 \mathrm{mg}$ $(\mathrm{IQR}=30-55)$. There were no false-positive UDS results in this sample $(0 \% ; 0-5.7)$.
Conclusion: In this preliminary review of samples, no false-positive readings were discovered. Thus, the current study failed to find any evidence that lidocaine, or MEGX specifically, causes false-positive results on an immunoassay for BE.

\section{Challenges in the Implementation of a Urine Immunoassay for Buprenorphine at a Children's Hospital}

Kristen McCabe, Tracey Polsky, Kevin Osterhoudt The Children's Hospital of Philadelphia, Philadelphia, PA, USA

Background: Failure to detect opioid poisoning among children may negatively affect child health, and our hospital's commercial urine drug immunoassay (Vitros5.1 Chemistry System ${ }^{\circledR}$, Ortho Clinical Diagnostics) may fail to detect the synthetic opioid buprenorphine. We implemented an additional specific immunoassay (CEDIA Buprenorphine II ${ }$, Thermo Scientific) for buprenorphine and tracked its clinical performance.

Methods: Patients with positive urine buprenorphine immunoassays from a single tertiary care children's hospital were identified prospectively during the first 28 days of test implementation (October 9-November 5, 2018). Patient characteristics were collected prospectively as part of quality assurance. Each positive sample was sent for confirmation by mass spectrometry. This dataset was de-identified then abstracted by a single, blinded investigator.

Results: One hundred ninety-eight patients had a urine drug immunoassay during the study period, and 12/129 screened positive for buprenorphine. Ages ranged 5 to 17 years (median 14); three were male and nine were female. Eleven of 12 subjects had no history of suspected pre-hospital opioid exposure, and none was administered opioids. Three of 12 urine drugs screens were performed as part of a routine psychiatry evaluation clinical practice pattern, and 9/12 were performed due to acute or subacute behavior changes. No subject was noted to have pinpoint pupils or respiratory depression, and none was given naloxone. Eleven subjects had sufficient sample for confirmatory testing, and all confirmatory tests were negative for buprenorphine and its metabolite norbuprenorphine. The false-positive rate was $6.9 \%$ [95\% CI $3.5-$ $10.4 \%$ ] and no true-positive tests were obtained; the false-negative rate was not identifiable. No two subjects were identified with a common home- or hospital-administered medication to implicate a cross-reacting drug.

Conclusion: Our implementation of a urine buprenorphine immunoassay has encountered a troubling false-positive test rate in a setting of uncertain pre-test probability. False-positive opioid testing creates care challenges; the cost-benefit of this test merits careful consideration.

\section{Day 1: Moderated Posters, Abstracts 005-010}

\section{What Dose of THC Leads to Hospital Admission?}

Robert Hendrickson ${ }^{1,2}$, Matthew Noble ${ }^{1,2}$

${ }^{1}$ Oregon Health and Science University, Portland, Oregon, USA. ${ }^{2}$ Oregon Poison Center, Portland, Oregon, USA

Background: Tetrahydrocannabinol (THC) is legal in 31 states as "medicinal cannabis" and in 9 states as "retail cannabis." States have adopted different THC doses (mg) permitted in a "serving size" (e.g., $5 \mathrm{mg}, 10 \mathrm{mg}$ ) or package (e.g., $15 \mathrm{mg}, 50 \mathrm{mg}, 100 \mathrm{mg}$ ).

Research Question: Is there a relationship between THC-dose and admission rate in patients who ingest THC edibles/concentrates?

Methods: Prospective study of subjects where the Oregon Poison Center (OPC) was contacted about cannabis exposure between 12/4/ 
2015 and 4/15/2017. A data collection sheet captured clinical and product data including THC-dose. Inclusion criteria: cannabis exposure with ingested dose recorded. Exclusion criteria: co-ingestion of other medications/drugs. Fisher's exact test was used to compare frequencies of events.

Results: We identified 34 patients. Ages ranged from 17 months to 76 years, median 25 years. Forty-four percent were female. Eightytwo percent ingested edible products; $21 \%$ concentrates. Eightyeight percent were commercial products, $9 \%$ homemade. Doses ranged 2-409 $\mathrm{mg}$.

Of children $(<19$ years; $n=15)$, most ingested edibles $(14 / 15,93 \%)$; gummies (5), candies (3), chocolates (3), cookies (2), and candied "orange slices"(1), with doses ranging $8-50 \mathrm{mg}$. Children who ingested $\leq 15 \mathrm{mg}$ were not less likely than those who ingested $>$ $15 \mathrm{mg}$ to be admitted to the hospital ( $0 / 3$ vs. $2 / 12, p=\mathrm{NS})$. Of adults ( $\geq 19$ years; $n=19)$, most ingestions were of edibles $(14 / 19,74 \%)$; brownies (3), caramels (3), gummies (2), candy (2), chocolate (2), cookie (1), and truffle (1). Fewer adults ingested concentrates (5/19,26\%); Rick Simpson Oil (1), Dab (1), and oil (3). One adult ingested $500 \mathrm{mg}$ resin and was admitted. Adults who ingested < $15 \mathrm{mg}$ or $<50 \mathrm{mg}$ were not less likely to be admitted (0/4 vs. $1 / 15$, $p=\mathrm{NS} ; 0 / 11$ vs. $1 / 8, p=\mathrm{NS}$ ).

Conclusion: While no THC-dose demonstrated a significantly different rate of admission to the hospital, no children who ingested < $15 \mathrm{mg}$ or adult who ingested $<50 \mathrm{mg}$ were admitted to the ward or ICU. Further studies may evaluate these pilot cutoffs as predictors of severity.

\section{Exposure to Antineoplastic Agents Reported to US Poison Control Centers, 2000-2017}

Joann Schulte ${ }^{1}$, Ashley Haynes ${ }^{2}$, Brandon Shank ${ }^{3}$, Kurt Kleinschmidt ${ }^{2}$, Brett Roth ${ }^{2}$, Anelle Menendez ${ }^{4}$, Eric Smith ${ }^{4}$

${ }^{1}$ Houston Health Department, Houston, Texas, USA. ${ }^{2}$ University of Texas Southwestern, Dallas, Texas, USA. ${ }^{3}$ MD Anderson, Houston, Texas, USA. ${ }^{4}$ Parkland Health and Hospital Systems, Dallas, Texas, USA

Purpose: Cancer patients are increasingly treated at home, and oncology treatment has expanded beyond the traditional cytotoxic agents to include targeted therapy (molecular and immunological) and endocrine therapy. Hypothesis: Residential exposures to antineoplastic agents are increasing over time.

Methods: We obtained retrospective surveillance data from the National Poison Data System (NPDS) of single exposures to all neoplastic agents during calendar years (CY) 2000-2017. Our goals were to describe the epidemiology of exposures in homes, what drugs were reported, demographics of exposed persons, and medical outcomes. The IRB approved this study.

Results: Callers reported 20,345 exposures to antineoplastic drugs; $86 \%(17,569)$ occurred in residential settings. Residential exposures increased 193\% between CY 2000 (475) and CY 2017 (1394). Only $4 \%$ (912) of exposures occurred in health care facilities and remained stable during the 18-year period. Among all exposures, most involved traditional cytotoxic agents $(13,287 ; 65 \%)$, followed by endocrine therapy $(4448 ; 22 \%)$ and targeted therapy (2535; $12 \%)$. Exposures were concentrated in children $<5$ years $(6695$; $33 \%)$ and persons aged 60 years or more $(6309 ; 31 \%)$. Fifty-one percent of exposures were therapeutic errors $(10,354 ; 51 \%)$, most with cytotoxic drugs $(6109 ; 58 \%)$, followed by endocrine therapy $(2959 ; 29 \%)$. Oral ingestion was the most common exposure route $(15,451 ; 76 \%)$. Nineteen percent of the exposures in children $<5$ (1295) involved aromatase inhibitors used in metastatic breast cancer. Forty deaths were reported; most among persons $\geq 60(25 ; 63 \%)$ and occurred at home $(29 ; 72 \%)$. All but one death involved cytotoxic drugs.
Conclusions: Most exposures to antineoplastic agents occur in homes and are increasing. The peak ages in reported exposures are among young children and older persons. Half the exposures are therapeutic errors. A better understanding of risks associated with exposures is needed.

007. Impact of a Hydromorphone Reduction Initiative on Opioid Pain Management Practices in the Emergency Department

Aaron Hettinger ${ }^{1}$, Daniel Hoffman ${ }^{2}$, Maryann Mazer-Amirshahi ${ }^{1}$, Jeffrey Dubin $^{3}$, Bonnie Levin ${ }^{3}$, Cynthia Webb ${ }^{3}$, William Frohna ${ }^{3}$

${ }^{1}$ MedStar Washington Hospital Center, Washington, DC, USA.

${ }^{2}$ MedStar Health, Washington, DC, USA. ${ }^{3}$ MedStar Health, Washington, DC, USA

Background: Hydromorphone is a potent opioid analgesic that has significant abuse potential and high rates of adverse drug events compared to other opioids. Despite these concerns, parenteral hydromorphone is commonly administered in many emergency departments (EDs).

Research Question: To determine the impact of a hydromorphone reduction initiative on opioid pain management practices in the ED.

Methods: This is a retrospective study comparing opioid administration in EDs across a nonprofit health system after opioid safety training and removal of parenteral hydromorphone from automated medication dispensing machines in five EDs. Patterns of opioid administration were analyzed in these EDs before and after the initiative took place.

Results: During the pre-intervention phase, an opioid analgesic was administered in $15.8 \%$ of visits vs. $14.2 \%$ post intervention, $(P<0.05)$. Parenteral (intravenous/intramuscular) opioids accounted for $65.3 \%$ of opioids administered pre-intervention and $62.7 \%$ postintervention (NS). In the pre-intervention phase, parenteral hydromorphone accounted for $49.3 \%$ of opioids administered in the ED. Morphine, oxycodone, hydrocodone, fentanyl, and codeine accounted for $31.6 \%, 23.7 \%, 5.1 \%, 1.6 \%$, and $1 \%$ of opioids administered respectively. After the intervention, parenteral hydromorphone accounted for $2.1 \%$ of opioids administered $(P<0.05)$. Morphine, oxycodone, and fentanyl utilization increased to $54.2 \%, 27.0 \%$, and $7.4 \%$ respectively $(P<0.05)$. Hydrocodone $(4.6 \%)$ and codeine $(1.2 \%)$ use remained stable (NS). The mean oral morphine milligram equivalents (MME) per dose pre-intervention was $12.9 \mathrm{mg}$ vs. $8.4 \mathrm{mg}$ post-intervention, a decrease of $35 \%$ $(P<0.05)$. Prior to the intervention, $25.8 \%$ of patients received two or more doses of opioid during their visit. After the intervention, $25.7 \%$ received two or more doses (NS).

Conclusion: The hydromorphone reduction initiative resulted in a dramatic decrease in parenteral use of this high-risk medication, whereas administration of several other opioids increased. There was an overall decrease in the oral MMEs per dose. Future research should examine patient outcomes related to such initiatives.

\section{Clinical Course and Management of the Heroin Body Stuffer}

Adrienne Hughes ${ }^{1}$, Lauren Murphy ${ }^{1}$, Tony Rianprakaisang ${ }^{1}$, Adam Blumenberg $^{2}$, Robert Hendrickson ${ }^{1,3}$

${ }^{1}$ Oregon Health and Science University, Portland, OR, USA. ${ }^{2}$ Oregon Health and Science University, Portland, OR, USA. ${ }^{3}$ Oregon Poison Center, Portland, OR, USA

Background: There is no consensus on the treatment or observation period for heroin body stuffers.

Objective: Describe the clinical course of heroin body stuffers to assist in determining their optimal management. 
Methods: Retrospective observational study. We searched the PC database for "heroin body stuffer" reported to a single institution between 1/1/ 2016 and 9/30/18. Data were extracted from hospital records. Inclusion: Ingestion of heroin that is (1) not for recreational purposes and (2) not body packing.

Results: Fifty-four cases met criteria: mean age 31 years (range 1967 years), 57\% men (31/54). Mean ingested mass was $2 \mathrm{~g}$ (range $0.1-$ $12 \mathrm{~g}, n=50 / 54$ ) in 1 packet (range $0-8$ packets). Median time to ED arrival was $2 \mathrm{~h}$ after ingestion $(n=52 / 54)$. The most common type of packet ingested (50/54 reported) was a well wrapped (sealed or tied off plastic baggie or balloon) $(19 / 50,38 \%)$, followed by loosely/poorly wrapped $(24 \%, 12 / 50)$, or unwrapped/loose drug in $12 \%(n=6 / 50)$. The mean length of observation was $23.6 \mathrm{~h}$. Only two $(3.7 \%)$ patients had recorded small pieces of plastic identified in their stool. Sixty-one percent (33/54) developed at least one symptom of opioid intoxication (somnolence/lethargy, 29; miosis, 2; hypoxia, 1) and 31/33 developed symptoms early $(<3 \mathrm{~h})$. Both patients with late opioid toxicity ingested large quantities ( $4 \mathrm{~g}$ and $3 \mathrm{~g}$ ). Five patients received naloxone in the prehospital setting and five in the ED in response to CNS depression $(9 / 10)$ and hypoxia $(1 / 10)$. The median time until resolution of symptoms was $10 \mathrm{~h}(n=26 / 33$, range $15 \mathrm{~min}$ to $33 \mathrm{~h})$. Twenty-eight percent $(15 / 54)$ received activated charcoal. Twenty percent $(n=11 / 55)$ received abdominal radiographs, and 3\% $(n=2 / 55)$ abdominal CT scans; none detected any packets.

Conclusion: These data indicate higher rates of opioid intoxication in heroin body stuffers. Delayed toxicity occurred in a small percentage of patients. A prospective study on heroin body stuffers may be more useful for determining the optimal management strategy for these patients.

\section{Characterization of Carfentanil Exposure in Patients Presenting to a Large Urban Healthcare System}

Arkady Rasin ${ }^{1,2}$, Neeraj Chhabra ${ }^{2}$, Lum Rizvanolli ${ }^{2}$, Juleigh Konchak ${ }^{2}$, Steven E Aks ${ }^{1,2}$

${ }^{1}$ Toxikon Consortium, Chicago, IL, USA. ${ }^{2}$ Cook County Health, Chicago, IL, USA

Background: Carfentanil is a high potency synthetic opioid detected in fatalities and police seizures of narcotics. However, exposure patterns in living patients are poorly characterized in the literature.

Research Question: Are patients presenting to a large urban healthcare system exposed to carfentanil through illicit drug use?

Methods: This is a retrospective analysis of all patients with a urine ELISA drugs of abuse screen ordered between May 23, 2018 and July 5, 2018 in a large urban healthcare system. Screens positive for opioids or fentanyl were reflexed to a designer opioid panel $(1480 \mathrm{u}$, NMS Labs). Charts of patients testing positive for carfentanil were abstracted for relevant clinical data.

Results: There were 142 designer opioid panels sent during the study period: 10 panels were positive for carfentanil in 7 patients. All patients identified as black and five were women. Median age was 51 years (range 39-63 years). Seven of ten carfentanil-positive samples were sent from the medication assisted therapy (MAT) clinic, one from the chronic pain clinic, and one from the emergency department (ED). Two patients from the MAT clinic had multiple positive tests for carfentanil, one on three occasions each 1 week apart, and one on two occasions 1 week apart. Two patients presented with symptoms consistent with opioid toxicity, one to the ED requiring $4 \mathrm{mg}$ naloxone, and one to the chronic pain clinic but did not require naloxone. All samples that tested positive for carfentanil also tested positive for multiple fentanyl analogs. Eight of ten samples also tested positive for 4-ANPP and 2-furanylfentanyl. None of the patients endorsed intravenous use of opioids. All MAT clinic patients were on buprenorphine therapy.
Conclusion: Carfentanil was identified in patients presenting to both the ED and outpatient settings, including in patients actively participating in MAT.

010. Patient Perceptions of Emergency Department Naloxone and Suboxone Distribution: Findings from a Single Center Naloxone Distribution Program

Jennifer Love, Rachel Graves, Frances Shofer, Jeanmarie Perrone Department of Emergency Medicine, University of Pennsylvania, Philadelphia, PA, USA

Background: In 2017, 1200+ unintentional overdose deaths occurred in Philadelphia; $88 \%$ were opioid-related deaths. Emergency department (ED) providers frequently encounter opioid use disorder (OUD) patients in crisis. Naloxone dispensing and buprenorphine (suboxone) initiation are real-time interventions for mitigating OUD risk.

Hypothesis: OUD patients are interested in obtaining intranasal naloxone and/or suboxone from ED providers. OUD patients with subsequent ED visits will receive prescriptions for naloxone.

Methods: A convenience sample was enrolled at an academic ED between 10/2016 and 11/2017. Adult patients who received naloxone by EMS or ED providers were taking medication-assisted treatment (MAT) and had a related complaint (i.e., respiratory depression), or displayed signs suggestive of opioid intoxication were eligible. Patients or family members completed a survey and received intranasal naloxone training and two Narcan ${ }^{\mathrm{TM}}$ nasal atomizers. We collected data on return ED visits for enrolled patients following their initial encounter until 10/2018.

Results: Twenty-five patients completed the survey study. Sixty-eight percent were male. Mean age was 43 years old. In the month prior to their ED visit, $76 \%$ had used heroin, and $28 \%$ had used prescription opioids. Patients noted that suboxone can help with withdrawal (48\%) and control cravings ( $56 \%$ ) but agreed that it can cause withdrawal symptoms $(68 \%)$. Seventy-eight percent of patients would be interested in receiving suboxone from the ED. Seventy-two percent of patients had received previous naloxone treatment. Patients overwhelmingly agreed that they would want naloxone if they overdosed $(96 \%)$. One hundred percent were interested in receiving take-away naloxone, and most $(96 \%)$ would tell a family member/friend that they had naloxone. Twenty-eight percent of patients returned to the ED during the follow-up period. One patient received one additional naloxone prescription on a return visit.

Conclusions: Most patients with OUD treated in an ED have prior naloxone and suboxone experience and were interested in receiving suboxone and naloxone from the ED.

\section{Day 1: Posters, Abstracts 011-054}

\section{Association of Ingested Dose and Severity of Toxicity in Heroin Body Stuffers}

Adrienne Hughes ${ }^{1}$, Lauren Murphy ${ }^{1}$, Adam Blumenberg ${ }^{1}$, Tony Rianprakaisang $^{2}$, Robert Hendrickson ${ }^{1,3}$

${ }^{1}$ Oregon Health and Science University, Portland, OR, USA. ${ }^{2}$ Oregon Health and Science University, Portland, OR, USA. ${ }^{3}$ Oregon Poison Center, Portland, OR, USA

Background: There are little data available regarding severity of toxicity, ingested dose, and duration of effects in the heroin body stuffer.

Objective: Compare the severity and duration of opiate toxicity in heroin body stuffers based on reported ingested dose.

Methods: Retrospective observational study. We searched the PC database for "heroin body stuffer" reported to a single institution between $1 / 1$ / 2016 and 9/30/18. Data were extracted from hospital records. Inclusion: ingestion of heroin that is (1) not for recreational purposes and (2) not body packing. 
Results: Fifty-four cases resulted: mean age 31 years (range 19-67 years), $57 \%$ men (31/54). Mean ingested mass was $2 \mathrm{~g}$ (range $0.1-12 \mathrm{~g}, n=50 /$ 54 ) in one packet (range $0-8$ ). We found no significant difference in the amount of heroin ingested in stuffers who developed symptoms of opiate intoxication ( $n=31 / 50$, mean $2.56 \mathrm{~g}$, median $2 \mathrm{~g}$, range $0.4-12 \mathrm{~g}$ ) compared to stuffers who developed no evidence of opiate intoxication $(n=$ $19 / 50$, mean $2.48 \mathrm{~g}$, median $2.5 \mathrm{~g}$, range $0.1-7 \mathrm{~g}$ ). The median time until resolution of symptoms was $10 \mathrm{~h}(n=26 / 33$, range $15 \mathrm{~min}$ to $33 \mathrm{~h})$. Time until resolution of symptoms of opiate intoxication in those patients who ingested $>1.5 \mathrm{~g}(n=13 / 24$, range $1.75-4 \mathrm{~g}$, mean $12.8 \mathrm{~h}$, median $12 \mathrm{~h}$, range $2-33 \mathrm{~h}$ ) was not significantly different than time to resolution of symptoms in those patients who ingested $<1.5 \mathrm{~g}(n=11 / 24$, range 0.4 $1.5 \mathrm{~g}$, mean $8.7 \mathrm{~h}$, median $6 \mathrm{~h}$, range $15 \mathrm{~min}-24 \mathrm{~h}$ ). We found no significant difference in the amount of heroin ingested in those patients who received naloxone ( $n=8 / 50$, mean $2.36 \mathrm{~g}$, median $2.5 \mathrm{~g}$, range $0.4-4 \mathrm{~g})$ compared to patients who did not receive naloxone $(n=42 / 50$, mean $2.56 \mathrm{~g}$, median $2 \mathrm{~g}$, range $0.1-12 \mathrm{~g}$ ).

Conclusion: Symptoms of opiate intoxication, duration of toxicity, and use of naloxone do not appear to be related to the amount of drug ingested in heroin body stuffers.

012. Characteristics of Posts of Opioid Users on Reddit, an Online Social Media Forum, an Area for Improved Harm Reduction

Daniel Overbeek ${ }^{1}$, Alexander Janke ${ }^{2}$

${ }^{1}$ University of Michigan, Ann Arbor, MI, USA. ${ }^{2}$ Yale University, New Haven, CT, USA

Background: We wished to characterize postings made on the Reddit.com forum "Opiates." In particular, we tested the hypothesis that the proportion of online posts glorifying opioid use is greater than the proportion advocating harm reduction.

Methods: This is an analysis of posting to the Reddit.com forum "Opiates" (reddit.com/r/opiates). The posts were then categorized by their main topic, including "glorifying use, including obtaining and consuming," "harm reduction," etc. We identified specific characteristics of posts, such as those which endorse unsafe behavior (beyond opioid use). Results: Five hundred posts were obtained; 34 were excluded as duplicates or unanalyzable and 466 were analyzed. The proportion "glorifying use" was $43.9 \%$, and the proportion "advocating harm reduction" was $15.4 \%$. Difference in proportion was $+28.5 \%(95 \% \mathrm{CI}+22.7 \%$ to + $34.3 \%)$. Ten posts $(2.1 \%)$ discussed avoiding law enforcement, and 13 posts $(2.8 \%)$ discussed interacting with physicians, including how to obtain stronger prescriptions. Within the above topics, $19.3 \%$ of posts endorsed unsafe behavior, beyond use for simple analgesia. These included many posts endorsing polysubstance use, as well as one unique post encouraging patients to follow reports of deadly fentanyl outbreaks to obtain more potent opioids. Only $15 \%$ of posts encouraged harm reduction, including encouragement and treating withdrawal symptoms. The consumption of pharmaceutical opioids appeared very commonly, discussed in $47.6 \%$ of the posts.

Conclusion: Online forums such as the Reddit.com forum "Opiates" advertise themselves as harm reduction forums, but our data show that these forums instead have a small amount of harm reduction information. Many posts glorify unsafe drug use. Sites like these may represent an impediment to educating patients with opiate use disorder on safe use and effective treatment.

\section{Recreational Use of Kratom (Mitragynine) as Discussed in an Online Drug Forum}

Daniel Overbeek ${ }^{1}$, Alexander Janke ${ }^{2}$

${ }^{1}$ University of Michigan, Ann Arbor, MI, USA. ${ }^{2}$ Yale University, New Haven, CT, USA

Background: The use of kratom (mitragynine) as an alternate opioid has been escalating in parallel with the opioid epidemic, and it is often called a "natural and legal high." We wished to analyze how users of kratom were discussing its use on online drug forums.

Methods: This is a retrospective analysis of posts made to the Reddit.com "Opiates" forum. We used a data extraction interface to extract 100 consecutive posts on the "Opiates" forum with the keyword "kratom." The posts were then characterized regarding multiple possible characteristics, including the main topic of the post. We also identified kratom was being used for withdrawal symptoms, in combination with other opioids, and if users were combining kratom use with other opioid use. We also identified which posts discussed the safety of kratom use.

Results: A total of 100 posts were analyzed. Most posts (57\%) were discussing how to use or obtain kratom. There was also a small number of posts $(4 \%)$ discussing the legal implications of kratom use, including whether it could be detected in standard legal drug testing, such as those used for individuals on probation. Forty-two percent of posts discussed using kratom to treat opioid withdrawal symptoms. Three percent of posts discussed possible dangers of kratom. Additionally, $52 \%$ of the posts discussed combining kratom with other illicit or pharmaceutical opioids.

Discussion: Reddit is a well-trafficked drug forum, with significant discussions regarding kratom use. Most posts expounded the idea that kratom can be safely used, including one with this statement: "Dude kratom is harmless, a much better alternative than heroin." Users are also combining kratom with other opioids, with possibly dangerous consequences. Providers need to be aware of these trends and should improve patient education regarding possible dangers of these "natural products."

014. The Impact of the Parenteral Opioid Medication Shortage on Opioid Utilization Practices in the Emergency Department

Amanda Deutsch ${ }^{1}$, David Gajdosik ${ }^{1}$, Lyudmila Garbosky ${ }^{1}$, Maryann Mazer-Amirshahi ${ }^{2}$, Erin Fox ${ }^{3}$, Jeanmarie Perrone ${ }^{1}$

${ }^{1}$ University of Pennsylvania, Philadelphia, Pennsylvania, USA. ${ }^{2}$ MedStar Washington Hospital Center, Washington, DC, USA. ${ }^{3}$ University of Utah, Salt Lake City, Utah, USA

Background: Pain is the most common reason that patients visit the Emergency Department (ED). Due to the escalating opioid crisis, multiple guidelines have focused on judicious opioid use. However, the recent parenteral opioid medication shortage generated an alternative rationale to limit intravenous (IV) opioid use.

Hypothesis: We hypothesized that during months of opioid shortages, emergency providers utilize less IV opioids, shift a proportion of IV to oral use, and that medication waste increased due to repackaging.

Methods: This is a retrospective review of opioid medication utilization in the ED of an urban referral hospital with 65,000 annual visits. We compared opioid utilization and waste during 2 months of opioid shortages and their respective control months 1 year prior. We evaluated the means between control (2017) and shortage (2018) months with twotailed $t$ tests.

Results: The number of ED patient visits were similar in control vs. shortage months: 5171 vs. 5180 March 2017 vs. 2018; 5327 vs. 5246 May 2017 vs. 2018, $p=$ NS. During opioid shortage months, fewer ED patients received IV opioids: 550 vs. 380 March 2017 vs. 2018; 544 vs. 398 May 2017 vs. 2018, $p=0.02$. Patients who received oral opioids: 346 vs. 315 March 2017 vs. 2018 , 359 vs. 311 May 2017 vs. 2018, $p=$ NS. Total morphine milligram equivalents per patients receiving opioids: 91 mg vs. 99 mg March 2017 vs. 2018, 88 mg vs. 72 mg May 2017 vs. $2018, p=$ NS. Total opioid waste in oral morphine equivalents: $51,552 \mathrm{mg}$ vs. $66,006 \mathrm{mg}$ March 2017 vs. 2018 , and $80,578 \mathrm{mg}$ vs. $35,256 \mathrm{mg}$ May 2017 vs. 2018, $p=\mathrm{NS}$.

Conclusions: Opioid utilization decreased overall during opioid shortage months. However, the hypothesized shift of parenteral to oral opioid use during opioid shortage months was inconsistent. Opioid use per patient did not change. 
015. Utilization and Results of an Expanded Urinary Opioid Screen Including Fentanyl in a Healthcare System

Neeraj Chhabra ${ }^{1,2}$, Arkady Rasin ${ }^{1,2}$, Lum Rizvanolli ${ }^{1}$, Gisela Bryan ${ }^{1}$, Caleb Scarth ${ }^{1}$, Steven E Aks ${ }^{1,2}$

${ }^{1}$ Cook County Health, Chicago, IL, USA. ${ }^{2}$ Toxikon Consortium, Chicago, IL, USA

Background: Urinary immunoassay antibody testing for the detection of opiates has been widely utilized for workplace screening. Such testing has since migrated to the clinical setting despite its limitation to the detection of morphine and substances with structurally similar metabolites that may cross-react with the immunoassay. The test is thus prone to false-negative results, limiting the detection of synthetic opioids. In May of 2018, an expanded urinary opioid screening test-adding the capability of detecting fentanyl, hydrocodone, and oxycodone to methadone-was introduced to supplement the standard urinary opiate screen at a large urban public healthcare system.

Research Question: How is an expanded urinary opioid screening test being utilized by a healthcare system and how frequently are fentanyl and other synthetic opioids being detected?

Methods: This is a retrospective analysis of expanded urinary opioid screening tests performed from May through July 2018. Screening tests were conducted by antibody immunoassay using a Beckman Analyzer (AU5800) with Thermo-Fisher reagents. Treating clinicians ordered urine drug testing at their discretion.

Results: A total of 2099 expanded urinary opioid screens were conducted. Thirty-one percent of screens were sent from the outpatient setting, 28\% from the Emergency Department/Trauma setting, 20\% from the inpatient setting, $18 \%$ from the correctional health system, and 3\% from other locations. Overall rates of opioid detection were as follows: opiates, $16.0 \%$; methadone, $18.0 \%$; fentanyl, $15.6 \%$; hydrocodone, $6.7 \%$; and oxycodone, $0.5 \%$.

Conclusions: The expanded urinary opioid screen was heavily utilized throughout the healthcare system. In addition to opiates, substantial numbers of fentanyl were detected in addition to methadone, hydrocodone, and oxycodone. Further research is necessary to determine what proportion was illicit in nature, either knowingly or as a contaminant. Such data can be utilized to identify high-risk patients for overdose death who may benefit most from targeted interventions.

\section{Malignant Cerebellar Edema Following Fentanyl Patch Exposure in an Infant}

Louise Kao, Lindsey Haut, Rupa Radhakrishnan, Riad Lutfi, Laurie Ackerman

Indiana University, Indianapolis, IN, USA

Background: Unintended consequences of the opioid epidemic include increased exposure in children. Pediatric opioid-induced neurotoxicity is increasingly reported.

Case Report: An 8-month-old previously healthy child presented with lethargy. On ED presentation, vital signs were T $36.2{ }^{\circ} \mathrm{C}$; HR 134/min; RR 28/min; BP 96/56; $\mathrm{O}_{2} 77 \%$. Physical examination was remarkable for extreme drowsiness and decreased motor tone. Rapid glucose was $43 \mathrm{mg}$ / dL. Head CT was read as extensive symmetric areas of low attenuation in the cerebellum, suggestive of infarcts (image available). Patient was given dextrose, fluids, and ceftriaxone. On arrival to our hospital, glucose was $30 \mathrm{mg} / \mathrm{dL}$ and he had increasing lethargy. Upon intubation, a $25 \mu \mathrm{g} / \mathrm{h}$ fentanyl patch was removed from the soft palate. At that time, a family member noted the missing patch. Toxicology was consulted, and the diagnosis of malignant cerebellar edema was made. Due to declining neurologic status and worsening findings on imaging, patient underwent suboccipital craniectomy and placement of an external ventricular drain on Hospital Day (HD) \#2. He was extubated HD \#4 and discharged to the ward $\mathrm{HD \# 7}$ with a reassuring neurological exam. A ventriculoperitoneal shunt was placed HD \#10, and he was discharged to foster home on HD \#28 with favorable neurologic status. Serum fentanyl level sent HD\#1 was $12 \mathrm{ng} / \mathrm{mL}$, and norfentanyl level was $100 \mathrm{ng} / \mathrm{mL}$. Therapeutic levels are between 1 and $4 \mathrm{ng} / \mathrm{mL}$.

Discussion: Malignant cerebellar edema is described rarely following pediatric opioid intoxication, and likely represents a distinct entity of pediatric opioid-induced neurotoxicity. The mechanism may be related to opioid receptor-mediated changes in neuronal metabolism exacerbated by hypoxic insult, to which pediatric patients are uniquely susceptible. In our case, prompt diagnosis and neurosurgical intervention resulted in a favorable outcome. Clinicians should be aware of this rare but deadly syndrome.

017. Methadone-Associated QT Prolongation and Polymorphic Ventricular Tachycardia: an Inpatient Transition to Buprenorphine

Loren Touma ${ }^{1}$, Vince Desiato ${ }^{1}$, Sarah Weiss ${ }^{2}$, Steven Walsh ${ }^{2}$, Serge Simpson ${ }^{2}$

${ }^{1}$ William Carey University College of Osteopathic Medicine, Hattiesburg, MS, USA. ${ }^{2}$ Einstein Medical Center Philadelphia, Philadelphia, PA, USA

Background: Methadone is used for opioid addiction but is associated with QTc prolongation and polymorphic ventricular tachycardia (PVT). Discontinuation of methadone induces protracted withdrawal symptoms, but the long half-life of methadone and partial antagonist properties of buprenorphine may precipitate withdrawal if buprenorphine is started too early.

Hypothesis: A patient with methadone-induced QTc prolongation and PVT could transition from methadone to buprenorphine in-hospital with minimal withdrawal symptoms using short-acting replacement opioids.

Methods: A 65-year-old man with history of hepatitis $\mathrm{C}$, diastolic heart failure, hypertension, and heroin abuse treated with methadone for 10 years presented with three days of frequent syncopal episodes lasting 1 to 2 min associated with dizziness and chest pressure. On arrival, his heart rate was $48 \mathrm{bpm}$. He suffered a monitored episode of PVT; EKG at that time displayed a QT/QTc of $666 \mathrm{~ms} / 551 \mathrm{~ms}$. His EKG from before starting methadone had a normal QT/QTc. He was admitted to the cardiac intensive care unit where methadone was held and he experienced frequent PVT for the first 2 days. He improved on day 3 but began to experience withdrawal, so half-dose methadone was given with symptomatic improvement but recurrence of PVT. Medical toxicology was consulted, and we transitioned therapy using oxycodone $20 \mathrm{mg}$ every $3 \mathrm{~h}$ for the next 3 days with adjunctive as-needed hydromorphone, lorazepam, melatonin, and ibuprofen. On the fourth day, he was given no opioids for $12 \mathrm{~h}$, and then started buprenorphine therapy.

Results: The patient responded well and was successfully transitioned to buprenorphine. His cardiac abnormalities completely resolved, returning to his pre-methadone baseline. He transitioned to outpatient addiction treatment by warm handoff.

Conclusion: Methadone to buprenorphine transition can be performed in-hospital with short-acting opioid replacement therapy in appropriate cases.

\section{Can Reddit Reveal Unique Facets of Suboxone Use and Misuse?}

Rachel Graves ${ }^{1}$, Jeanmarie Perrone ${ }^{1}$, Jennifer Love ${ }^{1}$, Abeed Sarker ${ }^{2}$ ${ }^{1}$ Department of Emergency Medicine, University of Pennsylvania, Philadelphia, PA, USA. ${ }^{2}$ Department of Biostatistics, Epidemiology and Informatics, Perelman School of Medicine, University of Pennsylvania, Philadelphia, PA, USA

Background: Buprenorphine-naloxone "Suboxone" prescribing in the USA has expanded in an attempt to combat the rising deaths from the opioid crisis. Ethnographic studies of patients who use Suboxone illicitly but for therapeutic endpoints can provide limited information. We 
proposed that Reddit may provide surrogate observations of Suboxone use in this community.

Research Question: Can Reddit serve as a resource to study the behaviors and perceptions about suboxone in people with opioid use disorder? Methods: This is a mixed methods observational study of posts from the Reddit social network - which is a unique medium that enables users to post anonymously in topic-specific forums, known as subreddits. We collected data from the r/suboxone subreddit for approximately 3 months-May 23 to August 27, 2018 via Google BigQuery. Two clinicians manually analyzed a sample of posts from ten threads within the subreddit using the grounded theory approach — posts were first categorized into fine-grained topics and then these were merged into five coarsegrained themes (suggestive, personal experience, informational, information seeking, and other). Two hundred subreddit posts were analyzed in this manner, and the distributions of the themes were quantitatively compared, and the topics were qualitatively analyzed.

Results: Among the 200 posts, there were 83 (41.5\%) suggestive, 30 (15\%) informational, $79(39.5 \%)$ personal experience, 21 (10.5\%) information seeking, and $18(9 \%)$ other posts, including posts that contained multiple themes. The posts covered a variety of topics, the most common being "using suboxone for medication assisted treatment (MAT) from other opioids," "correct way of using suboxone," "suboxone withdrawal symptoms," "tapering off suboxone," and "suboxone abuse with other substances."

Conclusion: Reddit can serve as a unique source of information about suboxone, especially about tapering strategies and misuse that may not be available in more formal investigations.

\section{Sentiment, Themes, and Analyses in Tweets about Suboxone}

Jennifer Love ${ }^{1}$, Jeanmarie Perrone ${ }^{1}$, Rachel Graves ${ }^{1}$, Abeed Sarker ${ }^{2}$ ${ }^{1}$ Department of Emergency Medicine, University of Pennsylvania, Philadelphia, PA, USA. ${ }^{2}$ Department of Biostatistics, Epidemiology and Informatics, Perelman School of Medicine, University of Pennsylvania, Philadelphia, PA, USA

Background: As the opioid crisis escalates, buprenorphine-naloxone (Suboxone) therapy is emerging as a leading opioid use disorder (OUD) treatment. It has a large diversion presence and is utilized illicitly to prevent or treat opioid withdrawal. Little is known about public perceptions of Suboxone including illicit therapeutic use or misuse. We sought to characterize Twitter posts to assess the sentiment and themes mentioning Suboxone. A better understanding of Suboxone may help inform patient engagement in therapeutic opportunities.

Research Question: Can Twitter serve as a resource to study behaviors and perceptions about Suboxone to expand clinicians' knowledge about Suboxone's therapeutic role?

Methods: A mixed-methods observational study of social media posts mentioning Suboxone was performed. We collected posts mentioning "Suboxone" from Twitter via its public API. We manually categorized a random tweet sample and grouped categories into four themes: personal consumption, misuse/abuse, information, and unrelated. User sentiments expressed in the tweets were coded as negative, positive, neutral, and unclear. We compared category and sentiment distributions.

Results: Among 300 tweets, 110 (36.7\%) were about personal consumption, $121(40.3 \%)$ were informational, $24(8.0 \%)$ indicated misuse/abuse, and $45(15 \%)$ were unrelated or difficult to categorize. Tweets belonging to the information and consumption themes commonly expressed information about effectiveness of the drug and adverse effects (e.g., withdrawal symptoms). Misuse/abuse tweets revealed information about Suboxone co-ingestion with other substances (e.g., alcohol). Sentiment sub-analysis revealed that 46 (15.3\%) expressed positive, 57 (19.0\%) negative, and $152(50.7 \%)$ expressed neutral sentiment. Chi-squared testing revealed no difference in negative and positive sentiment distribution for tweets $(p=0.28)$. Negative sentiment tweets often commented on ineffectiveness, stigma and skepticism; positive sentiment tweets commented on effectiveness and misuse.
Conclusion: Twitter data can provide insights about Suboxone and inform the therapeutic community about barriers and perceptions regarding Suboxone use. Further studies are warranted.

020. A 3-Day Phenobarbital Protocol for Benzodiazepine Detoxification-Description of Use During Buprenorphine Initiation in a Detoxification Facility

Timothy Wiegand

Department of Emergency Medicine at the University of Rochester Medical Center, Rochester, New York, USA

Background: Patients with opioid use disorder often have other substance use disorders, which providers need to be aware of to effectively treat. Benzodiazepine dependence is one of the most common of these occurring in $20-30 \%$ of patients receiving buprenorphine treatment. Benzodiazepine dependence itself is associated with a variety of adverse outcomes, and concomitant use increases risk of adverse outcomes associated with buprenorphine maintenance. Helping patients wean from benzodiazepines is challenging. While a prolonged and gradual taper is typically advised, successful completion of this occurs infrequently as patients often return to regular use before completion. We describe an alternative treatment using a 3-day phenobarbital protocol.

Hypothesis: A 3-day phenobarbital protocol will be successful for detoxification from benzodiazepines for patients initiating buprenorphine. Methods: Retrospective review of three patients' charts.

Cases: Three benzodiazepine- and opioid-dependent (heroin/fentanyl) patients (two male, one female, age 22-36) were treated; two were prescribed benzodiazepines, one reported only illicit. Daily doses ranged 4 $8 \mathrm{mg}$ alprazolam (2) and 4-12 mg clonazepam (1). Two patients abused gabapentin; none reported alcohol. All three reported withdrawal symptoms with benzodiazepine cessation but had no symptoms at protocol start. All received a test dose of $130 \mathrm{mg}$ phenobarbital orally followed by $130 \mathrm{mg}$ every $4 \mathrm{~h} \times 6,130 \mathrm{mg}$ every $6 \mathrm{~h} \times 4$, and $130 \mathrm{mg}$ every $8 \mathrm{~h} \times 3$. Nobody needed doses held for somnolence. All patients underwent standard buprenorphine initiation protocol transitioning to maintenance. All three were benzodiazepine free at follow-up 2 weeks later.

Discussion: Phenobarbital is a long-acting lipid soluble barbiturate. After appropriate dosing, it redistributes from lipid stores providing a slow "auto-taper." Pairing benzodiazepine detoxification to buprenorphine maintenance may be particularly effective as patients are motivated to continue buprenorphine.

Conclusions: A 3-day phenobarbital protocol allowed for successful detoxification in three benzodiazepine-dependent patients during buprenorphine initiation.

021. Optimal Dose and Frequency with Multimodal AnalgesiaCase Study of Acute/Perioperative Pain Management during Buprenorphine Treatment

Bryan Ross, Nicholas Nacca, Timothy Wiegand

Department of Emergency Medicine at the University of Rochester Medical Center, Rochester, New York, USA

Background: Buprenorphine is a high affinity, long-acting, partial mu receptor agonist with kappa antagonist effects. While buprenorphine is most commonly associated with the treatment of opioid dependence, it was first available as an IV formulation for the treatment of moderate to severe pain. In recent years, additional formulations have been approved for the treatment of pain including a transdermal patch along with lower-dose SL formulations. We present a patient receiving buprenorphine/naloxone (BNX) for opioid use disorder who underwent an above-the-knee amputation and describe his peri- and postoperative pain management.

Hypothesis: Effective analgesic control can be achieved for patients receiving buprenorphine maintenance when dose and frequency are optimal and multimodal pain control is used. 
Methods: Retrospective chart review single patient

Case: A 62-year-old male with history of IV heroin abuse and total knee arthroplasty was admitted for an urgent above-the-knee amputation. $\mathrm{He}$ was taking 8-2 mg BNX films SL BID as an outpatient. Pre-operatively, 4-1 mg SL QID was used with scheduled NSAID and acetaminophen doses. Peri-and postoperatively, epidural bupivacaine was initiated, and BNX increased to 8-2 mg QID with good pain control. Pain returned as the epidural was weaned off, and IV buprenorphine $300 \mu \mathrm{g}$ every $4 \mathrm{~h}$ PRN was added. Good pain control returned. The patient was ultimately discharged on 8-2 mg BNX films QID. At follow-up 4 weeks later, he had excellent pain control and he gradually returned to original BNX dosing.

Discussion: Optimal buprenorphine dosing for analgesia is more frequent than for dependence. Doses should be titrated up after appropriate timing, and IV buprenorphine can be utilized for breakthrough pain. Multimodal pain control, such as epidural anesthesia, and non-opioid analgesics, will help maximize analgesia.

Conclusions: Appropriate dosing of buprenorphine and multimodal analgesia should be leveraged for the treatment of acute/perioperative pain control in patients receiving buprenorphine for opioid dependence.

\section{Single Agent Bupropion Overdose: Effects as Reported in the} ToxIC Registry

Brian Murray, Joseph Carpenter, Camille Dunkley, Tim Moran, Waleed Alsukaiti, Musa S Alfaifi, Ziad Kazzi

Emory University School of Medicine, Atlanta, GA, USA

Introduction: Bupropion, a monocyclic phenylethylamine antidepressant, is the only FDA-approved synthetic cathinone. It increases the release of norepinephrine in the locus coeruleus and dorsal raphe which leads to an increase in the frequency of serotonergic neuron firing. The diagnosis of serotonin syndrome (SS) from bupropion poisoning is controversial due to the lack of direct serotonergic activity. Nonetheless, there is one documented report of SS after single agent bupropion overdose.

Methods: This is a qualitative analysis of the clinical effects from singleagent bupropion overdose and characteristics associated with patients who developed SS versus the ones who did not. The data were obtained from the Toxicology Investigators Consortium (ToxIC) registry between $01 / 01 / 2014$ and 12/31/2017. Categorical variables were described as percentages with $95 \%$ confidence intervals. Odds ratios and $95 \%$ confidence intervals were computed using logistic regressions for binary variables and an ordinal logistic regression for Hunter's criteria.

Results: There were 266 single-agent bupropion ingestions recorded; of these, $170(63.9 \%)$ were female and 179 (67.3\%) Caucasian. The most common symptoms were seizures $(n=104,39.1 \%)$, agitation $(n=72,27.1 \%)$, toxic psychosis $(n=45,16.9 \%)$, and myoclonus/tremor/hyperreflexia $(n=42,15.8 \%)$. Benzodiazepines were the most common therapy $(n=159,59.8 \%)$. There were 13 $(4.9 \%, 95 \% \mathrm{CI}=2.8-8.3 \%)$ patients diagnosed with $\mathrm{SS}$ as listed in the registry by the medical toxicology physician who evaluated the patient at the bedside. Age, race, ethnicity, and gender were not associated with the diagnosis of SS, nor were seizures, agitation, or toxic psychosis. The only symptom associated with the diagnosis of SS was myoclonus/tremor/hyperreflexia $(\mathrm{OR}=10.52,95 \% \mathrm{CI}=$ 3.25-34.1).

Conclusion: Bupropion is associated with seizures, agitation, toxic psychosis, and myoclonus/tremor/hyperreflexia. It is the sole cause of SS in $5 \%$ of patients reported in ToxIC. The only characteristic associated with the diagnosis of SS was myoclonus/tremor/ hyperreflexia.

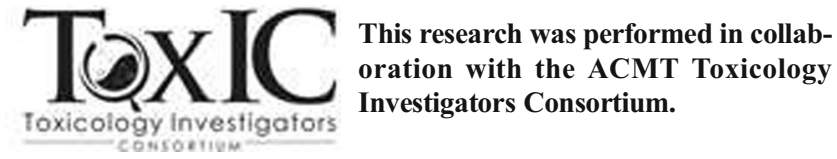

023. Case Series of Bupropion Dosing Errors with Major Effects Reported to the California Poison Control System

Matthew Riddle, Alicia Minns, F Lee Cantrell

UCSD, San Diego, CA, USA

Background: Bupropion is a commonly prescribed antidepressant, smoking-cessation aid, and weight loss agent. There is a wellrecognized risk of seizures with therapeutic dosing, and seizures occur in about $20-30 \%$ of acute overdoses. Significant cardiac effects and shock from bupropion toxicity are rare but potentially life-threatening.

Research Question: Determine the frequency of major effects of bupropion dosing errors and characterize the toxic effects of bupropion in this patient group.

Methods: This is a retrospective patient case series. We performed a retrospective review of all therapeutic bupropion dosing errors with major effects reported to the California Poison Control System (CPCS) over a 15-year period between 2003 and 2017.

Results: During this period, there were 1404 total cases of therapeutic bupropion dosing errors reported to the CPCS. There were 14 cases (1\%) with major effects. Of these cases, $71 \%$ were female, and the median age was 34 years old. The median dose of bupropion was $600 \mathrm{mg}$. The most common effects seen were tachycardia (64\%), seizure (57\%), confusion/ agitation (43\%), and tremor (29\%). A simple double dose of the patient's prescribed bupropion was the exposure source in $8(57 \%)$ of these 14 cases. There were no cases of status epilepticus or significant cardiac toxicity and no deaths.

Conclusion: Overall, there was a low frequency of major effects seen in bupropion dosing errors; however, even a single double dose of bupropion may lead to clinically significant toxicity.

\section{Two Cases of Bupropion-Related Cardiogenic Shock Treated} with Early Institution of Extracorporeal Membrane Oxygenation

Daniel Ovakim ${ }^{1,2}$, Meredith Briggs ${ }^{3}$, Kelly Phillips ${ }^{1}$, Lynn Fedoruk ${ }^{1}$ ${ }^{1}$ Vancouver Island Health Authority, Victoria, British Columbia, Canada. ${ }^{2} \mathrm{BC}$ Drug and Poison Information Centre, Vancouver, British Columbia, Canada. ${ }^{3}$ University of British Columbia, Victoria, British Columbia, Canada

Background: Bupropion is a commonly prescribed psychoactive medication used for the treatment of depression and other psychiatric disorders. Bupropion overdose presents with sympathomimetic features and delayed neuro- and cardiac toxicity often refractory to standard treatments. Extracorporeal membrane oxygenation (ECMO) has been used as a rescue therapy for severe cardiovascular collapse.

Methods: This report is a review of two cases. In the first case, a 17-yearold female with a history of depression ingested an unknown amount of bupropion XL, escitalopram, and quetiapine. She presented with a mild sympathomimetic toxidrome, however developed recurrent seizures and a wide complex dysrhythmia associated with cardiogenic shock $36 \mathrm{~h}$ after ingestion. In anticipation of further deterioration, she was placed on venoarterial ECMO (VA-ECMO). The second case is a 23-year-old male that ingested $15 \mathrm{~g}$ of bupropion XL. Although initially stable, he required intubation due to declining level of consciousness. ECG demonstrated a QRS of $114 \mathrm{~ms}$, which persisted despite several ampules of sodium bicarbonate. Due to escalating vasopressor requirements and persistent QRS-widening on ECG he was placed on VA-ECMO.

Results: In the first case, following improvement in ECG and echocardiographic features, the patient underwent successful decannulation after $72 \mathrm{~h}$ and recovered without sequelae. The second patient was decannulated after $48 \mathrm{~h}$ and recovered normally.

Discussion: We present two cases in which ECMO was employed early in the course of severe drug-induced cardiogenic shock involving bupropion. While we do not have quantitative drug levels to confirm exposure, the clinical course of both patients is consistent with the known 
effects of bupropion in overdose. In addition, in the first case, the effect of escitalopram may have also accounted for the observed clinical deterioration.

Conclusion: In addition to supportive care, early initiation of ECMO before cardiovascular collapse may be a consideration in severe overdoses involving bupropion.

\section{Serotonin Syndrome Resulting from Bupropion Overdose Treated with Cyproheptadine}

Waleed Alsukaiti ${ }^{1,2}$, Brian Murray ${ }^{1,2}$, Joseph Carpenter ${ }^{1,2}$, Camille Dunkley $^{1,2}$, Ziad Kazzi $^{1}$

${ }^{1}$ Emory University School of Medicine, Atlanta, GA, USA. ${ }^{2}$ Georgia Poison Center, Atlanta, GA, USA

Background: Bupropion increases the release of norepinephrine in the locus coeruleus and dorsal raphe which leads to an increase in the frequency with which serotonergic neurons fire. Overdoses from bupropion can cause a sympathomimetic toxidrome and seizures. Serotonin syndrome resulting from single agent bupropion overdose is controversial, and previously there has been only one reported case.

Case: A 14-year-old male with a history of depression ingested an unknown amount of bupropion. He presented in status epilepticus with vitals HR 86-178 bpm, BP 84-164/45-111 mmHg, RR 31/min, and T $37.7^{\circ} \mathrm{C}$. He received lorazepam with cessation of seizures, was intubated, and started on midazolam and fentanyl $(50 \mu \mathrm{g} / \mathrm{h})$. Fentanyl was replaced after $12 \mathrm{~h}$ with dexmedetomidine. On examination, he had inducible bilateral ankle clonus (5-6 beats) and was able to follow commands. Serotonin syndrome was suspected; therefore, he received two doses of cyproheptadine $4 \mathrm{mg}$. Within $2 \mathrm{~h}$ of the second dose, his clonus resolved. Serum concentrations of bupropion and hydroxybupropion were $15 \mathrm{ng} /$ $\mathrm{mL}$ and $960 \mathrm{ng} / \mathrm{mL}$, respectively.

Discussion: One case report and a recent ToxIC registry abstract have implicated bupropion as a cause of serotonin syndrome, with fentanyl as a possible contributing agent. Our patient developed autonomic instability, altered mental status, agitation, inducible clonus, and had temporal symptom resolution after receiving cyproheptadine, a serotonin antagonist, and a treatment not reported in prior studies. These findings provide further support for a serotonergic role of bupropion.

Conclusion: This case adds to the current literature on the role bupropion combined with fentanyl plays in developing serotonin syndrome. Further investigation is warranted to characterize the factors involved in the occurrence of serotonin syndrome in patients with bupropion overdose.

026. Does Co-Ingestion with Benzodiazepines Reduce the Rate of Seizures in Overdose? A Review of the Toxicology Investigators Consortium Database

Tony Rianprakaisang ${ }^{1,2}$, Colin Prather $^{1}$, Adam Blumenberg ${ }^{1,2}$, Lauren Murphy $^{1,2}$, Adrienne Hughes ${ }^{1,2}$, Robert Hendrickson ${ }^{1,2}$

${ }^{1}$ Oregon Health and Science University, Portland, OR. ${ }^{2}$ Oregon Poison Center, Portland, OR

Background: While benzodiazepines are first line treatments for seizures and serotonin toxicity, they are also common co-ingestants in overdose. Objective: Determine if co-ingestion of benzodiazepines decreases the rate of seizures or serotonin toxicity in common epileptogenic and serotonergic overdoses.

Methods: We queried the ToxIC database for ingestions of TCA's, bupropion, citalopram, and venlafaxine. Cases were separated according to presence or absence of benzodiazepine co-ingestion, and clinical features were compared.

Results: In bupropion overdose, co-ingestion of benzodiazepines was associated with lower rates of seizures (OR 0.19, CI 0.07-0.47) and tachycardia (OR 0.33 , CI $0.14-0.77$ ), but higher rates of CNS (OR 3.6, CI 2.16-6.11) and respiratory depression (OR 3.4, CI 1.68-6.87). In citalopram overdose, co-ingestion of benzodiazepines was associated with lower rates of seizures (OR 0.32 , CI $0.15-0.68$ ), serotonin toxicity (OR 0.40, CI 0.21-0.77), tachycardia (OR 0.26, CI 0.11-0.60), and hyperreflexia/myoclonus (OR 0.30 , CI $0.16-0.57$ ), but higher rates of CNS depression (OR 3.06, CI 2.06-4.54). In venlafaxine overdose, coingestion of benzodiazepines was associated with lower rates of seizures (OR 0.18, CI 0.04-0.76) and tachycardia (OR 0.14, CI 0.04-0.46), but higher rates of CNS depression (OR 2.66, CI 1.65-4.30). In TCA overdose, co-ingestion of benzodiazepines was only associated with lower rate of tachycardia (OR 0.28 , CI $0.12-0.65$ ) and agitation (OR $0.31, \mathrm{CI}$ 0.15-0.65), but not increased rates of CNS depression.

Discussion: This study demonstrates co-ingestion of benzodiazepines may decrease rates of seizures in several common epileptogenic overdoses. However, in most overdoses, benzodiazepine co-ingestion also increased rates of CNS depression. Additionally, concomitant benzodiazepine ingestion in citalopram overdose seems to have no effect on serotonin toxicity.

Conclusion: Benzodiazepine co-ingestion may offer some protection against seizures in select overdoses but may increase rates of CNS depression.

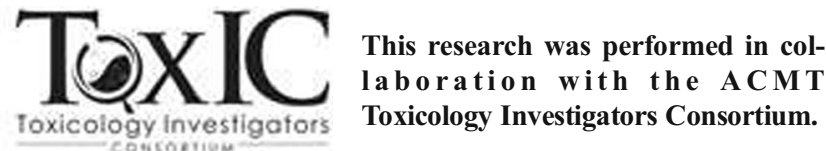

027. The Increasing Rate of Intentional Diphenhydramine Exposures in the USA-Another Emerging Public Health Concern

Antonia Nemanich ${ }^{1}$, Amber Sabbatini $^{1}$, Erica Liebelt ${ }^{2}$

${ }^{1}$ University of Washington, Seattle, WA, USA. ${ }^{2}$ Washington Poison Center, Seattle, WA, USA

Background: Suicide, medication overdose, and abuse/misuse of prescription medications and illicit substances have been increasing over the past 10 years. Diphenhydramine (DPH) is an overthe-counter medication that is easily obtainable, perhaps increasing its potential for misuse.

Objective: To describe and compare the characteristics and trends of intentional exposures to DPH among individuals 10 years of age and older reported to US poison control centers from 2005 to 2016.

Methods: National Poison Data System data from 2005 through 2016 were retrospectively analyzed to examine intentional DPH medication exposures. Intentional exposures included suicide, misuse, and abuse.

Results: From 2005 through 2016, there were 157,236 total intentional DPH exposures reported. Females accounted for more exposures, $63.7 \%$. From 2005 to 2016, intentional exposures increased from 33.7 per 100,000 to 61.1 per 100,000 ( $81 \%$ relative change). Increasing trends were most marked in the 10-14-year-old (yo) and $>65$ yo age ranges, which showed a $273 \%$ and $364 \%$ increase, respectively. The increase in the 10-14 age range was predominantly due to suicide attempts $(143 \%$ increase), while the increase in the $>65$ age range was predominantly due to misuse/abuse ( $275 \%$ increase). Single substance DPH vs. polysubstance exposures including DPH did not show any substantial trend changes. The percent of DPH exposures classified as moderate or severe has increased over the 2005-2016 period by $55.3 \%$ and $61.2 \%$ respectively, at the expense of minor exposures, which have decreased by $16.2 \%$. Major adverse clinical effects have increased $29.1 \%$ across all age groups. There were 741 reported deaths between 2005 and 2016. Deaths increased by $123.1 \%$ across all age groups.

Conclusions: Intentional exposures to DPH among all individuals $\geq$ 10 yo is an increasing problem in the USA. Of particular concern is the increasing trend in suicidal intent with DPH in individuals aged 10-14, and the increasing misuse/abuse in individuals $>65$. 
028. Shall We Try Angiotensin II (Too)? Treatment of Refractory Shock Due to Co-ingestion of Amlodipine and Benazepril: a Case Report

Gloria Gutierrez ${ }^{1,2}$, Christopher Dayton ${ }^{2}$, Lucas Smedley ${ }^{1,2}$, Hairitha Saikumar $^{2}$, Christopher Everett ${ }^{2}$, Abraham Rodriguez ${ }^{2}$, Rebecca Attridge ${ }^{2,3}$ ${ }^{1}$ University Health System, San Antonio, Texas, USA. ${ }^{2}$ University of Texas Health San Antonio, San Antonio, Texas, USA. ${ }^{3}$ University of the Incarnate Word Feik School of Pharmacy, San Antonio, Texas, USA

Background: Co-ingestion overdoses of antihypertensive medications cause profound circulatory shock that can be refractory despite multiple interventions.

Hypothesis: Angiotensin II causes rapid reversal of refractory shock due to co-ingestion of a calcium channel blocker and an angiotensinconverting enzyme inhibitor (ACE-I).

Methods: This is a single patient chart review. A 57-year-old male with a history of hypertension, depression, and alcohol abuse presented to the emergency department after ingesting amlodipine and benazepril in a suicide attempt. The patient's early course was complicated with acute renal insufficiency, a brief cardiac arrest, and persistent severe shock. He received $4 \mathrm{~g}$ of calcium gluconate and $10 \mathrm{mg}$ of glucagon without response. He was initiated on high-dose euglycemia therapy with insulin at 3.5 units $/ \mathrm{kg} / \mathrm{h}$. A $1.5 \mathrm{~mL} / \mathrm{kg}$ bolus of $20 \%$ lipid emulsion was administered twice with minimal improvement in mean arterial pressure (MAP). To maintain acceptable MAP, the patient required escalating doses of norepinephrine, epinephrine, and vasopressin. At this point, treatment was shifted to target the benazepril overdose and angiotensin II was started at $20 \mathrm{ng} / \mathrm{kg} / \mathrm{min}$ and titrated up to $80 \mathrm{ng} / \mathrm{kg} / \mathrm{min}$ over $2 \mathrm{~h}$.

Results: Within $2 \mathrm{~h}$ of initiating angiotensin II, epinephrine was weaned off and norepinephrine decreased by $50 \%$. His serum creatinine peaked at $2.70 \mathrm{mg} / \mathrm{dL}$ at angiotensin II initiation and returned to baseline within $12 \mathrm{~h}$. By day 4 of hospitalization, angiotensin II was discontinued, followed shortly by the other vasopressors. He was extubated on day 8 and demonstrated no persistent end-organ failure.

Discussion: This case demonstrates rapid reversal of shock and renal dysfunction with clear temporal relation to initiation of angiotensin II. Angiotensin II should be considered a therapeutic option for patients with severe shock in co-ingestions that include an ACE-I.

Conclusion: This case report illustrates the successful utilization of angiotensin II for refractory shock after co-ingestion of amlodipine and benazepril.

029. Acute Fondaparinux (Arixtra) Intentional Overdose via Selfinjection: a Case Report

Andrew Koons, Kenneth Katz, Matthew Cook, Robert Cannon, Gillian Beauchamp, Ryan Surmaitis

Lehigh Valley Health Network/University of South Florida Morsani College of Medicine, Allentown, PA, USA

Background: Factor Xa inhibitors - antithrombin III inhibitor anticoagulants indicated for venous thrombosis and pulmonary embolism - are widely prescribed, and adverse hematologic effects have been described in this setting. However, there are no current reports of intentional fondaparinux poisoning by self-injection.

Methods: A novel case of fondaparinux overdose by self-injection with serum concentration is reported. A 49-year-old woman with a history of stiff person syndrome, recurrent pulmonary embolism, and depression prescribed fondaparinux presented to ED after intentionally injecting herself with 13 syringes of her fondaparinux. She reported injecting them subcutaneously in her abdomen over the course of several minutes about $18 \mathrm{~h}$ before coming to ED. Each syringe contained $7.5 \mathrm{mg}$ fondaparinux (total $97.5 \mathrm{mg}$ ). Her vital signs and laboratory testing were unremarkable (including routine toxicologic, renal, and coagulation studies).

Results: The patient was admitted given no current recommendations regarding this poisoning and the potential for depot effect of large amounts of fondaparinux which could be absorbed. A serum anti-Xa fondaparinux concentration the day after admission measured $>4.0 \mathrm{mg} /$ $\mathrm{L}$ (therapeutic $0.50 \mathrm{mg} / \mathrm{L}$ ). Physical exam showed multiple abdominal injection sites without excessive bleeding. She demonstrated no signs of bleeding in the hospital and was discharged to an inpatient psychiatric unit without sequelae on hospital day three.

Discussion: There are currently no reported cases of intentional fondaparinux poisoning, and this patient had an uneventful course. Pharmacokinetics of the drug indicates rapid absorption after subcutaneous injection with peak concentrations within two hours. It is not metabolized and undergoes renal elimination unchanged. Large doses may have a depot effect with unknown toxicokinetics and potential for delayed complications.

Conclusion: Given the absence of consensus recommendations at this point, the authors recommend prolonged observation with intentional poisoning given the difficulty of hematologic monitoring, especially in those patients with abnormal renal function or evidence of bleeding.

\section{Topiramate Toxicity with Delayed Tonic-Clonic Seizure in the} Absence of Non-Gap Metabolic Acidosis

Alexander Sidlak ${ }^{1}$, Ryan Marino ${ }^{2}$, Joseph Yanta ${ }^{2}$

${ }^{1}$ University of Pittsburgh School of Medicine, Pittsburgh, PA, USA. ${ }^{2}$ University of Pittsburgh Medical Center, Pittsburgh, PA, USA

Background: Topiramate, a second-generation antiepileptic, is increasingly used for indications apart from epilepsy. Its mechanism of action is not entirely clear, but it is known to enhance gamma-aminobutyric acid (GABA) conduction, and inhibit glutamate conduction and carbonic anhydrase activity. This latter effect produces a non-gap metabolic acidosis in up to $48 \%$ of patients at therapeutic dosage. In overdose, some case series show that a non-gap acidosis is dose-related and uniformly present if severe symptoms occur. Hypothesis: Severe topiramate toxicity can occur without a non-gap acidosis.

Methods: This is a single patient case report. A 14-year-old girl presented to the emergency department after overdosing on $18 \mathrm{~g}(>400 \mathrm{mg} / \mathrm{kg})$ of topiramate. She was taking this for migraines and had no history of seizures. On arrival, she was somnolent, nonverbal, and without focal neurologic deficit or vital sign abnormality. She had no anion gap and a normal bicarbonate. Two hours into her stay, she had a generalized tonicclonic seizure.

Results: Her topiramate level was $105.7 \mu \mathrm{g} / \mathrm{mL}$ initially and $>160 \mu \mathrm{g} /$ $\mathrm{mL} 12 \mathrm{~h}$ later. She was admitted, and her mental status slowly normalized over two days. At no point during her stay did she develop a hyperchloremic metabolic acidosis.

Discussion: This patient had marked elevation in her topiramate level and seized, yet her metabolic profile remained normal. A characteristic non-anion gap metabolic acidosis did not develop. In prior case reports, a non-gap acidosis has been present in every case of coma or seizure and it persists after the resolution of toxic encephalopathy.

Conclusion: Significant topiramate toxicity with seizures can occur without the development of a hyperchloremic metabolic acidosis. Given the risk of seizure, continued observation based on initial toxic encephalopathy even in the absence of suggestive laboratory findings is recommended when levels are not readily available.

\section{Acute Sulodexide Overdose Without Bleeding Complication: a Case Report}

Aynur Sahin ${ }^{1,2}$, Shaikhah Alotiabi ${ }^{1,3},{\text { Sandra } \text { Mrad }^{4}, \text { Zeina Mneimneh }}^{4}$, Tharwat El Zahran ${ }^{4}$, Ziad Kazzi ${ }^{1}$

${ }^{1}$ Emory University School of Medicine, Atlanta, Georgia, USA.

${ }^{2}$ Karadeniz Technical University School of Medicine, Trabzon, Turkey.

${ }^{3}$ King Fahd University Hospital, Alkhobar, Saudi Arabia. ${ }^{4}$ American University of Beirut Medical Center, Beirut, Lebanon 
Background: Sulodexide is a heparinoid substance consisting of a mixture of low-molecular-weight heparin and dermatan sulfate which is used as a hypolipidemic and antithrombotic for peripheral vascular and cerebrovascular diseases. There is a lack of information about its toxicity in acute overdose.

Case report: A 92-year-old male with a history of hypertension, diabetes and myocardial infarction intentionally ingested 24 tablets of a $500 \mathrm{mg}$ acetaminophen, 35 tablets of a 250 lipoprotein lipase releasing units (LSU) sulodexide $1 \mathrm{~h}$ before arrival to the emergency department. He admitted to the ingestion, and his family brought the empty boxes of his medication. His physical examination was unremarkable. All laboratory values were within normal limits, and his 4-h APAP level was $49.2 \mathrm{mg} / \mathrm{L}$. ECG showed a sinus rhythm with first degree AV block. He was admitted to ICU to observe any bleeding complication due to acute sulodexide overdose. His hemogram test and anticoagulation parameters (PT, PTT, INR) were normal during his observation. No bleeding complications were observed; however, mild hyponatremia (Na: $124 \mathrm{mmol} / \mathrm{L}$ ) developed, and he was discharged on the third day of his admission in normal condition.

Case discussion: The pharmacological effects of sulodexide differ from other anticoagulants and are mainly characterized by a good oral absorption, prolonged half-life $(25.8 \pm 1.9 \mathrm{~h})$ and reduced impact on global coagulation and bleeding parameters (PT, PTT). It is reported to have minimal systemic side effects. However, there are no reported cases of its acute overdose.

Conclusion: This is the first case report of acute sulodexide overdose, and according to the findings, supratherapeutic doses of sulodexide are well tolerated.

\section{Acute Lethal Fluvoxamine Overdose with Postmortem Levels}

Jenna Otter $^{1}$, Iain McIntyre ${ }^{2}$, Jacquelyn Morhaime ${ }^{3}$, F Lee Cantrell $^{1}$

${ }^{1}$ UCSD, San Diego, CA, USA. ${ }^{2}$ Department of Medical Examiner, San Diego, CA, USA. ${ }^{3}$ County of San Diego Medical Examiner's Office, San Diego, CA, USA

Background: Fluvoxamine is a selective serotonin reuptake inhibitor that has been considered relatively safe in overdose. Most cases of mortality from fluvoxamine involve multiple substances. At therapeutic and supratherapeutic concentrations, fluvoxamine itself affects cardiac conduction, prolongs QTc interval, causes hypotension and obtundation, and can increase propensity for seizures. Isolated overdoses of fluvoxamine resulting in death are exceedingly rare.

Research Question: Can fluvoxamine, a medication with high safety profile, be a cause of death in isolated overdose?

Methods: This is a case report of a 62-year-old man with a history of depression who was found dead at his home from presumed suicide. His only medication prescribed was fluvoxamine. The subject underwent autopsy shortly after death, in which postmortem blood from the common iliac veins and liver tissue was analyzed using gas chromatography-flame ionization detection, enzyme linked immunosorbent assay for a panel of 13 drugs of abuse, and alkaline drug screen by gas chromatography-mass-spectrometry. Fluvoxamine detection was confirmed and quantified. At autopsy, he had no other major injuries, and other findings included arteriolonephrosclerosis and mild cardiac hypertrophy.

Results: Peripheral blood and liver analysis revealed fluvoxamine concentrations of $4.9 \mathrm{mg} / \mathrm{L}$ and $440 \mathrm{mg} / \mathrm{kg}$ respectively. There was no ethanol (or simple volatiles), illicit, or other drugs identified. His cause of death was determined to be acute fluvoxamine toxicity.

Conclusion: We report a case of isolated fluvoxamine overdose resulting in death. Compared to levels reported in the literature, the subject had exceedingly elevated postmortem fluvoxamine concentrations. Mechanism of death is conjectured to be related to the cardiac effects of fluvoxamine in overdose either alone or in combination with underlying medical causes.
033. Hemodialysis: the Final Frontier for Acute Lacosamide Toxicity?

Emily Kiernan ${ }^{1}$, Sukhshant K Atti ${ }^{1}$, Elizabeth Silver $^{2}$, Mark R Layer ${ }^{1}$, Melissa Gittinger ${ }^{1,2}$

${ }^{1}$ Emory University School of Medicine, Atlanta , GA, USA. ${ }^{2}$ Georgia Poison Center, Atlanta, GA, USA

Background: Lacosamide is an antiepileptic medication that acts to enhance slow inactivation of voltage-gated sodium channels and stabilize neuronal transmembrane electrical potential. Although initially marketed as having minimal toxicity, there is mounting evidence in the literature documenting cardiac and neurologic adverse effects in overdose. However, pharmacokinetic data suggest that lacosamide is amenable to hemodialysis due to its low volume of distribution, low molecular weight, and minimal protein binding.

Hypothesis: Patients that present to the ED with cardiac toxicity and neurotoxicity from acute lacosamide ingestion can be treated with hemodialysis for clinical improvement.

Methods: This is a case report of a 61-year-old female with a history of seizure disorder and chronic kidney disease on intermittent hemodialysis, who intentionally ingested a handful of lacosamide $200 \mathrm{mg}$ tablets. She arrived at the ED about $1 \mathrm{~h}$ post-ingestion and was obtunded.

Results: The patient's initial labs showed a metabolic acidosis, renal failure, and undetectable ethanol, phenytoin, and valproic acid levels. Initial ECG showed normal sinus rhythm, rate 78, PR $211 \mathrm{~ms}$, QRS $143 \mathrm{~ms}$, QTc $488 \mathrm{~ms}$. She received two bicarbonate $50 \mathrm{mEq}$ IV boluses over $2 \mathrm{~h}$ and then a bicarbonate continuous infusion was started, which did not significantly affect her ECG or mental status. Approximately $10 \mathrm{~h}$ after arrival, the patient was started on hemodialysis. A repeat ECG after $3 \mathrm{~h}$ of hemodialysis demonstrated a normal sinus rhythm, QRS $108 \mathrm{~ms}$, QTc $458 \mathrm{~ms}$, PR $160 \mathrm{~ms}$, and her neurologic exam returned to baseline. A lacosamide level from presentation resulted at $50.3 \mu \mathrm{g} / \mathrm{mL}$ (therapeutic $6.6-18.3 \mu \mathrm{g} / \mathrm{mL}$ ). The patient was discharged on hospital day 2 at her baseline clinical status.

Conclusion: Patients who demonstrate cardiac or neurologic dysfunction should be considered for hemodialysis as this may improve their clinical status and potentially decrease hospital length of stay and hospitalacquired complications.

\section{Racial and Ethnic Patterns of Intentional Overdose}

Alexa Camarena-Michel ${ }^{1,2}$, Benjamin Hatten ${ }^{1,2}$

${ }^{1}$ Denver Health, Denver, CO, USA. ${ }^{2}$ University of Colorado, Denver, CO, USA

Background: Scant information exists evaluating patterns of pharmacologic overdose across racial and ethnic groups. Our research group recently performed a preliminary analysis on a smaller sample size in an attempt to answer the following: Are certain populations at greater risk of intentional overdose, self-harm or misuse? By expanding the sample size and analysis, we will identify populations that may be more vulnerable to intentional overdose.

Hypothesis or Research Question: Are there identifiable patterns of overdose across ethnic and racial groups?

Methods: This was a case-control study using the Toxicology Investigators Consortium (ToxIC) Registry. In this analysis, intentional overdoses from 2017 to 2018 were evaluated including Hispanics vs. non-Hispanics as well as six racial groups: American Indian/Alaska Native, Asian, Black, Caucasian, Pacific Islander, mixed/unknown. Additionally, intentional vs. unintentional overdoses and reasons for self-harm by pharmacologic agent were analyzed across various races and ethnicities. Specifically, five categories were considered: attempted self-harm, misuse, adverse effects, drug concealment, and unknown.

Results: Of the 7,577 cases evaluated, an association between racial group and intentional vs. unintentional overdose was identified (American Indian/Alaska Native $78 \%$ vs. $22 \%$, Asian $61 \%$ vs. $39 \%$, 
Black $76 \%$ vs. $24 \%$, Caucasian $79 \%$ vs. $21 \%$, Pacific Islander $100 \%$, unknown/mixed $74 \%$ vs. $26 \%, p<0.01)$. There was also significant correlation between race and motives of intentional self-harm $(p<0.01)$. Similar rates of death were observed when comparing Hispanics and non-Hispanics $(0.95 \%$ and $1.38 \%, p=0.19)$ and between the six racial categories (range $0-1.6 \%, p=0.83$ ).

Conclusion: A more robust analysis of data evaluating racial and ethnic characteristics of intentional overdoses reveals the need for ongoing investigation. With the inclusion of more cases, this analysis further elucidates the interplay between race, ethnicity and patterns of overdose. Ongoing analysis will help identify vulnerable patient populations and inform public health efforts.

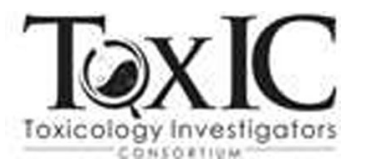

This research was performed in collaboration with the ACMT Toxicology Investigators Consortium.

\section{An Unusual Cause of Transient Blindness}

James Krueger, J Kowalski, Steven Walsh, Sarah Weiss Einstein Medical Center, Philadelphia, PA, USA

Background: Heroin is often adulterated in order to increase profit and potentiate effect. Co-ingestion of adulterants may have deleterious side effects and alter clinical presentation.

Case Report: A 32-year-old man presented to an emergency department with complaints of acute vision change. He denied past medical history, took no prescription or over-the-counter medications. He insufflated five bags of heroin $5 \mathrm{~h}$ prior to evaluation. He developed blurred vision, which worsened with time. Physical exam is unremarkable aside from minimally reactive pupils and reported blindness. CT head and MRI were nondiagnostic. Labs were obtained and normal. Ophthalmology and neurology were consulted which confirmed initial physical exam results. Ophthalmology noted normal maculae without signs of edema. Patient was admitted for further workup. Toxicology was consulted and ordered a quinine concentration, resulting at $0.13 \mathrm{mg} / \mathrm{L}(0-0.1 \mathrm{mg} / \mathrm{L})$.

Discussion: Historically, quinine was thought to have been added to heroin in New York City during the 1930-40s. Currently, the use of quinine as a bittering agent has obscured the quality of heroin to users. Supratherapeutic administration of ingested or intravenous quinine is usually associated with toxicity. The average reported serum concentration associated with transient blindness is $10 \mathrm{mg} / \mathrm{L}$ with $15 \mathrm{mg} / \mathrm{L}$ causing permanent blindness. Our patient's level was significantly lower than these concentrations, $0.13 \mathrm{mg} / \mathrm{L}$. His quinine level was assessed $14 \mathrm{~h}$ after reported insufflation, which may account for a lower than expected level. The proposed mechanism of quinine retinal toxicity includes an indirect mechanism of ischemic retinopathy via central retinal artery spasm versus a direct neuro-retina, ganglion cell layer toxin.

Conclusion: After literature review, this may represent the first case of insufflated quinine-tainted opioids associated with vision loss. The direct passage along the cribriform plate may allow for lower concentrations to cause blindness not seen in oral or IV toxicity.

036. Management of Patients with Acute Drug Overdose in an Emergency Department Observation Unit

Siri Shastry ${ }^{1}$, Lynne Richardson ${ }^{1}$, Jonathan Yeo $^{1}$, Alex Manini ${ }^{2}$ ${ }^{1}$ Department of Emergency Medicine, Icahn School of Medicine at Mount Sinai, Mount Sinai Hospital, New York, NY. ${ }^{2}$ Division of Medical Toxicology, Department of Emergency Medicine, Icahn School of Medicine, Elmhurst Hospital Center, New York, NY

Background: Emergency Department (ED) observation units (OU) are being increasingly used to optimize care and have been shown to decrease costs. Evidence to support the use of OUs in the management of drug overdose is limited.

Hypothesis: We hypothesized that OU management of low-risk patients with drug overdose would result in acceptably low rates of adverse events and 30-day bouncebacks.

Methods: This was a secondary data analysis of a prospective consecutive cohort of ED patients presenting to a single, academic, tertiary care center from March 2015 to present. Patients with acute drug overdose were screened and included if deemed low-risk for management in the $\mathrm{OU}$ as per the primary team in accordance with an established OU overdose protocol. Exclusion criteria for the cohort included age $<18$, nondrug exposure, alternative diagnosis, chronic toxicity, or lacking data. The OU protocol further excluded the following: hemodynamic instability, ECG abnormality, electrolyte imbalance, altered mental status, recurrent seizures, persistent toxic drug level, and high psychiatric risk. The primary study composite outcome was occurrence of advanced airway intervention, adverse cardiovascular events or in-hospital mortality. Secondary outcomes were disposition and return visits.

Results: Of 947 ED overdose patients screened, 641 were included in the cohort, and of these, 25 (3.9\%) were managed in the OU. Out of 25 OU patients, 23 patients $(92 \%)$ were discharged home and did not require admission. No patients required airway management and only two patients $(8 \%)$ experienced an adverse cardiovascular event (myocardial injury) with no deaths. Three patients $(12 \%)$ returned to the hospital within 30 days.

Conclusion: While only a small proportion of ED overdose patients were eligible for OU management, our data support the safety of an OU protocol for select patients. Further studies are warranted to improve OU management of low risk ED patients with acute drug overdose.

\section{Acute Stroke After Ethylene Glycol Ingestion}

Ashley Carter Powell ${ }^{1,2}$, Laura Tortora ${ }^{1,2}$, Joshua Canning ${ }^{1,2}$

${ }^{1}$ Department of Medical Toxicology, Banner-University Medical Center Phoenix, Phoenix, Arizona, USA. ${ }^{2}$ Division of Medical Toxicology and Precision Medicine, University of Arizona College of Medicine Phoenix, Phoenix, Arizona, USA

Background: Ethylene glycol (EG) toxicity is a well-known cause of renal failure. While several neurological manifestations have been reported, acute stroke is a very rare finding.

Methods: This is a case report. A 55-year-old male presented to an emergency department (ED) via EMS with altered mental status. He reported ethanol and fentanyl abuse but was unable to provide additional history. He required emergent intubation for worsening CNS depression. Laboratory studies revealed: $\mathrm{CO}_{2} 3 \mathrm{mmol} / \mathrm{L}$, anion gap $26 \mathrm{mmol} / \mathrm{L}$, creatinine $1.7 \mathrm{mg} / \mathrm{dL}$, osmolal gap $53 \mathrm{mOsm} / \mathrm{kg}$, and undetectable ethanol level. He received fomepizole for suspected toxic alcohol ingestion and was transferred for ICU admission. CT head without contrast was obtained prior to transfer and showed no acute abnormalities. EG level obtained on admission was $179 \mathrm{mg} / \mathrm{dL}$. He was started on emergent hemodialysis, which was continued until EG level was undetectable.

Results: Extubation attempt was unsuccessful due to persistently poor mental status and concern for aspiration. After reintubation, CT head was repeated and showed acute right-sided cortical infarcts. MR brain was consistent with an embolic pattern of ischemia in the right parietal, occipital, and temporal lobes with small affected areas scattered bilaterally. Stroke Neurology was consulted and attributed the unusual location of the infarcts to sequelae from the EG ingestion as this has been reported, rarely, in the literature. He has remained dialysis- and ventilatordependent.

Discussion: Neurological manifestations of EG usually include CNS depression, cerebral edema, and cranial nerve dysfunction. Midbrain and brainstem abnormalities on CT have also been reported. Acute stroke is a rare manifestation that is hypothesized to occur due to precipitation of calcium oxalate crystals in cerebral blood vessels. 
Conclusion: Acute stroke is a rare sequela of EG ingestion and should be considered in patients with persistently altered mentation in the setting of toxicity.

038. A Mother and Child Confusion Is Only a Motion Away: Accidental Promethazine Overdose in a Newborn

Michael Veltre, Cynthia Santos, Bruce Ruck, Lewis Nelson, Diane Calello

Rutgers New Jersey Medical School, Newark, NJ, USA

Background: Promethazine is a competitive histamine- 1 antagonist that is used for its sedative, antiemetic, and anticholinergic effects, and is contraindicated in patients under the age of two due to potential respiratory depression. There is limited evidence regarding promethazine overdose in pediatric patients, especially in neonates.

Methods: This is a single chart review. A 1-h-old $2.81 \mathrm{~kg}$ girl was unintentionally administered a $25 \mathrm{mg}$ injection IM $(8.9 \mathrm{mg} / \mathrm{kg}$ dose $)$ of promethazine that was intended for the mother. The regional poison center recommended to monitor for respiratory depression, anticholinergic effects, seizure activity, and ECG abnormalities.

Results: Initial vital signs were HR 147, BP 61/42, RR 36, $\mathrm{SpO}_{2} 98 \%$ (RA), temp $35.5^{\circ} \mathrm{C}$. ECG: normal sinus rhythm, rate 147 , QRS $50 \mathrm{~ms}$, and QTc $444 \mathrm{~ms}$. In the NICU, she developed somnolence and shallow respirations, and her ECG demonstrated some ectopic beats, but these effects resolved overnight. She had poor feeding and received 3 days of nasogastric tube feeds, but was advanced to full PO feeding without sequelae. She did not develop other effects including apnea, seizures, tachycardia, hypotension, or dysrhythmias.

Discussion: Sedation, hallucinations, and tachycardia following parenteral promethazine have been reported at $1.1 \mathrm{mg} / \mathrm{kg}$ in pediatric patients. There is one reported death in a 2-year-old child who ingested 200$300 \mathrm{mg}$ of promethazine tablets (approx. $17-25 \mathrm{mg} / \mathrm{kg}$ ) [Dominikovich, 1962]. The paucity of evidence regarding infant exposures to promethazine makes management and risk assessment difficult. In this case, a high dose was generally well tolerated, although supportive care and observation were required.

Conclusion: Neonates with promethazine overdose, most likely from a delivery room error, should be monitored for respiratory depression, ECG abnormalities, poor feeding, and other potential adverse effects. In this case, $8.9 \mathrm{mg} / \mathrm{kg}$ produced some central nervous system and respiratory effects but resolved favorably with supportive care.

\section{The "Not So" Perilous Unintentional Semaglutide Overdose}

Camille Dunkley $^{1,2}$, Brian Murray ${ }^{1,2}$, Waleed Alsukaiti ${ }^{1,2}$, Melissa Gittinger $^{1,2}$

${ }^{1}$ Emory University School of Medicine, Atlanta, Georgia, USA. ${ }^{2}$ Georgia Poison Center, Atlanta, Georgia, USA

Background: Catastrophic clinical effects can occur after unintentional supratherapeutic dosage errors; however, some effects are minor. Documentation of all errors is important as they assist in establishing triage safety limits, which is important for poison centers. Antidiabetic medications are worrisome due to the persistent hypoglycemia that occurs after exposure to certain agents. As new classes are constantly emerging, there are limited reported data of clinical effects after supratherapeutic dosing.

Methods: This is a single-patient case report. A 69-year-old female with a history of diabetes presented to the ED complaining of nausea and chest pain for one day. She reported accidentally injecting a 2-month supply $(8 \times$ recommended dose $)$ of her medication, semaglutide. She denied coingestions. Serum serial glucose measurements were $100 \mathrm{mg} / \mathrm{dL}, 97 \mathrm{mg} /$ $\mathrm{dL}, 122 \mathrm{mg} / \mathrm{dL}$, respectively. Creatinine was elevated $1.5 \mathrm{mg} / \mathrm{dL}$, and lipase was within normal limits. The patient was subsequently admitted for observation.
Results: Overnight, she experienced continued nausea but tolerated oral intake. Blood glucose remained above $90 \mathrm{mg} / \mathrm{dL}$ throughout the admission. She was discharged on hospital day 2 .

Discussion: Semaglutide is a GLP-1 receptor agonist approved for treatment of type 2 diabetes. It works by stimulating insulin release from pancreatic beta cells, decreasing gastric emptying, and increasing satiety. Adverse effects described with therapeutic doses include nausea, vomiting, diarrhea, abdominal pain, pancreatitis, and worsening renal insufficiency. To our knowledge, there are no reported data after overdoses. The risk of hypoglycemia after semaglutide overdose appears to be low based on information from other GLP-1 receptor agonist (with differing pharmacokinetics) compared to other classes of antidiabetic medications. Management should be supportive and directed towards relieving symptoms.

Conclusion: It is vital for healthcare professionals to be diligent with reporting all dosage errors, especially ones that are exceedingly above the recommended dose, regardless of the clinical effects, to strengthen the current literature and establish safety limits

040. Toxic Encephalopathy, Anion Gap Metabolic Acidosis, and Seizure Secondary to Naproxen Poisoning: a Case Report

John Jacobs, Jr. ${ }^{1}$, Robert Avera ${ }^{1,2}$, Mary Wermuth ${ }^{1,2}$

${ }^{1}$ Indiana University School of Medicine, Indianapolis, IN, USA. ${ }^{2}$ Indiana Poison Control Center, Indianapolis, IN, USA

Background: CNS toxicity has been reported in NSAID overdose; however, seizure has been only infrequently reported following naproxen sodium ingestion. Hypothesis: In individuals with an excessive serum concentration of naproxen, CNS toxicity can result in seizure.

Methods: This is a single patient chart review. A 35-year-old female with past medical history significant for depression and prior suicide attempt with ingestion presented after ingestion of $30 \mathrm{~g}$ naproxen. Approximately $7.5 \mathrm{~h}$ after reported ingestion, patient developed progressively altered mental status and anion gap metabolic acidosis. Patient was witnessed to have seizure-like activity that ceased after lorazepam administration. Supportive care with IV fluids and benzodiazepines was continued, and a serum naproxen level was obtained.

Results: One serum naproxen level was obtained on the day of presentation, approximately $13 \mathrm{~h}$ after ingestion. The level was $750 \mu \mathrm{g} / \mathrm{mL}$ (reporting limit$30 \mu \mathrm{g} / \mathrm{mL}$, analgesic range $30-90 \mu \mathrm{g} / \mathrm{mL}$ ). Urine drug screen was positive for cannabis. The patient's known pharmacies were contacted, and state-wide controlled prescription database was reviewed without identifying any possible co-ingestions. Initial $\mathrm{CO}_{2}$ and anion gap were 17 and $16 \mathrm{mmol} / \mathrm{L}$, respectively. Lactate peaked at $10.8 \mathrm{mmol} / \mathrm{L}$. EEG on days 1 and 2 were without epileptiform focus or triphasic waves. The patient remained intubated for two days after initial presentation and had agitated delirium after extubation. The patient returned to baseline on hospital day 4 and was discharged to home on day 6 . Conclusion: To our knowledge, seizure has only been infrequently reported following significant naproxen over-ingestion. This report of seizure after naproxen ingestion adds to the paucity of literature regarding this potential toxic effect. Additionally, this patient remained altered for 4 days before full return to baseline, exhibiting a prolonged encephalopathy not previously described. While this is a single case report, this case adds further credibility towards having an increased awareness of this possible adverse event following large naproxen ingestions.

\section{Tejocote Raíz: a Root for Weight-loss or Digoxin Doppelgänger?}

Katherine Palmer ${ }^{1}$, Jacob Lebin ${ }^{1}$, Rebekah Burns ${ }^{2}$, Suzan Mazor ${ }^{2,3}$

${ }^{1}$ University of Washington, Department of Emergency Medicine, Seattle, WA, USA. ${ }^{2}$ University of Washington, Department of Pediatrics, Seattle, WA, USA. ${ }^{3}$ Washington Poison Center, Seattle, WA, USA

Background: Tejocote root, from a species of hawthorn (Crataegus mexicana), has been recently marketed as a weight-loss supplement. Several hawthorn species have been traditionally used in the treatment 
of heart disease and may have positive inotropic effects. However, little is known about hawthorn, and tejocote in particular, when consumed in excess. We describe a case of tejocote exposure from a weight-loss supplement resulting in severe cardiotoxicity.

Hypothesis: Excessive tejocote root exposure may result in cardiotoxicity.

Methods: This is a single case report of a tejocote ingestion. A healthy 16-year-old girl presented to an emergency department after ingesting eight pieces of her mother's 'Alipotec Tejocote Raiz' weight-loss supplement. At arrival, she was sleepy, had active vomiting and diarrhea, and a heart rate in the 40's. Her initial blood chemistries were unremarkable except for an elevated digoxin assay of $0.7 \mathrm{ng} / \mathrm{mL}$. All other drug screens were negative. She denied taking digoxin, and no family member was prescribed this medication. She was transferred to our emergency department after multiple episodes of hypopnea and persistent bradycardia. Her ECG demonstrated a heart rate of 38 and Mobitz type 2 second degree heart block. She was given one vial of Digoxin Immune Fab given this evidence of cardiotoxicity. Unfortunately, no clinical response was observed. Approximately $29 \mathrm{~h}$ after ingestion, subsequent ECGs demonstrated return to normal sinus rhythm, and her symptoms resolved.

Discussion: To our knowledge, this is the first reported case of toxicity from tejocote root, which resulted in cardiotoxicity and respiratory depression. Similar to other hawthorn species, tejocote appears to cross react with some commercial digoxin assays, resulting in a falsely elevated digoxin level. Clinically, tejocote root does not appear to respond to Digoxin Immune Fab.

Conclusions: Tejocote root may cross react with some digoxin assays, and overdose may cause cardiac dysrhythmias and respiratory depression.

\section{Fatality from Potassium Gold Cyanide Overdose: a Case Report}

Elizabeth Harmon, Jacob Lebin, David Murphy, Bjorn Watsjold University of Washington, Department of Emergency Medicine, Seattle, WA, USA

Background: Potassium gold cyanide is a compound used in industrial electroplating and found as a by-product of gold mining. Ingestion of this substance is rarely reported in the literature, and previous reports are limited to occupational exposure. We describe a fatal case of intentional potassium cyanide ingestion, despite antidotal therapy.

Hypothesis: Potassium gold cyanide ingestion may result in severe toxicity and multi-organ failure.

Methods: Single-patient chart review.

Case: An 84-year-old man presented after ingesting 0.5-1 teaspoon of potassium gold cyanide, containing approximately $0.4-0.8 \mathrm{~g}$ cyanide and 1.5-3 g gold, in an intentional suicide attempt. Prehospital providers found the patient with severe vomiting and diarrhea and administered $5 \mathrm{~g}$ of hydroxocobalamin. Despite antidotal therapy, the patient rapidly developed severe lactic acidosis, multi-organ dysfunction, and respiratory failure. After admission, the patient received an additional $10 \mathrm{~g}$ of hydroxocobalamin and $12.5 \mathrm{~g}$ of sodium thiosulfate. A serum cyanide level after $10 \mathrm{~g}$ of hydroxocobalamin was $0.25 \mathrm{~g} / \mathrm{L}$. The patient eventually went into cardiovascular collapse and expired $12 \mathrm{~h}$ after initial ingestion. Discussion: While potassium cyanide ingestion has been well described, the toxicity of potassium gold cyanide is less understood. The patient's clinical findings were consistent with acute cyanide poisoning, but the cyanide level was below the toxic level, although obtained after antidotal therapy. Two previous case reports describing potassium gold cyanide ingestion similarly had low or undetectable serum cyanide levels, implicating gold as a possible cause of acute toxicity. In addition to treatment of cyanide toxicity, management of acute gold toxicity should also be considered in acute potassium gold cyanide ingestion.

Conclusion: Potassium gold cyanide is a rare exposure that can result in end organ failure and fatality. The toxic effects may result from both cyanide and gold toxicity.
043. Falsely Elevated Salicylate Level in the Setting of Hyperlipidemia

Shirley Shao, Ryan Marino, Anthony Scoccimarro, Alexander Sidlak, Michael Abesamis

University of Pittsburgh School of Medicine, Pittsburgh, PA, USA

Background: Hyperlipidemia interferes with spectrophotometric assays and has been reported to cause falsely elevated salicylate levels. Hypothesis: Hyperlipidemia can falsely elevate salicylate levels.

Methods: This is a single patient case report. A 58-year-old man was diagnosed with pancreatitis at an outside hospital. An elevated salicylate level prompted transfer to a tertiary care facility. Salicylate levels were measured by enzymatic (salicylate hydroxylase) assay without a lipid reduction technique, such as a lipid-clearing agent or ultracentrifugation. At the tertiary care facility, repeat salicylate levels were measured with an enzyme multiplied immunoassay technique (Syva EMIT assay with VivaE system) after ultracentrifugation. The patient's blood was described as grossly lipemic in both facilities.

Results: At the outside hospital, the patient had a lipase of $8950 \mathrm{mg} / \mathrm{dL}$ and salicylate level of $31.2 \mathrm{mg} / \mathrm{dL}$. Repeat blood work at the tertiary care facility showed an undetectable salicylate level. The next day, patient had triglycerides $5099 \mathrm{mg} / \mathrm{dL}$, cholesterol $1055 \mathrm{mg} / \mathrm{dL}$, HDL $9 \mathrm{mg} / \mathrm{dL}$. LDL and VLDL could not be calculated. The patient denied taking salicylate containing products, such as bismuth salicylate, topical analgesics, or combination over-the-counter analgesics.

Discussion: Two prior cases describe falsely elevated salicylate levels in the setting of hyperlipidemia. Both cases initially used the enzymatic method for salicylate quantification without lipid reduction. In both cases, repeat salicylate levels were undetectable after lipid reduction with a lipid clearing agent (LipoClear). In this patient, ultracentrifugation of the sample removed the interference caused by lipids in the serum. No significant interference has been found with the Syva EMIT assay with triglycerides of up to $750 \mathrm{mg} / \mathrm{dL}$ based on manufacturer testing; however, it is unclear how triglycerides of $5099 \mathrm{mg} / \mathrm{dL}$ would affect the assay.

Conclusion: Hyperlipidemia causes spuriously elevated salicylate levels without lipid reduction or ultracentrifugation.

\section{Evidence of Compartment Syndrome in a Patient with Salicylate Toxicity}

Kim Kwai, Kelly Owen, Jonathan Ford

University of California, Davis, Sacramento, CA, USA

Background: Compartment syndrome is an uncommon complication of rhabdomyolysis. We describe a patient with salicylate toxicity who we believe developed compartment syndrome after an intentional overdose. Methods: The patient was a 13-year-old female, found unresponsive on the floor with a half-empty 500 tablet aspirin bottle. She presented to an outside ED, hyperthermic $40.2^{\circ} \mathrm{C}$, tachycardic 135 bpm, hypotensive 89/ 49 , tachypneic $30, \mathrm{spO}_{2} 100 \%$ on RA. Her physical exam was documented as unremarkable. She was then intubated and transferred to our hospital. Upon arrival $3.5 \mathrm{~h}$ later, she was hypotensive (MAP 62), tachycardic (160s), and hyperthermic $\left(40.4{ }^{\circ} \mathrm{C}\right)$. She was on sodium bicarbonate and vasopressor infusions. Pertinent labs: $\mathrm{ABG} \mathrm{pH} 7.15, \mathrm{pCO}_{2} 72, \mathrm{pO}_{2} 19$, with $\mathrm{CO}_{2} 13 \mathrm{mmol} / \mathrm{L}$, anion gap 26, lactic acid $2.4 \mathrm{mg} / \mathrm{dL}$. Salicylate $113 \mathrm{mg} / \mathrm{dL}(\sim 2$-h level) $\rightarrow 81.1 \mathrm{mg} / \mathrm{dL}$ ( $\sim$-h level). Acetaminophen, ethanol, and urine drug screen were undetectable/negative. Her repeat salicylate was $18.4 \mathrm{mg} / \mathrm{dL}$ after $6 \mathrm{~h}$ of hemodialysis, and she was extubated. The next day, she developed severe bilateral lower extremity pain and pitting edema. Her creatine kinase peaked at 144,117 U/L, AST $2898 \mathrm{IU} / \mathrm{L}$, ALT $742 \mathrm{IU} / \mathrm{L}$, Cre $2.68 \mathrm{mg} / \mathrm{dL} 1$ day later.

Results: Her lower extremity compartment pressures were measured on day 3 and were normal. However, an MRI on day 4 showed findings representative of either severe myositis or acute vs. subacute compartment syndrome. 
Discussion: Rhabdomyolysis has been reported in salicylate toxicity, thought to be due to excess neuromuscular rigidity with hyperthermia. Our patient's prolonged hyperthermia prior to arrival may have contributed to her profound rhabdomyolysis. Compartment syndrome can be a complication, and very few case reports involving salicylate toxicity have been reported.

Conclusion: Compartment syndrome should be considered in patients that develop severe pain and swelling in the setting of salicylate toxicity.

\section{Salicylate Toxicity from Ingestion of an Oil of Wintergreen- Containing Insecticide}

\section{Michael Moss}

Utah Poison Control Center, College of Pharmacy, University of Utah, Salt Lake City, Utah, USA. Division of Emergency Medicine, Department of Surgery, University of Utah, Salt Lake City, Utah, USA

Background: Oil of wintergreen is well-recognized as a source of salicylate exposure. However, salicylate toxicity from insecticide ingestion is not reported.

Case report: This is a single-patient chart review. A 74-year-old female weighing $43 \mathrm{~kg}$ reported ingesting two cups of EcoLogic ${ }^{\mathrm{TM}}$ Home Insect Control insecticide in a suicide attempt. She complained of vomiting and abdominal pain. Labs $2 \mathrm{~h}$ after ingestion were salicylate concentration ([ASA]) $72.5 \mathrm{mg} / \mathrm{dL}$, blood gas $\mathrm{pH} 7.44, \mathrm{pCO}_{2} 41$, and serum bicarbonate $26 \mathrm{mmol} / \mathrm{L}$. [ASA] units were verified as $\mathrm{mg} / \mathrm{dL}$. She developed difficulty hearing and was treated with sodium bicarbonate with a steady decline in [ASA]. Symptoms improved, and she was discharged $36 \mathrm{~h}$ later.

Results: Peak [ASA] was $72.5 \mathrm{mg} / \mathrm{dL}$ at $2 \mathrm{~h}$. Subsequent [ASA]: $59 \mathrm{mg}$ / $\mathrm{dL}$ at $6 \mathrm{~h}, 39.3 \mathrm{mg} / \mathrm{dL}$ at $18 \mathrm{~h}, 22.8 \mathrm{mg} / \mathrm{dL}$ at $30 \mathrm{~h}$, and $17 \mathrm{mg} / \mathrm{dL}$ at $38 \mathrm{~h}$. The manufacturer's website indicated $1.5 \%$ lemongrass oil as the active ingredient and an unspecified quantity of other ingredients including wintergreen oil. Further details of product composition were not available from the manufacturer. Assuming 100\% bioavailability, a volume of distribution of $0.15 \mathrm{~L} / \mathrm{kg}$, molecular weight ratio of methyl salicylate to salicylate of 1.11, peak concentration of $72.5 \mathrm{mg} / \mathrm{dL}$, and ingested volume of $16 \mathrm{oz}$, the product may have contained a $1.1 \%$ weight/volume solution of methyl salicylate.

Discussion: This case demonstrates significantly elevated [ASA] from ingestion of a methyl salicylate containing insecticide for home use. The United States Environmental Protection Agency recognizes methyl salicylate as an inert ingredient with no specific labeling required. Limitations include the possibility of ingestion of another source of salicylate and lack of confirmation of methyl salicylate in the ingested product.

Conclusion: Methyl salicylate containing insecticides may cause potentially serious salicylate toxicity. Exposure may go unrecognized as inert ingredients are not necessarily listed on safety data sheets.

046. Hepatotoxicity After Sustained Use of Polygonum Multiflorum Musa S Alfaifi ${ }^{1}$, Waleed Alsukaiti ${ }^{1}$, Ziad Kazzi ${ }^{1}, \mathrm{~K}$ Barada ${ }^{2}, \mathrm{Z}$ Mneimneh $^{2}$, E Hitti $^{2}$, M Malaeb ${ }^{2}$, Melissa Gittinger ${ }^{1}$

${ }^{1}$ Emory University School of Medicine, Atlanta, GA, USA. ${ }^{2}$ American University of Beirut, Beirut, Lebanon

Background: Polygonum multiflorum (PM) is a Chinese herb used as an antioxidant and anti-aging agent. It can cause acute hepatotoxicity, liver failure, and death. There is no published data on the effectiveness of $\mathrm{N}$ Acetyl cysteine (NAC) as sole therapy.

Methods: This is a case report of a 23-year old healthy male who presented to the ED with jaundice for 1 month. The patient reported taking $12 \mathrm{~g} /$ day of the PM, for 2 months to promote longevity. He had no history of recent travel, illicit drug, or alcohol abuse. Physical examination revealed jaundice, with no stigmata of chronic liver disease. Admission labs demonstrated transaminitis (AST $1163 \mathrm{U} / \mathrm{L}$, ALT $3482 \mathrm{U} / \mathrm{L}$ ) and increased total and direct bilirubin. Testing for infectious and autoimmune causes was unremarkable and an abdominal ultrasound was suggestive of hepatitis.
Results: The patient was admitted, and $P M$ was stopped. A loading dose of oral NAC was given, and maintenance oral NAC was continued for 4 days. On the fourth day, the patient was asymptomatic with improving liver enzymes. He was discharged on oral NAC. He returned 2 days later with worsening jaundice and re-elevation of transaminases (AST $1039 \mathrm{U} /$ $\mathrm{L}$ and ALT $3014 \mathrm{U} / \mathrm{L}$ ). The patient denied re-consumption of $P M$ or other hepatotoxins. The supplement was tested by GCMS and measured the presence of emodin (the hepatotoxic component of $P M$ ). He was readmitted, NAC was continued, and his liver enzymes eventually improved.

Discussion: This case highlights the potential benefit of NAC as a treatment of emodin-induced hepatotoxicity. Emodin likely causes hepatotoxicity through depletion of glutathione. NAC's replenishment of intracellular glutathione may, therefore, offer benefit.

Conclusion: Polygonum multiflorum use has been associated with severe and prolonged hepatotoxicity. Oral NAC therapy should be considered in the treatment of these patients due to the favorable risk-benefit ratio.

\section{Sudden Cardiac Death in a Bodybuilder}

Lauren Murphy, Adam Blumenberg, Tony Rianprakaisang, Adrienne Hughes, Robert Hendrickson

Oregon Health and Science University, Portland, OR, USA

Background: The synthetic opioid difenoxin is the active metabolite of diphenoxylate, which is found in anti-diarrheal atropine/diphenoxylate products.

Methods: Single patient case report.

Case: A 30-year-old male bodybuilder developed pulseless ventricular tachycardia that was successfully defibrillated by EMS. On initial evaluation, the patient was tachycardic (HR 119) and hypertensive (BP 150/110) with an unremarkable physical examination and laboratory workup. ECG showed QRS 102, repolarization abnormalities, and QTc 464. Emergent cardiac catheterization, transthoracic echocardiogram, stress testing, and cardiac MRI identified no source of his arrest. He later reported taking the following body-building supplements: clenbuterol $80 \mu \mathrm{g} /$ day, testosterone propionate $600 \mathrm{mg} /$ day, drostanolone propionate $500 \mathrm{mg} /$ week, stonazolol $50 \mathrm{mg} /$ day, anastrozole $2 \mathrm{mg} /$ day, triiodothyronine $25 \mathrm{mg} /$ day, and caffeine. Blood and urine were analyzed using targeted analysis (QTOF-MS) and found to have high concentrations of difenoxin with insignificant concentrations of his supplements, including caffeine, trenbolone, stonazolol, and anastrozole. He was discharged after placement of a defibrillator.

Discussion: Difenoxin is the primary metabolite of diphenoxylate and has caused opioid toxicity and death in children after ingestions of diphenoxylate/atropine products. There are no previous cases of dysrhythmia. Difenoxin and diphenoxylate are congeners of meperidine. Meperidine and opioids such as loperamide and methadone are known to cause ventricular dysrhythmias. Given that difenoxin was found in large quantities in the patient's blood and a thorough search for other causes did not identify other causes, we postulate an association between difenoxin and ventricular tachycardia. Additional cases, as well as information on conduction and receptor effects will be necessary to solidify this association. Our patient denied diphenoxylate use. It is not clear if difenoxin was an adulterant in one of his supplements, which were not all tested.

Conclusion: Clinicians should be aware that difenoxin may be associated with bodybuilding supplements and ventricular dysrhythmias. Additional case evidence will help to refute or confirm this association.

\section{A Pure T3 Thyrotoxicosis Due to Redotex Ingestion, a Mexican Weight Loss Drug}

Han Gao, Alicia Minns

University of California San Diego, San Diego, California, USA

Background: Redotex is a weight loss supplement consisting of five ingredients: tri-iodothyronine $75 \mu \mathrm{g}$, atropine $0.36 \mathrm{mg}$, diazepam $8 \mathrm{mg}$, 
aloin $16 \mathrm{mg}$, and d-norpseudoephedrine $50 \mathrm{mg}$ per tablet. There are few case reports with clinically severe ingestions. Here, we present a chronic ingestion exhibiting major effects.

Methods: A 37-year-old female presented with chest pain and nausea after ingesting two tabs of Redotex. Initial vital signs reflect HR $134 \mathrm{bpm}$ and BP 130/66 mmHg. The patient received 21 of crystalloid and ondansetron IV with some mild relief, however left against medical advice shortly after. She returned the next day with worsening chest pain, palpitations, and tremors. She admitted to taking Redotex 1-2 tabs daily for the last 6 weeks. She was admitted to the ICU for supportive care.

Results: Thyroid function tests on initial presentation showed a TSH $0.013 \mathrm{uU} / \mathrm{mL}$ (normal $0.5-4.7 \mathrm{uU} / \mathrm{mL}$ ), free $\mathrm{T} 40.24 \mathrm{ng} / \mathrm{dL}$ (normal $0.8-1.8 \mathrm{ng} / \mathrm{dL}$ ), and free $\mathrm{T} 3>30 \mathrm{pg} / \mathrm{mL}$ (normal $2.3-4.2 \mathrm{pg} / \mathrm{mL}$ ). Propranolol and benzodiazepines were recommended by the toxicology service, and the patient received one dose of propranolol with symptomatic relief. While in the ICU, the patient was placed on a scheduled propranolol $10 \mathrm{mg}$ IV BID regimen and received 2 doses of lorazepam $1 \mathrm{mg}$ IV for breakthrough chest pain and agitation. Repeat thyroid function studies after $24 \mathrm{~h}$ showed a TSH $<0.007 \mathrm{uU} / \mathrm{mL}$, free T4 $0.18 \mathrm{ng} / \mathrm{dL}$, and free T3 $16.8 \mathrm{pg} / \mathrm{mL}$. Patient is asymptomatic and discharged on hospital day three.

Discussion: When taken chronically, Redotex can cause T3 thyrotoxicosis. This has not been seen in prior reports. In two case series totaling 291 patients, there were no fatalities and only one major outcome where the patient developed a goiter.

Conclusion: Chronic Redotex ingestion can cause clinically significant thyrotoxicosis.

\section{Dimethylamylamine Exposures Reported to a Statewide Poison Control System}

Rais Vohra ${ }^{1,2}$, Serena Huntington ${ }^{1}$

${ }^{1}$ California Poison Control System, Fresno-Madera Division at Valley Children's Hospital, Madera, CA, USA. ${ }^{2}$ UCSF Fresno Medical Education and Research Program, Fresno, CA, USA

Background: Once marketed as a rhinitis treatment, 1,3dimethylamylamine (DMAA) is structurally similar to amphetamines and works as an indirect sympathomimetic agent. Adverse effects include seizures, livery and renal injury, cardiac arrest, and death. In 2013, the US Food and drug Administration (FDA) mandated removal of these products from the market; however, they can still be found online and in sports nutrition stores.

Methods: This is an IRB-exempt, retrospective study of deidentified DMAA exposures reported to a statewide poison control system over a ten-year period. Cases were identified using the search terms "Dimethylamylamine" or "DMAA." Case records containing information-only calls, non-human ingestion, wrong substance, and/or cases with no clinical data were excluded. Descriptive statistics were performed on the abstracted data.

Results: A total of 124 cases were identified. The majority of cases were less than 5 years old (59 cases; $47.6 \%$ ) with an age range of 8 months to 59 years old (median age, 11 years old). The gender distribution was relatively equal (54\% males). Exposure intent varied, with 59 cases (48\%) of unintentional accidental exposure, 17 cases (14\%) of intentional misuse, 8 cases (7\%) of unintentional therapeutic error, and 4 cases (3\%) of intentional self-harm. Outcomes: $45(36 \%)$ cases resulted in minor effects, $6(5 \%)$ resulted in moderate effects, and $1(1 \%)$ resulted in major effects. Significant toxicity was associated with adult bodybuilders, who developed one or more of the following: presyncope, vomiting, diaphoresis, seizure, rhabdomyolysis, jaundice, hepatitis, and supraventricular tachycardia.

Conclusions: Although the exposure trends have been declining over the past few years, exposures to DMAA supplements are still occurring, especially with its popularity in the bodybuilding community. More serious outcomes were associated with cardiac, hepatotoxic, and central nervous system (CNS) effects. Adult exposures in bodybuilders/dieters appear to be more clinically severe than pediatric/accidental exposures.

\section{Serious Adverse Events Following the Use of African Black} Ant-a Dietary Supplement

Kevin Kammel ${ }^{1}$, Ahmed Mamdouh Taha Mostafa ${ }^{2}$, Rita McKeever ${ }^{2}$

${ }^{1}$ Drexel College of Medicine, Philadelphia, PA, USA. ${ }^{2}$ Division of Medical Toxicology, Drexel University College of Medicine, Philadelphia, PA, USA

Background: A 2017 survey reported that $76 \%$ of US adults use dietary supplements. Many "all natural" supplements are adulterated with prescription and over-the-counter medications that have caused adverse events.

Methods: This is a case report of a 50-year-old male with a past medical history of diabetes, hypertension, end-stage renal disease on hemodialysis, polysubstance abuse, paroxysmal atrial fibrillation, and congestive heart failure who presented to the emergency department for altered mental status during hemodialysis. As he was transferred to a room from triage, he became unresponsive. Vital signs initially included pulse 179, BP 58/30 mmHg with an ECG demonstrating atrial fibrillation with rapid ventricular response. He received one synchronized cardioversion at $100 \mathrm{~J}$ with improvement of his blood pressure and was intubated for airway protection. He had a recurrence of the tachyarrhythmia that was refractory to diltiazem and amiodarone but converted with lidocaine into sinus tachycardia with frequent PVCs. He was treated with sodium bicarbonate and insulin/glucose for hyperkalemia $(6.4 \mathrm{mmol} / \mathrm{L})$ and admitted to the intensive care unit. He later required an intra-aortic balloon pump (ejection fraction 15-20\%). His son reported that the patient had been using the sexual enhancement supplement "African Black Ant" (ABA) and had used it the night prior to presentation.

Discussion: ABA is marketed as a natural supplement for improvement of sexual function, but it was found to contain sildenafil. The FDA has issued a public notification about this supplement, warning people of the potential for containing hidden harmful ingredients. Moreover, manufacturers of sexual enhancement supplements have increasingly turned to entirely novel pharmaceutical products that are not easily identified and have no regulatory status. Conclusion: Dietary supplements should be strictly regulated. To our knowledge, this is the first case where a patient suffered life-threatening adverse events following the use of this supplement.

\section{Antimuscarinic Toxicity Secondary to Lupini Bean} Consumption Requiring Physostigmine

Ryan Marino, Ross McCormack, Spiros Giannoutsos, Kenichi Tamama, Joseph Yanta

University of Pittsburgh School of Medicine, Pittsburgh, PA, USA

Background: The lupin or lupini bean, the seed of Lupinus albus, is commonly consumed in various Mediterranean cultures. It contains bitter tasting alkaloids, mainly lupanine, which require intensive preparation to be removed from the edible product. Despite widespread availability, reports of toxicity are exceedingly rare, and the resultant toxidrome is incompletely understood.

Hypothesis: Alkaloids contained in Lupinus albus seeds cause toxicity through antagonism of muscarinic acetylcholine receptors.

Methods: This is a single patient case report. A 47-year-old man with no significant past medical history presented to the Emergency Department with complaint of dry mouth. Vital signs were remarkable only for hypertension (146/96 mmHg). On exam, he was encephalopathic, with visual hallucinations, mydriasis, urinary retention, carphologia, and muffled speech. He was given $2 \mathrm{mg}$ intravenous physostigmine with complete resolution of all symptoms and normalization of blood pressure $(116 / 72 \mathrm{mmHg})$. He later stated he had made a cassoulet using dry, unprepared lupini beans, and noted a strong bitter taste. 
Results: Urine enzyme-multiplied immunoassay technique (EMIT) screen was positive for tetrahydrocannabinol, which he stated he used on occasion, and phencyclidine (PCP). Urine gas chromatography/mass spectrometry (GC/MS) revealed a massive peak of lupanine, a small peak of sparteine, and a smaller peak of caffeine. GC/MS showed no evidence of phencyclidine or other chemicals known to cause false positive PCP urine drug screen, and the pathologist reported this as a false positive result. Subsequent testing of isolated lupanine and sparteine extracts yielded false-positive PCP results. Serum ethanol, acetaminophen, and salicylate were undetectable.

Discussion: Lupanine has been reported to cause a toxidrome with antimuscarinic features. This is the first case of lupanine toxicity resolving with physostigmine administration, confirming that lupanine antagonizes acetylcholine receptors.

Conclusion: Unprepared lupini bean consumption can cause an antimuscarinic toxidrome reversible with physostigmine and false positive PCP immunoassay.

\section{Brodifacoum-laced Synthetic Cannabinoids-Analytical and} Practical Concerns for the Toxicology Laboratory

\section{Robert Middleberg}

NMS Labs, Willow Grove, PA, USA

Background: In the first quarter of 2018, individuals seeking medical care for unexplained bleeding appeared primarily in the Midwest US. Subsequently, additional cases in other geographies were reported.

Methods: Based on clinical signs and laboratory-based hemostasis markers, blood from the sentinel case was screened for anticoagulants using an LC-MS/MS-based analysis.

Results: The blood specimen tested positive for the superwarfarin brodifacoum. An excess of 100 additional submissions from patients with similar clinical signs also tested positive for brodifacoum. Additionally, relatively small amounts of the superwarfarins difenacoum and bromadiolone were detected. A number of deaths occurred as a result of failure to seek treatment or to comply with treatment. The only common history among the patients was recent use of synthetic cannabinoid products. Testing of some synthetic cannabinoid products by law enforcement laboratories confirmed the presence of brodifacoum

Discussion: For the toxicology laboratory, a number of challenges were confronted, including how to ramp up an infrequently requested test to meet this urgent need, how to make it affordable from both a laboratory and user perspective, and how to develop a validated quantitative method in a short period of time. Further, organizing resultant data was challenging due to the disparate agencies submitting specimens, inability to identify readily cohort cases, and dissemination of testing results. This event ultimately solidified the need for coordinated efforts between governmental agencies (both federal and state), law enforcement, treating facilities/clinicians, and the testing laboratory to identify the causative agent, to monitor success of treatment, to define the scope of the problem, and attempts to control it.

\section{The Curious Case of Mistaken Baby Formula Identity:} Organophosphate Toxicity in a Neonate

Sukhshant K Atti ${ }^{1}$, Emily Kiernan ${ }^{1}$, Mark R Layer ${ }^{1}$, Darius Watts ${ }^{1}$, Taryn Taylor $^{2}$, Melissa Gittinger ${ }^{1,3}$

${ }^{1}$ Emory University School of Medicine, Atlanta, GA, USA. ${ }^{2}$ Children's Hospital of Atlanta, Atlanta, GA, USA. ${ }^{3}$ Georgia Poison Center, Atlanta, GA, USA

Background: Ingestion is reported as the most common route of pediatric organophosphate exposure. There are limited reports of organophosphate ingestion in neonates.

Hypothesis: Due to premature physiology, neonates may have severe complications after organophosphate exposure.
Methods: This is a single patient case report. An ex-premature, $4.7 \mathrm{~kg}$ male (born at 35 weeks, with a 2-week NICU stay), 2-week-old by corrected age, with no other medical history, presented to EMS after being fed an organophosphate that was mistaken for formula. He presented with increased secretions, respiratory distress, and diarrhea. During EMS transport, he developed apnea and was given atropine, lorazepam, and was started on supplemental oxygen. In the ED, the patient's initial examination was notable for hypertension (113/68) and tachycardia (212 bpm). He was lethargic, and demonstrated fasciculations, increased secretions, miosis, diarrhea, and increased work of breathing. He was intubated secondary to respiratory failure; $0.01 \mathrm{mg} / \mathrm{kg}$ IV atropine, and $10 \mathrm{mg} / \mathrm{kg}$ IV pralidoxime bolus were initiated, followed by a pralidoxime infusion for $24 \mathrm{~h}$.

Results: During admission, the patient received atropine $0.2 \mathrm{mg}$ IV every $3 \mathrm{~h}$. Hospital course was complicated by continued secretions and apnea. His admission RBC acetylcholinesterase level resulted at $11 \mathrm{U} / \mathrm{g} \mathrm{Hb}$ (ref 31.2-61.3). Atropine was discontinued by the inpatient team after an episode of desaturation secondary to mucus plugging. His secretions recurred and glycopyrrolate was initiated. On day 7 , he was extubated. Ongoing management included glycopyrrolate, dexamethasone, and standard respiratory therapies. Despite resolution of organophosphate toxicity, his hospital course was prolonged by poor feeding and airway edema. He was discharged on day 16.

Conclusion: In organophosphate toxicity, children may present with minimal muscarinic toxicity but with predominantly neurological abnormalities such as seizures, CNS depression, flaccidity, and diaphragmatic failure. Neonates have lower baseline levels of RBC cholinesterase, potentially contributing to prolonged organophosphate toxicity.

\section{Acute poisoning: What kills our patients in ED?}

Ashish Bhalla, Vamshi V, Navneet Sharma, Susheel Kumar, Debaprasad Dhibar, Surjit Singh

Post Graduate Institute of Medical Education and Research, Chandigarh, UT, India

Objective: Acute poisoning is common in India.

Methods: A prospective study was conducted from December 1st, 2016 to November 30th, 2017 in the emergency department of Post Graduate Institute for Medical Education and Research, Chandigarh to evaluate the potential causes of mortality in acute poisoning. All patients included in this study were $>12$ years old with alleged history of acute poison consumption.

Results: A total of 330 cases were screened but only 313 could be included in the study. All the patients underwent clinical evaluation and management according to standard protocols followed in hospital. Among 313 cases, $61 \%$ were males and $38 \%$ were females. Mean age group in this study was $29.6 \pm 13.2$ years. Most poisoning cases were seen in the younger age group (21-40 years) which contributed to $61 \%$ of total cases. Most patients had a rural background (64.9\%). The poisoning was more common in patients with an agricultural background (36.7\%). Twenty-nine percent were students, $15 \%$ were housewives, and $10 \%$ were unemployed. In $98 \%$, the mode of exposure was ingestion. Intake for self-harm was reported in $75 \%$, whereas $25 \%$ claimed exposure to be accidental. In $31 \%$, the poisoning was related to pesticide, $20 \%$ were metal phosphides, $9.6 \%$ were prescription drugs, $7.5 \%$ were corrosives, and only $5 \%$ were opioids. More than $69 \%$ of cases eventually improved and were discharged, $17.3 \%$ left against medical advice, and 14\% died. Mortality was maximum in patients aged 21 to 40 years. Only $10 \%$ of those who died had received any form of first aid, and only $8 \%$ had received antidotes prior to reaching us, but inadequate doses. Delayed presentation, hypercarbia, low GCS, and liver dysfunction were the parameters related to mortality on multivariate logistic regression.

Conclusions: Pesticides remain the commonest cause of poisoning in our set up. The mortality was related to delayed presentation, delayed institution of antidotes or supportive care. 


\section{Day 2: Platforms, Abstracts 055-058}

\section{Circulating microRNA Profiles in Acetaminophen Toxicity}

Stephanie Carreiro, Rosiland Lee, James Marvel-Coen, Brittany Chapman, Victor Ambros

University of Massachusetts Medical School, Worcester, MA, USA

Background: Acetaminophen (APAP) toxicity can be difficult to differentiate from other conditions that cause transaminase elevation. Previous work has described elevation of specific miRNAs in APAP-induced liver injury.

Hypothesis: miRNA profiles can differentiate between cases of confirmed APAP toxicity from transaminase elevations not related to APAP. Methods: This is an observational study of adults presenting to the emergency department with elevated transaminases and initial concern for APAP toxicity. Serum samples were collected every $12 \mathrm{~h}$ during hospitalization. Total miRNAs were extracted from plasma and the levels of 327 microRNAs were quantified using real-time-PCR. A standard measure of miRNA expression (delta-delta-cycle threshold) was calculated for each miRNA, and peak samples for each participant (those that contained the most miRNAs at a level eightfold higher than controls) were identified. Two level cluster analysis was performed using a random k-means algorithm. Demographic and clinical characteristics of each cluster were compared using ANOVA and chi square.

Results: Twenty-seven participants were enrolled (21 confirmed APAP toxicity, 6 non-APAP), and a total of 61 samples were analyzed. Two level clustering of the peak samples yielded five clusters, two of which demonstrated clear clinical patterns. Features associated with cluster 1 $(p<0.05)$ included confirmed APAP toxicity, undetectable APAP level, high peak alanine aminotransferase (ALT), and late presentation. Features associated with cluster $5(p<0.05)$ included confirmed APAP toxicity, high ALT and number of peak miRNAs, detectable initial APAP level, and late presentation. Clusters 2-4 contained lower peak miRNAs, more heterogeneous clinical characteristics, and included all but one of the nonAPAP toxicity participants.

Conclusion: The most severe cases of APAP toxicity showed two distinct patterns of miRNA elevation, while less severe cases were more difficult to distinguish from non-APAP associated cases. Further work is needed to determine the clinical utility of miRNAs in the diagnostic algorithm of APAP toxicity.

056. Macrophage Migration Inhibitory Factor as a Therapeutic Target in Acetaminophen-Induced Liver Injury

Joshua Bloom, Mingzhu He, Yousef Al-Abed

Center for Molecular Innovation, The Feinstein Institute for Medical Research, Manhasset, NY, USA

Background: Macrophage migration inhibitory factor (MIF) is a pleiotropic cytokine and enzyme that has been implicated in multiple inflammatory and non-inflammatory diseases, including liver injury induced by acetaminophen (APAP) overdose. MIF is well known to possess oxidoreductase activity and may promote oxidative stress in the setting of APAP overdose. Methods: This is a preclinical study of the role of MIF and anti-MIF small molecule drugs in the toxicology of acetaminophen-induced liver injury using a well-established murine model. Toxicity was induced by administration of acetaminophen to $\mathrm{C} 57 \mathrm{Bl} / 6$ mice and/or matched MIF knock-out animals, which were treated with the anti-MIF drug iguratimod or placebo as either prophylaxis or therapy. Animals were monitored for survival as well as liver $\mathrm{H}_{2} \mathrm{O}_{2}$, a surrogate marker of oxidative stress. Survival studies used ten subjects per group and were analyzed with a log-rank test. Biomarker studies used four to seven subjects per group and were compared using Student's $t$ test.

Results: APAP intoxication under the conditions of this study was lethal within 5 days. Anti-MIF therapy applied after intoxication did not significantly improve survival over control ( $p=\mathrm{NS})$. However, when given prophylactically, iguratimod significantly improved survival to $50 \%$ at 5 days $(p=0.009)$. Iguratimod also decreased liver $\mathrm{H}_{2} \mathrm{O}_{2}$ levels by $0.1 \mu \mathrm{g} / \mathrm{mg}$ post-toxicity $(p<0.05)$ but failed to do so in animals lacking MIF.

Conclusion: MIF promotes oxidative stress in acetaminophen toxicity, and MIF inhibition can prevent this effect and decrease the toxicity of APAP. Iguratimod may hold promise as a prophylactic adjuvant to acetaminophen.

\section{A Synthetic Cannabinoid Mass-Overdose in New Haven,} Connecticut

Daniel Nogee ${ }^{1}$, Thomas Durant ${ }^{1}$, Dustin Bunch ${ }^{1}$, Barry Logan ${ }^{2}$, Sandy Bogucki ${ }^{1}$, Asim Tarabar ${ }^{1}$, Anthony Tomassoni ${ }^{1}$

${ }^{1}$ Yale New Haven Hospital, New Haven, CT, USA. ${ }^{2}$ Center for Forensic Science Research \& Education, Willow Grove, PA, USA

Background: In August 2018, multiple intoxicated patients presented in rapid sequence to emergency departments (EDs) in New Haven, Connecticut as a result of a new batch of "K2" synthetic cannabinoids (SC) entering the local drug supply chain. This outbreak attracted national media attention and intensive local responses from health and law enforcement agencies.

Methods: In this case-series, electronic medical records of all patients presenting to local EDs during the 6-day outbreak were reviewed to identify patients meeting the case-definition of suspected SC use. Blood and urine samples were analyzed using enzyme-linked immunosorbent assay (ELISA) and liquid chromatography quadrupole time-of-flight mass spectrometry (LC-QTOF). Several patients were interviewed to obtain more information about SC distribution.

Results: Fifty-two patients meeting the case definition presented over 109 ED visits (range 1-13 visits per patient); 49 of these visits occurred within a 10 -h period on August 15th. Symptoms varied significantly between visits (including patients with multiple visits) and included decreased mental status (51/109), agitated delirium (13/109), and seizures $(5 / 109)$. Naloxone use was documented in 13/109 visits, with mixed responses. Two patients were intubated for decreased mental status. Urine samples from 21 patients were found to contain 5F-MDMB-PICA (5/21), 5F-ADB and MMB-FUBINACA metabolites (20/21), and fentanyl (4/21). Unstructured interviews with patients revealed that the $\mathrm{K} 2$ may have been given away freely or very cheaply by multiple dealers targeting methadone users in a competitive open-air market on the town green.

Conclusions: This SC use outbreak was notable for the acute demand it placed on ED and prehospital resources and cost to the healthcare system. Health and law enforcement agency response may have reduced hospital impact by evaluating patients on-site and interrupting supply. SC novelty and accessibility likely encouraged repeated use. K2 products containing multiple SC and co-ingestions may have contributed to the appearance of multiple clinical phenotypes.

\section{Naloxone in the Era of Illicit Fentanyl: What Is the Dose?}

Joseph Carpenter, Brian Murray, Sukhshant K Atti, Arthur Yancey, Tim Moran, Brent Morgan

Emory University School of Medicine, Atlanta, GA, USA

Background: Fentanyl and fentanyl analogues are widespread within the illicit drug supply. Small case series have suggested that fentanyl-related overdoses may require larger doses of naloxone than a heroin overdose, however this finding was not replicated in a larger cohort study. We aimed to determine if the naloxone dose required to treat an opioid overdose is associated with the finding of fentanyl, opiates, or both on urine drug screen (UDS).

Methods: This was a retrospective chart review of opioid overdose patients treated in a single emergency department (ED) between 1/1/2017 
and $6 / 15 / 2018$. We reviewed the charts of all patients who received naloxone from emergency medical services (EMS) during the study period. Adults suffering a non-suicidal opioid overdose were included if they had UDS performed in the ED that was positive for fentanyl, opiates, or both. Data collected included demographics, vital signs, EMS and ED treatments, laboratory findings, and ED disposition. The fentanyl-only and fentanyl + opiate groups were compared to the opiate-only group using the stratified (by provider) variant of the Mann-Whitney $U$ test.

Results: We reviewed 837 charts and identified 130 subjects meeting inclusion criteria. The median age was 38 years (range 18-77); 77\% of subjects were male. The median initial GCS was 3 (IQR: 3-8) and median initial respiratory rate was 6 (IQR: 4-10). Thirty-one subjects' UDS were positive for opiates, 24 for fentanyl, and 75 were positive for both opiates and fentanyl. In the naloxone dose analysis, neither the fentanyl-only (median $0.8 \mathrm{mg}$, IQR $0.4-1.6 ; p=0.68$ ) nor the fentanyl + opiate (median $0.8 \mathrm{mg}$, IQR $0.4-1.2 ; p=0.56$ ) groups differed from the opiate-only group (median $0.58 \mathrm{mg}$, IQR 0.4-1.6).

Conclusion: In this group of opioid overdose patients, there was no significant association between the required naloxone dose and UDS results.

\section{Day 2: Moderated Posters, Abstracts 59-64}

059. Serum ALT Activity at Commencement of $\mathrm{N}$-acetylcysteine Therapy as a Predictor of Hepatotoxicity, Hepatic Encephalopathy, and Mortality in Patients with Acetaminophen Toxicity

Steven Curry ${ }^{1,2}$, Angela Padilla-Jones ${ }^{1,2}$, A. Kang ${ }^{3,2}$, Jerry Snow ${ }^{1,2}$, C. Heise $^{1,2}$

${ }^{1}$ Department of Medicine, Divisions of Clinical Data Analytics and Decision Support, and Medical Toxicology and Precision Medicine, University of Arizona College of Medicine - Phoenix, Phoenix, Arizona, USA. ${ }^{2}$ Department of Medical Toxicology, Banner University Medical Center Phoenix, Phoenix, Arizona, USA. ${ }^{3}$ Department of Child Health; Department of Medicine, Divisions of Clinical Data Analytics and Decision Support, and Medical Toxicology and Precision Medicine, University of Arizona College of Medicine Phoenix, Phoenix, Arizona, USA

Background: $N$-acetylcysteine (NAC) prevents liver failure if begun early after APAP overdose. However, ingestion histories are commonly unreliable, and patients may show normal liver function tests for about $24 \mathrm{~h}$ before clinical evidence of liver injury appears.

Research Question: Does ALT value at time of commencing NAC assist in predicting outcomes?

Methods: 500 subjects $\geq 18$ years of age with suspected APAP overdose were enrolled in a prospective observational study. Subjects categorized at discharge as having definitely overdosed on APAP and for whom ALT was measured \pm 4 h of beginning NAC ("initial" ALT) were included for analyses. Hepatotoxicity $=$ peak ALT $>1000$. Positive and negative predictive values (NPV and PPV) and confidence intervals (CI) were calculated. This study was approved by Banner Health IRB.

Results: Three hundred eighteen subjects met inclusion criteria. Ten of 160 subjects with initial ALT $\leq 50 \mathrm{IU} / \mathrm{L}$ developed hepatotoxicity $(\mathrm{PPV}=$ $.06 ; 95 \%$ CI .03 to .11), 1 developed hepatic encephalopathy (HE) (PPV $<.01 ; 95 \%$ CI 0 to .03), and none died. Among 191 subjects with initial $\mathrm{ALT} \leq 100 \mathrm{IU} / \mathrm{L}$, a single case of $\mathrm{HE}(\mathrm{NPV}=.99 ; 95 \% \mathrm{CI} .97$ to .99$)$ developed and there were no APAP deaths (NPV $=1.0 ; 95 \%$ CI .98 to 1.0). In contrast, among 127 subjects with initial ALT $>100 \mathrm{IU} / \mathrm{L}, 29$ developed HE (PPV $=.23 ; 95 \%$ CI .15 to .31$)$ and 12 died from APAP (PPV $=.09 ; 95 \%$ CI .05 to .16).

Conclusion: ALT activity at commencement of NAC therapy predicts risk of hepatotoxicity, $\mathrm{HE}$, and death. About $10 \%$ of subjects who present with initial ALT > $100 \mathrm{IU} / \mathrm{L}$ will die from liver failure. This study was performed at a tertiary toxicology treatment and liver transplant center, and results may lack external validity in a different patient population because of referral bias.
060. Acetylcysteine for Acetaminophen Repeated Supratherapeutic Ingestion: How Much Is Needed?

Ron Kirschner ${ }^{1,2}$, Megan Hansen ${ }^{1}$, Miranda Mesloh ${ }^{1}$, Karen Smith ${ }^{2}$, Marcia Rasmussen ${ }^{2}$, Lynette Smith ${ }^{1}$

${ }^{1}$ University of Nebraska Medical Center, Omaha, NE, USA. ${ }^{2}$ Nebraska Regional Poison Center, Omaha, NE, USA

Background: The literature provides less guidance on managing acetaminophen repeated supratherapeutic ingestion (RSTI) compared with acute overdose. This study surveyed US poison center (PC) directors on their recommendations for managing acetaminophen RSTI.

Hypothesis: Some PC directors may consider recommending shorter treatment courses in selected acetaminophen RSTI patients.

Methods: Using the American Association of Poison Control Centers' listserv, an electronic survey link was sent to managing/medical/associate/ assistant directors $(n=379)$ at all 55 US PCs. This study was approved by our institutional review board. The survey asked PC directors about their approach to acetaminophen RSTI in contrast to acute overdose, as well as factors influencing their treatment recommendations.

Results: We received 41 responses from 32 of 55 US PCs, yielding a response rate of $10.8 \%$ for individuals and $58.2 \%$ for PCs. Then, $27 / 41$ $(65.8 \%)$ respondents reported having a guideline that specifically addresses RSTI. When asked about the proportion of acetaminophen cases they estimate to be RSTI-related, respondents' estimates ranged from 5\% to $64 \%$ (mean 23\%). Regarding minimum treatment recommendations in the absence of liver injury, $8 / 41(19.5 \%)$ respondents indicated that $12 \mathrm{~h}$ would be acceptable, while 18/41 (43.9\%) chose 20 or $21 \mathrm{~h}$, and the remainder $(36.6 \%)$ indicated that cessation of treatment would depend on repeat lab results. When asked the proportion of RSTI patients consulted on through their center that develop hepatotoxicity (ALT > $1000 \mathrm{IU} / \mathrm{L}$ ), respondents gave estimates ranging from 1 to $75 \%$ with a mean of $19 \%$. Of the 40 respondents who answered the question on their role at the PC, $75 \%$ were physician toxicologists.

Conclusion: Most PC directors follow a patient-tailored approach to RSTI, with acetylcysteine treatment recommendations guided by acetaminophen clearance and improving aminotransferases. A sizable minority expressed interest in shorter $(12 \mathrm{~h})$ treatment for patients without evidence of liver injury.

\section{Kratom (Mitragyna Speciosa) Exposures Reported to US Poison Centers, 2010-2018}

Joseph Carpenter ${ }^{1,2}$, Royal Law ${ }^{1}$, Stephanie Kieszak ${ }^{1}$, Joshua Schier ${ }^{1}$ ${ }^{1}$ Centers For Disease Control and Prevention, Atlanta, GA, USA. ${ }^{2}$ Emory University School of Medicine, Atlanta, GA, USA

Background: Kratom is an herbal preparation derived from the leaves of Mitragyna speciosa. It has both stimulant and opioid-like effects when consumed, and is used for various reasons including pain relief, increased energy enhancement, and opioid withdrawal self-treatment. It has no FDA-approved indication; however, it is commonly sold online as a dietary supplement. Kratom exposures reported to United States (U.S.) poison control centers (PCCs) increased tenfold from 2010 to 2015; however, more recent data are not available. Our objective was to characterize kratom exposures reported to U.S. PCCs from 2010 to 2018.

Methods: We queried the national PCC reporting database for kratom exposure calls during the specified time period and calculated descriptive statistics. Exposure counts were estimated per million population using U.S. Census Bureau estimates, and trends in annual exposure-related call volume were analyzed using Poisson regression. We assessed the effect of age, single vs. multi-agent exposure, caller location, suicidal intent, and sex on outcome severity using a multivariate logistic regression model. Results: We identified 2483 kratom exposures meeting inclusion criteria. The median age was 30 years (range 1 day-90 years) and $1735(70 \%)$ were male. Two hundred sixty-two (10.6\%) exposures resulted in a severe 
outcome, including 17 deaths, 3 of which were single-agent exposures. The most common clinical effects were tachycardia, nausea, and drowsiness. The most common treatments were intravenous fluids, benzodiazepines, and naloxone. The most common co-exposures were sedativehypnotics, ethanol, and opioids. Annual exposures increased from 0.08 per million in 2010 to 2.13 per million in $2017(p<0.0001)$. Severe outcomes were associated with increasing age $(p=0.02)$, multi-agent exposures $(p<0.0001)$, and exposures reported by a healthcare facility $(p=0.004)$ but not suicidal intent $(p=0.82)$ or sex $(p=0.72)$.

Conclusion: Kratom exposures in the USA continue to increase, representing a potential emerging public health threat. Deaths have occurred, even with single agent exposures.

062. Do No Harm? Hypoglycemic Episodes in Patients Treated under a Hyperkalemia Protocol using IV Regular Insulin

Gerald Maloney, Louis Stokes

Cleveland VA Medical Center, Cleveland, OH, USA. Case Western Reserve University, Cleveland, OH, USA

Background: Hyperkalemia is a common, and potentially life-threatening, electrolyte emergency. To assist in the prompt management of this condition, an order set was built in to our EHR starting July 1, 2016. However, no specific safety evaluation of the order set was done after implementation.

Methods: All charts of patients who received treatment from the hyperkalemia order set from October 1, 2016 to October 1, 2017 were reviewed. The order set included $30 \mathrm{~g}$ of sodium polystyrene sulfate (SPS) orally (unless NPO), 10 units of regular insulin IV followed by $50 \mathrm{~g}$ of $50 \%$ dextrose IV, and $10 \mathrm{ml}$ of $10 \%$ calcium chloride IV if EKG changes. Charts were abstracted for age, renal function, potassium level, and any adverse events related to their treatment. Chart review was performed by the primary author.

Results: One hundred eleven charts met criteria and were reviewed. Then, 39/111 received all 3 medications in the protocol. Further, 98/111 received both insulin and SPS, and 13/111 received SPS only. Then, 81/111 had CKD, with mean SCr of 1.6, with 38/111 being on hemodialysis. Mean pretreatment potassium was 6.1 (range, 5.4-8.1). Further, 24/111 had an adverse event related to hyperkalemia therapy; 23 patients had symptomatic hypoglycemia, and 1 had vomiting and aspiration of the SPS. The hypoglycemic patients had a median nadir glucose of $41 \mathrm{mg} / \mathrm{dL}$, with 6/ 23 dropping to a level $<30 \mathrm{mg} / \mathrm{dL}$. All required at least one bolus of IV dextrose, and two required ICU observation for prolonged hypoglycemia. No events related to use of IV calcium chloride were detected.

Conclusions: Adverse events related to use of a hyperkalemia protocol were not uncommon, with $20.7 \%$ developing significant hypoglycemia. Review of the protocol, specifically the insulin dosing in a population with a high incidence of renal failure, should be undertaken to help reduce further adverse events.

063. Five Patients with Refractory Shock Secondary to Metformin Toxicity Successfully Treated with Intermittent Hemodialysis

Alexander Sidlak, Joshua Shulman, Michael Abesamis, Ryan Marino University of Pittsburgh School of Medicine, Pittsburgh, PA, USA

Background: Metformin-associated metabolic acidosis can lead to profound shock. Toxicity results from metformin's inhibition of complex I of the electron transport chain and carries a high mortality (26-50\%). Reports show better clearance and outcomes with intermittent hemodialysis (iHD) than with continuous veno-venous hemofiltration or hemodiafiltration (CVVHF/HD). Nevertheless, CVVHF/HD has been proposed as an alternative in critically ill patients with the suggestion that iHD use is limited by hypotension.

Hypothesis: Hemodialysis can be therapeutically used in severe metformin toxicity despite refractory shock.
Methods: This is a retrospective chart review of five patients in a single institution over 6 years. Patients with metformin toxicity requiring high-dose vasopressor support and treated with iHD were included. Differences between mean arterial pressure (MAP) prior to, during, and at completion of dialysis were calculated using paired $t$ tests.

Results: Despite critical mean peak lactate $(24.3 \mathrm{mmol} / \mathrm{L}$ [1727.9]), $\mathrm{pH}$ (6.90 [6.78-6.98]), and metformin levels [25-58 $\mu \mathrm{g}$ / $\mathrm{mL}$ ], all five patients recovered and were discharged. The lowest MAP before and during HD were 44 vs. $51 \mathrm{mmHg}(p=\mathrm{NS})$. Two patients required four vasopressors during hemodialysis and all required prolonged iHD (mean $14 \mathrm{~h}$ ). At completion of HD, hemodynamics had improved (51 vs. $82 \mathrm{mmHg} ; p=.0066)$ and vasopressor support decreased.

Discussion: Despite beliefs that this population is "too unstable for HD," we document extended iHD performed on patients in profound shock. Aggressive hemodynamic support, including push-dose vasopressors in one case, temporized vasoplegia, allowing for clearance of metformin and ultimate recovery. With patients on the brink of hemodynamic collapse, the fastest possible clearance of metformin is necessary in order to prevent continued tissue toxicity.

Conclusion: The higher flow rates of iHD are possible in metformin toxicity despite refractory shock and vasopressor support. Treatment by this modality led to good outcomes in this critical population.

064. Evaluating the Need for Digoxin Immune Fab (0vine) Therapy in Chronic Digoxin Toxicity

Nicholas LaFountain, Ryan Marino, Michael Abesamis

University of Pittsburgh School of Medicine, Pittsburgh, PA, USA

Background: Antidotal use of Digoxin Immune Fab has demonstrated clinical utility in acute digoxin toxicity. Evidence-based guidelines, however, do not exist regarding the use of this therapy for chronic digoxin toxicity.

Hypothesis: Treatment of chronic digoxin toxicity with supportive care alone is associated with good outcomes.

Methods: This is a retrospective chart review of adults diagnosed with chronic digoxin toxicity by a medical toxicologist in a hospital system during 01/01/2008-04/30/2018. Diagnosis was based on confirmed chronic home digoxin therapy and serum level $>2.0 \mathrm{ng} / \mathrm{mL}$ at presentation; acutely toxic patients were excluded. Data collected included demographic information, interventions, hemodynamics, laboratory values, and outcomes such as death and length of hospitalization. Descriptive statistical analysis compared the data between subjects who did or did not receive Digoxin Immune Fab.

Results: Nineteen total patients met criteria, 4 of which received Digoxin Immune Fab. Patients who did not receive antidote $(n=15)$ had a $93.3 \%$ survival with the only death from respiratory failure more than $48 \mathrm{~h}$ after normalization of the digoxin level. Then, $66 \%$ presented with hyperkalemia (potassium $>5.0 \mathrm{mmol} / \mathrm{L}$, median $5.9 \mathrm{mmol} / \mathrm{L}$ ), $87.5 \%$ were bradycardic (HR $<60 \mathrm{bpm}$, median $46 \mathrm{bpm}$ ), $60 \%$ were hypotensive ( $\mathrm{SBP}<90 \mathrm{mmHg}$, median $88 \mathrm{mmHg}$ ), with median digoxin level of $3.7 \mathrm{ng} / \mathrm{mL}$. In the group that received antidote $(n=4)$, none had unstable dysrhythmias, although one patient was found with pulseless electrical activity and died shortly after Digoxin Immune Fab administration; other characteristics were similar.

Discussion: Patients treated for chronic digoxin toxicity without antidote administration had favorable outcomes, despite concomitant hyperkalemia, bradycardia, and hypotension. Too few patients were treated with antidote to suggest an associated outcome, despite one observed death, and additional investigation into the safety of Digoxin Immune Fab in chronic toxicity is necessary.

Conclusion: Treatment of chronic digoxin toxicity with supportive care and without use of Digoxin Immune Fab is associated with good outcomes. 


\section{Day 2: Posters, Abstracts 065-107}

065. Repeated Supratherapeutic Acetaminophen Ingestions Managed at a Regional Poison Center

Ron Kirschner ${ }^{1,2}$, Megan Hansen ${ }^{1}$, Miranda Mesloh ${ }^{1}$, Lynette Smith ${ }^{1}$, Marcia Rasmussen ${ }^{1,2}$, Karen Smith ${ }^{2}$

${ }^{1}$ University of Nebraska Medical Center, Omaha, NE, USA. ${ }^{2}$ Nebraska Regional Poison Center, Omaha, NE, USA

Background: Acute acetaminophen (APAP) overdose is well studied; fewer publications address management of repeated supratherapeutic ingestion (RSTI). This study reviewed RSTI cases followed at a regional poison center (RPC).

Hypothesis: A subset of RSTI patients may be appropriate for shortened acetylcysteine treatment.

Methods: The RPC database was searched from 1/2000 to 2/2018 for adult patients admitted to a hospital, treated with acetylcysteine, with at least two ALT determinations. Cases of acute overdose were excluded. Those with undetectable APAP were eligible for inclusion so long as a history of RSTI ( $>4 \mathrm{~g}$ APAP/24 h) was documented. We recorded development of hepatotoxicity (ALT > $1000 \mathrm{IU} / \mathrm{L})$ or liver injury (ALT > $100 \mathrm{IU} / \mathrm{L}$ ), and medical outcome (recovery/death/transplant/ undetermined).

Results: Two hundred sixty-five patients were included for analysis. Age range was 19-90 years (median 43.0). Then, 169 (63.8\%) were female. Further, 67/265 patients (25.3\%) developed hepatotoxicity, of whom $74.6 \%$ had ALT $>1000$ at presentation. In cases where the initial ALT was $<100(n=119)$, only three $(2.5 \%)$ subsequently developed hepatotoxicity (one fatality, initial ALT 69; one transferred to a transplant center, initial ALT 72; one recovery, initial ALT 83). All three presented with detectable serum APAP $(217,149$, and $14 \mu \mathrm{g} / \mathrm{mL}$, respectively). Then, $111 / 265(41.9 \%)$ patients presented with a normal ALT and did not develop liver injury. Regarding outcome, 245/265 (92.5\%) either improved or ALT remained normal, 10 (3.8\%) died, 6 (2.3\%) were transplanted or transferred out of state to a transplant center, and in $4(1.5 \%)$ cases outcome was unknown.

Conclusion: In this review, the outcome of APAP RSTI patients followed a bimodal pattern. About $25 \%$ presented with or developed hepatotoxicity, while over $40 \%$ did not develop liver injury. RSTI patients treated with acetylcysteine who have undetectable APAP and maintain a flat ALT may be appropriate for abbreviated treatment, but prospective studies are needed to determine the optimal timing.

066. Near Miss: Altered Pharmacokinetics after AcetaminophenDiphenhydramine Overdose Leading to Premature Acetylcysteine Cessation with Subsequent Resumption and Patient Recovery

Ron Kirschner ${ }^{1,2}$, Jean Hammack ${ }^{2}$, Margaret Ramirez ${ }^{1}$, Karen Smith ${ }^{2}$ ${ }^{1}$ University of Nebraska Medical Center, Omaha, NE, USA. ${ }^{2}$ Nebraska Regional Poison Center, Omaha, NE, USA

Background: After acetaminophen overdose, common criteria for stopping acetylcysteine treatment include serum concentration $<10 \mu \mathrm{g} / \mathrm{mL}$, stable aminotransferases and international normalized ratio (INR), resolution of encephalopathy, and overall clinical improvement.

Hypothesis: With anticholinergic coingestants, delayed acetaminophen absorption may lead to a second peak after an initial decline, complicating treatment.

Methods: This is a single patient chart review. A 47-year-old woman with a history of alcohol abuse arrived at the ED after an acute intentional self-harm acetaminophen-diphenhydramine ingestion and was intubated for airway protection. Initial labs included acetaminophen $197 \mu \mathrm{g} / \mathrm{mL}$, ethanol $496 \mathrm{mg} / \mathrm{dL}$, AST $32 \mathrm{IU} / \mathrm{L}$, ALT $14 \mathrm{IU} / \mathrm{L}$, INR 1.0. The patient was started on acetylcysteine and admitted to the ICU. After $21 \mathrm{~h}$ of acetylcysteine, acetaminophen was $<10$ and aminotransferases remained normal. The patient was extubated, and acetylcysteine was discontinued. However, on hospital day 3, AST had increased to 312 and ALT to 143. At this point, the patient was noted to be more confused - acetaminophen was repeated and found to be 23 . Providers felt that she was being closely monitored and could not have re-ingested acetaminophen-containing products. The patient was transferred to a transplant center (although she was not a transplant candidate) and acetylcysteine was resumed. AST, ALT, and INR peaked at 19,005 IU/L, $8022 \mathrm{IU} / \mathrm{L}$, and 5.2 , respectively on day 4 and the patient became encephalopathic. Acetaminophen rapidly cleared, though acetylcysteine was continued for 4 days. The patient recovered and was transferred to a behavioral health unit on day 14 of her admission.

Discussion: Altered kinetics have previously been reported with acetaminophen combination products; this case is unusual because the second peak occurred after acetaminophen had become undetectable and acetylcysteine had been stopped.

Conclusion: Clinical decline or worsening liver injury after combination product overdose may suggest a second peak even if acetaminophen has seemingly cleared.

\section{Unintentional Intravenous Administration of Oral Acetaminophen Elixir in an Adult}

Vincent Lee ${ }^{1}$, Benjamin Kessler $^{2}$, Nima Majlesi ${ }^{2}$

${ }^{1}$ Northwell Health, Manhasset, New York, USA. ${ }^{2}$ Staten Island University Hospital, Staten Island, New York, USA

Background: Inadvertent intravenous administration of oral medications is a rarely reported medication error. There are relatively few case reports describing the wrong-route administration of oral elixir acetaminophen given intravenously.

Hypothesis: Intravenous administration of oral elixir acetaminophen has the potential for serious complications.

Methods: This is a single patient chart review. A 76-year-old male with a history of liver cirrhosis, tracheostomy, and gastric adenocarcinoma status-post gastro-jejunal tube was unintentionally administered a total of $256 \mathrm{mg}$ of oral liquid elixir acetaminophen (components: sodium, artificial flavor, citric acid, anhydrous, D\&C Red\#33, FD\&C Red \#40, glycerin, polyethylene glycol 1450, propylene glycol, purified water, saccharin sodium, sodium benzoate, sorbitol) through a left-brachial vein midline catheter. There were no immediate signs of thrombophlebitis or local erythema post-administration. The patient denied any difficulty breathing, chest pain, nausea/vomiting, or left-upper extremity pain. The midline catheter was removed due to the non-sterile preparation.

Results: Complete blood count, metabolic panel, and arterial blood gas were unchanged from the patient's baseline. A 2-h acetaminophen level was undetectable. At $12 \mathrm{~h}$ post-administration, the patient had an episode of difficulty breathing associated with hypotension. A computed-tomography angiography of the chest was performed and ruled-out a pulmonary embolism. Later, this episode was determined to be due to a mucous plug in his tracheostomy. His hypotension resolved with intravenous fluids and proper oxygenation. During the hospital course, the patient did not develop any fevers or signs/symptoms of an upper extremity peripheral vein thrombosis.

Conclusion: Although our patient had no major complications from the unintentional intravenous administration of oral elixir acetaminophen, the viscosity, non-sterile preparation, and inert ingredients could potentially be harmful (i.e., peripheral venous thrombosis, pulmonary embolism, osmotic complications, thrombophlebitis, systemic infection). We recommend removal of the catheter used to prevent potential infectious complications.

068. A Case of Fatal Acetaminophen Ingestion Associated with Methemoglobinemia

Tony Rianprakaisang ${ }^{1,2}$, Adrienne Hughes ${ }^{1,2}$, Adam Blumenberg ${ }^{1,2}$, Lauren Murphy ${ }^{1,2}$, Robert Hendrickson ${ }^{1,2}$

${ }^{1}$ Oregon Health and Science University, Portland, OR. ${ }^{2}$ Oregon Poison Center, Portland, OR 
Background: Acetaminophen is an oxidant that rarely causes methemoglobinemia.

Methods: We present a fatal acetaminophen ingestion associated with methemoglobinemia with serum analysis to exclude other causes.

Case: A 54-year-old cognitively impaired male presented from a group home for AMS. Initial evaluation noted metabolic acidosis, normal brain $\mathrm{CT}$ and MRI. He was admitted to the ICU for management of hypotension and AMS. On day 2, he developed methemoglobinemia (32\%) and received $2 \mathrm{mg} / \mathrm{kg}$ methylene blue. The patient's facility reported no access to medications or history of G6PD deficiency. A 24-h acetaminophen concentration was $531 \mu \mathrm{g} / \mathrm{mL}$ without history of administration in hospital. Repeat bloodwork showed acidemia ( $\mathrm{pH}$ 7.2), hyperlactatemia $(14.5 \mathrm{mmol} / \mathrm{L})$, and elevated aminotransferases (>200 IU/L). NAC and hemodialysis were recommended. He was placed on CRRT and received only the bolus NAC dose due to an error. NAC was restarted after $9 \mathrm{~h}$ with a bolus and $25 \mathrm{mg} / \mathrm{kg} / \mathrm{h}$ infusion. Methemoglobin (14.2\%), acetaminophen concentration $(220 \mu \mathrm{g} / \mathrm{mL})$, and venous $\mathrm{pH}$ (7.29) improved; however, aminotransferases continued to rise (AST $562 \mathrm{IU} / \mathrm{L}$, ALT $407 \mathrm{IU} / \mathrm{L}$ ) and peaked at AST $3487 \mathrm{U} / \mathrm{L}$ and ALT $2837 \mathrm{IU} / \mathrm{L}$. He developed hyperlactatemia $(15.2 \mathrm{mmol} / \mathrm{L})$, coagulopathy (INR 9.8) and required increasing vasopressor support. The family elected to limit interventions and the patient developed PEA arrest and died. Postmortem blood and urine analysis by GC/MS for over 250 common therapeutics revealed only metoprolol and hydroxyzine, both home medications.

Discussion: Acetaminophen is an electrophile and oxidant that is structurally similar to aniline. It is a well-known cause of methemoglobinemia in animals but is rarely reported in humans. This case adds to the literature a case of acetaminophen-induced methemoglobinemia where other potential methemoglobin-causing substances were not detected.

Conclusion: Acetaminophen ingestions may rarely be associated with methemoglobinemia.

\section{Serum and Urine Detection of Phenibut: a Pharmacokinetic Analysis}

Tony Rianprakaisang ${ }^{1,2}$, Michael Moss ${ }^{3,4}$, Adam Blumenberg ${ }^{1,2}$, Adrienne Hughes ${ }^{1,2}$, Lauren Murphy ${ }^{1,2}$, Robert Hendrickson ${ }^{1,2}$

${ }^{1}$ Oregon Health and Science University, Portland, OR. ${ }^{2}$ Oregon Poison Center, Portland, OR. ${ }^{3}$ Utah Poison Control Center, College of Pharmacy, University of Utah, Salt Lake City, UT. ${ }^{4}$ Division of Emergency Medicine, Department of Surgery, University of Utah, Salt Lake City, UT

Background: Phenibut (beta-phenyl-gamma-aminobutyric acid) is a sedative-hypnotic GABA-B agonist structurally similar to baclofen. It is known to cause sedation and seizures in overdose. Mitragynine is the principal alkaloid in kratom, and a weak opioid agonist commonly used in the USA. Pharmacokinetic data on phenibut is limited.

Methods: We report a case of phenibut and mitragynine exposure with serial serum concentrations.

Case: A 38-year-old man with a history of alcohol use, diabetes, gastroparesis, and kratom use presented to an emergency department after three witnessed seizure events. He was encephalopathic and agitated requiring sedation with lorazepam. He was admitted to the ICU for persistent symptoms and subsequently intubated and sedated with propofol. Initial workup including blood work, CT, and MRI/MRA brain were unrevealing. The patient was extubated after 4 days and returned to baseline at which time he admitted to taking a white powder he had obtained from a friend labeled 'phenibut.' Biologic samples (blood and urine) were analyzed using QTOF-MS. Initial serum sample was collected on day 1 and contained $5397 \mathrm{ng} / \mathrm{mL}$ phenibut and $66.6 \mathrm{ng} / \mathrm{mL}$ mitragynine. A second serum sample taken $1421 \mathrm{~min}$ later contained $740 \mathrm{ng} / \mathrm{mL}$ phenibut and $36.5 \mathrm{ng} / \mathrm{mL}$ mitragynine. A final serum sample was taken $1352 \mathrm{~min}$ after the second sample and contained $201 \mathrm{ng} / \mathrm{mL}$ phenibut and $149.37 \mathrm{ng} / \mathrm{mL}$ mitragynine. Serum half-life of mitragynine was $45 \mathrm{~h}$. Serum half-life of phenibut was $9.7 \mathrm{~h}$, and kinetics were first-order. One urine sample was obtained on day 1 and contained $1669 \mu \mathrm{g} / \mathrm{mL}$ phenibut (serum contained $5397 \mathrm{ng} / \mathrm{mL}$ on day 1) indicating that phenibut is concentrated in the urine. Urine also contained $149.37 \mathrm{ng} / \mathrm{mL}$ mitragynine.

Conclusion: We report a case of phenibut and mitragynine associated seizures and agitation. This case provides valuable pharmacokinetic data regarding the elimination and half-life of phenibut, and also indicates that phenibut is concentrated in the urine.

\section{Predictors of Poisoning Severity in Diphenhydramine Overdose}

Adrienne Hughes ${ }^{1}$, Adam Blumenberg ${ }^{2}$, Lauren Murphy ${ }^{2}$, Tony Rianprakaisang ${ }^{3}$, Robert Hendrickson ${ }^{2,4}$

${ }^{1}$ Oregon Health and science University, Portland, OR, USA. ${ }^{2}$ Oregon Health and Science University, Portland, Oregon, USA. ${ }^{3}$ Oregon Health and Science University, Portland, OR, USA. ${ }^{4}$ Oregon Poison Center, Portland, OR, USA

Background: Diphenhydramine (DPH) is a commonly used medication in OTC sleep aids and allergy formulations. DPH is frequently misused recreationally for its antihistaminergic and antimuscarinic effects and is involved in serious and fatal poisonings.

Objective: We describe the signs and symptoms (e.g., delirium/toxic psychosis, agitation, QRS/QT prolongation) associated with severe outcomes (e.g., ventricular dysrhythmias, intubation) in DPH overdoses using the ToxIC registry. Methods: Retrospective review of the ToxIC registry database (2010 2016). Descriptive analysis of all cases with diphenhydramine as the "primary agent" contributing to toxicity. Cases were excluded if there was any other primary agent.

Results: We identified 2465 cases with 864 cases remaining after exclusion criteria (female, 511/864; 59\%). Clinical effects included delirium/toxic psychosis $(n=346,42.1 \%)$, agitation $(n=286,33.1 \%)$, tachycardia $(n=$ $258,29.8 \%)$, hallucinations $(n=179,20.7 \%)$, coma/CNS depression $(n=$ $151,17.4 \%)$, seizures $(n=98,11.3 \%)$, prolonged QTc $(>500 \mathrm{~ms})(n=60$, $7 \%)$, and hyperreflexia/myoclonus/clonus/tremor $(n=40,4 \%)$. There were three deaths $(0.3 \%)$. Seizures were significantly associated with QRS widening (OR 4.83; 95\% CI 1.85-12.58) and QTc prolongation (OR 1.99; 95\% CI 1.04-3.81). Intubation was significantly associated with coma/CNS depression (OR 10.25, 95\% CI 5.99-17.54), QRS widening (OR 15.65, 95\% CI 6.11-40.09), and QTc prolongation (OR 3.05, 95\% CI 1.5-6.21). Ventricular dysrhythmias were significantly associated with QRS widening (OR 37.28, 95\% CI 9.52-145.9) and QTc prolongation (OR 9.5, 95\% CI 2.6-34.6). Seizures, ventricular dysrhythmias, and intubation were not significantly associated with delirium/toxic psychosis and agitation.

Discussion: Severe DPH toxicity, manifesting as seizures, ventricular dysrhythmias, and need for intubation were all significantly associated with cardiac conduction disturbances (QRS/QTc prolongation) but not with delirium/toxic psychosis and agitation. Future studies should be aimed at prospective evaluation of chronology of symptom onset to better predict poisoning severity in DPH overdose and determine if early QRS/QTc prolongation can predict severe toxicity as it does with tricyclic antidepressants.

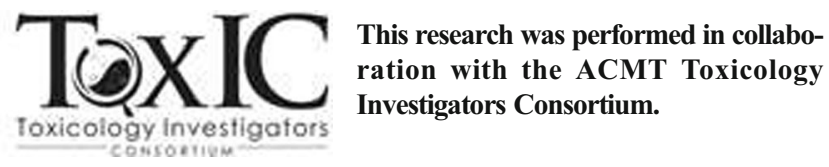

\section{Increasing Misuse and Abuse of Gabapentin and Pregabalin}

Maryann Mazer-Amirshahi ${ }^{1}$, Anne Riederer ${ }^{2}$, Sharan Campleman ${ }^{3}$, Diane Calello ${ }^{4}$, Keith Burkhart ${ }^{5}$, Jeffrey Brent ${ }^{6}$, Paul Wax ${ }^{3}$

${ }^{1}$ MedStar Washington Hospital Center, Washington, DC, USA. ${ }^{2}$ ACMT, Phoenix, AZ, USA. ${ }^{3}$ ACMT, Phoenix , AZ, USA. ${ }^{4}$ Rutgers, Newark, NJ, USA. ${ }^{5}$ FDA, Silver Spring, MD. ${ }^{6}$ University of Colorado, Denver, CO, USA

Background: Abuse of the prescription drugs gabapentin and pregabalin have increased in recent years, with severe medical outcomes and deaths 
reported. Prescribing of these agents has increased significantly, as they are often used as alternatives to opioid analgesics.

Research Hypothesis: There would be an increase in the number and severity of gabapentin and pregabalin cases that received medical toxicology consultation.

Methods: This was an 8-year multicenter analysis from a prospectively collected cohort utilizing the Toxicology Investigators Consortium (ToxIC) registry, which collects data pertaining to medical toxicology consultation. More detailed clinical information was collected starting in 2014; therefore, some data are only available for these years. Cases were included if gabapentin or pregabalin misuse or abuse was reported. Clinical and demographic data were evaluated including age, sex, coingestants, clinical manifestations, treatment, and outcome.

Results: From January 2010 to July 2017, there were 515 gabapentin and 107 pregabalin adult misuse/abuse cases reported. There were an additional 39 gabapentin and 6 pregabalin misuse/abuse cases reported in adolescents (13-18 years). During this time, the prevalence of gabapentinoid cases as a percentage of all cases logged in ToxIC's registry doubled, from $1.4 \%$ to $2.7 \%$. There were no differences in sex and most patients were middle aged (mean age 50 years gabapentin vs. 46 pregabalin). There were six gabapentin and one pregabalin-related deaths, all of which involved coingestants. The most common coingestants were sedative-hypnotics/muscle relaxants, opioids, and anti-depressants for both gabapentin and pregabalin. From 2014 to 2017, the most common clinical manifestation was central nervous system depression $(50 \%$ gabapentin, $100 \%$ pregabalin), followed by hyperreflexia/myoclonus $(30 \%, 67 \%)$ and delirium $(27 \%, 0 \%)$.

Conclusions: There was an increase in cases of gabapentin and pregabalin misuse and abuse, which parallels recent prescribing trends. Healthcare providers must be cognizant of these medications' abuse potential as we attempt to curb opioid prescribing.

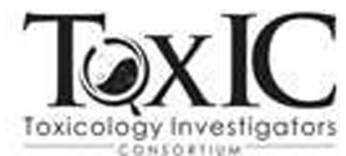

This research was performed in collaboration with the ACMT Toxicology Investigators Consortium.

072. Policy and Legislation: the Vehicles with the Means to an ENDS

Sukhshant K Atti ${ }^{1}$, Joseph Carpenter ${ }^{1}$, Alaina Steck ${ }^{1,2}$

${ }^{1}$ Emory University School of Medicine, Atlanta, Georgia, USA. ${ }^{2}$ Georgia Poison Center, Atlanta, Georgia, USA

Background: Electronic nicotine delivery systems [ENDS], including electronic cigarettes (e-cigarettes), deliver nicotine via inhaled vapor, rather than smoke, and are touted as a healthier alternative to cigarettes. From 2011 to 2015, e-cigarette use increased eightfold among high school students. This increase was attributed to branding strategies targeting youth to establish long-term clientele. This report explores recent policies and regulations surrounding e-cigarette marketing and use. Methods: We conducted a literature and legislative review regarding ecigarette policy and legislation using Pubmed, EBSCOhost, and Google Scholar. In addition, The Policy Surveillance Program, an online database and legal mapping tool, was reviewed to track relevant legislation from August 1, 2016 to August 1, 2017.

Results: In 2016, the FDA extended the Center for Tobacco Products' regulatory authority to include all tobacco products, including ENDS. From 2016 to 2017, 33 states had no laws regulating minimum age requirements surrounding use, purchase, or possession of e-cigarettes. Child-resistant packaging was required in 21 states. Three states required the package to state "keep away from children." A warning notice on the health risk of e-cigarette use was required on packaging in three states. One state, Utah, restricted the sale of e-cigarettes in and near schools, in self-service displays, and in vending machines. Only Washington State required nicotine concentration to be listed on product packaging. Thirty- six states did not have laws regarding online e-cigarette purchase. In 2018, the FDA deemed that all tobacco products, including ENDS, must include a warning of the addictive potential of nicotine. As part of its Youth Tobacco Prevention Plan, the FDA took aggressive enforcement actions targeting e-cigarette companies' misleading marketing strategies. Conclusions: Significant gaps exist in regulation of ENDS products. Policy and systems-level interventions are harm reduction strategies with potential to reduce nicotine use in the vulnerable populations of children, teens, and young adults.

\section{Kits Say the Darndest Things}

Ahmed Mamdouh Taha Mostafa, Maricel Dela Cruz, Muhammed Ershad, David Vearrier

Drexel University College of Medicine, Department of Emergency Medicine, Division of Medical Toxicology, Philadelphia, PA, USA

Background: Despite being known to trigger false positives or negatives, drug field tests (DFT) are still being used by law enforcement officers to charge suspects with possession of narcotics. No specific mention of cross-reactive substances is mentioned in the product labeling.

Hypothesis: Pharmaceuticals with similar chemical structure or properties will trigger false positives on DFT.

Methods: This is a descriptive study reporting the results of 4 different DFT kits, namely Nark II Mollies Reagent (Kit 1), NARK II MDPV Bath Salts Reagent (Kit 2), QuickCheck Marquis Tube (Kit 3), and QuickCheck Meth/Ecstasy Pouch (Kit 4) against tizanidine, selegiline, bupropion, clonidine, and lamotrigine.

Results: Tizanidine triggered a false-positive result for 2C, MDPV, or methylone by turning yellow using Kit 1, MDPV, butylone, pentylone, ethylone (bK-MDEA), methylone, or naphyrone by turning yellowishgreen using Kit 2, and methamphetamine or amphetamine by turning orange-brown using Kit 3. Selegiline triggered a false-positive result for methamphetamine or amphetamine using Kit 1 and Kit 3 by turning brown and orange respectively. The reagent in Kit 1 is clear prior to using it for testing. When it was used with lamotrigine it remained clear, which is considered a positive result. The same results were seen with bupropion, clonidine, and loratadine.

Conclusion: DFT may trigger false positives with many substances including legal pharmaceuticals. Caution should be used in interpretation of these tests.

074. Biobot: a Novel Wastewater analysis Technique to Quantify Opioid Use in Communities

Mariana Matus ${ }^{1}$, Timothy Erickson ${ }^{2,3}$, Edward Boyer ${ }^{2,4}$, Peter Chai ${ }^{2,4}$

${ }^{1}$ Biobot Inc, Boston, MA, USA. ${ }^{2}$ Division of Medical Toxicology, Department of Emergency Medicine, Brigham and Women's Hospital, Boston, MA, USA. ${ }^{3}$ Harvard Humanitarian Institute, Boston, MA, USA. ${ }^{4}$ The Fenway Institute, Boston, MA, USA

Background: Understanding community-level opioid use can help tailor public health interventions addressing this epidemic. Wastewater analysis has been successfully used to measure the consumption of illicit substances such as cocaine and methamphetamine. However, opioid metabolites have remained an open challenge in the field due to rapid degradation in the wastewater system. Biobot is a novel wastewater analysis system that provides aggregate geo-localized insights into opioid use patterns in communities. Biobot has built a platform to collect and stabilize wastewater samples from sub-city level geolocalized areas. This novel approach provides near city block level resolution and captures many metabolites that were previously inaccessible.

Hypothesis: To determine the feasibility of Biobot to collect and describe opioid use among municipalities.

Methods: We conducted a pilot study to test the feasibility of detecting metabolites of heroin, fentanyl, various prescription opioids, methadone, 
buprenorphine, and naloxone in municipal wastewater. Ten sampling stations representing communities of 4000-20,000 individuals were selected to represent a municipality. From each station, 24-h composite samples were collected with an in-house sampler, filtered and loaded into solid-phase extraction columns to concentrate metabolites. The extracts were processed and analyzed with liquid chromatography mass spectrometry. Extract concentrations were converted to collective consumption rates (mg/day/1000 people) according to published methods. Sampling took place every 2 weeks for 3 months.

Results: Through the pilot study, we showed that the Biobot sampling instrument successfully collected and stabilized 24-h composite samples, detecting all metabolites we assayed. Some of the metabolites, like naloxone, were not consistently detected in all samples indicating that sensitivity should be improved to detect substances with sporadic use.

Conclusions: Wastewater analysis is a feasible technique to measure opioid consumption in municipal communities. These data can be used to design localized public health interventions and evaluate their impact near real-time.

\section{Tianeptine Withdrawal Following Discontinuation of High-Dose Recreational Use}

Rachel Shively, Joshua Nogar

Northwell Health, Manhasset, New York, USA

Background: Tianeptine is an atypical tricyclic pharmaceutical with muopioid agonism at high doses. Used as an antidepressant abroad but lacking FDA approval in the USA, it has shown increasing prevalence as a drug of abuse. As it has both serotonergic modulation effects and muopioid agonism, its withdrawal syndrome exhibits a mixed presentation. Case Report: A 44-year-old man with history of hypertension and a 4year history of tianeptine abuse presented to the Emergency Department (ED) with concerns of tianeptine withdrawal after discontinuing this medication. He abused tianeptine for "anxiety" and an "opiate-like high," purchasing it from an online supplement store and taking $5 \mathrm{~g}$ daily for over 3 years. After becoming concerned he was addicted, he titrated himself down by $1 \mathrm{~g}$ daily over 5 days. Twenty-four hours after cessation, he had developed anxiety, palpitations, nausea, vomiting, and diarrhea. These symptoms persisted, and he presented to the ED 1 day later with vital signs: $98.7^{\circ} \mathrm{F}$, pulse 60 beats $/ \mathrm{min}$, blood pressure $128 / 74 \mathrm{mmHg}$, respiratory rate 14 breaths/min, and $100 \%$ oxygen saturation on room air. He was anxious-appearing, mildly tremulous, yawning, with hyperactive bowel sounds and $4 \mathrm{~mm}$ pupils, but without piloerection or diaphoresis. His labs and serum drug screen were unremarkable. His EKG showed sinus bradycardia: rate 56, QRS $80 \mathrm{~ms}$, and QTc $391 \mathrm{~ms}$. He received clonidine, lorazepam, gabapentin, and hydroxyzine until symptom resolution was achieved, and he had no telemetry events over a 36-hour admission.

Discussion: Tianeptine abuse has been on the rise in the USA, with $13 \%$ of calls to the National Poison Data System (NPDS) being withdrawalrelated from 2000-2017. Although high doses are abused, NPDS data shows no evidence of tricyclic antidepressant-like toxicity. Withdrawal symptoms appear to be alleviated with benzodiazepines, clonidine, hydroxyzine, gabapentin and antiemetics. Given the mechanism of action and withdrawal syndrome tianeptine causes, partial opioid agonists may be useful treatment options as well.

\section{Increasing Abuse of Propylhexedrine Reported to US Poison Centers}

Elizabeth Black ${ }^{1}$, Michael Wahl ${ }^{2}$

${ }^{1}$ Toxikon, Chicago, IL, USA. ${ }^{2}$ IL PC, Chicago, IL, USA

Background: Propylhexedrine, the active ingredient in Benzedrex®, is an OTC medication sold for relief of nasal congestion. It is structurally similar to amphetamine, with local alpha-agonist effects and systemic sympathomimetic effects. Its abuse was associated with fatalities in the 1970s, but it has been out of the public and toxicologic eye for decades. Research Question: Has there been an increase in intentional abuse/ misuse of propylhexedrine?

Methods: A retrospective review of the National Poison Data System (NPDS) for cases involving isolated, single, intentional exposure to propylhexedrine, from 2007 to 2016 was undertaken, and returned 542 cases. Clinical effects were not reported in 238 cases; these were discarded as were cases under age 13 to focus on intentional exposures, leaving 283 patients. Characteristics of these cases were analyzed using Microsoft Excel@.

Results: An increase in reports was noted from 2007 (16 cases) to 2016 (58 cases). The most common reported adverse effects were tachycardia (51.9\% of cases), agitation (33.8\%), and hypertension (28.6\%). There were also five seizures and three total dysrhythmias. No fatalities were reported.

Further, 187 (66.1\%) patients were male, 96 (33.9\%) female, with an average age of 32 years. Major clinical effects were seen in 13 patients, moderate (118), mild (101), and no effect (51).

Discussion: This study shows an increasing number of cases of intentional misuse/abuse of propylhexedrine being reported to poison centers. The majority of clinical effects are sympathomimetic, with serious sequelae of seizures and dysrhythmias captured in the data set. While the lack of reported fatalities is reassuring, the increase in cases, with a historical potential for lethal poisoning, is concerning.

Conclusion: There has been an increase in reports of propylhexedrine abuse/misuse to poison centers over the last decade. Investigation into potential causes for this increase and further monitoring for evidence of increasing use is indicated.

\section{Case Report of the LSD Analog AL-LAD Associated with Ventricular Fibrillation and Death}

Adam Blumenberg, Lauren Murphy, Tony Rianprakaisang, Adrienne Hughes, Robert Hendrickson

Oregon Health and Science University, Portland, OR, USA

Introduction: Novel psychoactive compounds are easily available to the general public via internet purchasing. AL-LAD is an LSD analogue with little information on clinical effects.

Methods: Case report of a man who developed QTc prolongation, ventricular fibrillation, and death in association with exposure to AL-LAD.

Case: A 51-year-old man with a history of hyperlipidemia and vertebral artery stenosis shot and killed two teenagers, then set their trailer on fire. Police took him into custody, described him as calm and directable, and placed him in the backseat of a police car where he was soon found pulseless and unresponsive. EMS identified ventricular fibrillation which responded to electrical defibrillation. In the ED, the patient was unresponsive with normal vitals, and physical examination showed no acute abnormalities. An electrocardiogram showed sinus rhythm with a corrected QT interval (QTc) of $536 \mathrm{~ms}$. CT of the head and chest radiograph were normal, and a formal echocardiogram showed an ejection fraction between $45-50 \%$. Laboratory analysis was notable for normal potassium and magnesium, and mild hypocalcemia $(7.9 \mathrm{mg} / \mathrm{dL})$ when corrected for albumin. The QTc decreased to $476 \mathrm{~ms}$ the next day. He developed anoxic brain injury, the family withdrew care, and he died a week later. Urine drug immunoassay and high performance liquid chromatography/time of flight mass spectrometry (LC/TOF-MS) were used to identify AL-LAD exposure and to eliminate other toxicants which may have contributed to the lethal dysrhythmia. Discussion: This case adds to the medical literature a description of toxicity from AL-LAD with serologically confirmed AL-LAD exposure. AL-LAD appears to have led to delusions and erratic behavior, which are expected effects of LSD analogues. Unique aspects of the case include the prolongation of the QTc and ventricular dysrhythmia.

Conclusion: Physicians should be aware of the potential for sudden cardiac death associated with AL-LAD exposure. 
078. Case of Somnolence Due to Ingestion of a Liquid Designer Benzodiazepine

Lauren Murphy, Adam Blumenberg, Adrienne Hughes, Tony Rianprakaisang, Robert Hendrickson

Oregon Health and Science University, Portland, OR, USA

Background: Clonazolam is a designer triazolobenzodiazepine first synthesized in the 1970s that has never been marketed for medicinal use. Currently not classified as a controlled substance, clonazolam first surfaced on the internet in 2014 as a drug of abuse.

Methods: This is a single patient case review. A 28-year-old male patient presented with somnolence to an emergency department after reportedly ingesting one-half of a $30 \mathrm{ml}$ vial of liquid clonazolam he purchased from the internet for recreational purposes. The label displayed a concentration of $0.5 \mathrm{mg} / \mathrm{ml}$, a total volume of $30 \mathrm{ml}$, and contents listed as clonazolam, ethyl alcohol, and propylene glycol. The liquid was colorless and clear. He had normal vital signs and had no distress on presentation. We confiscated the vial for safety and for QTOF-MS testing. He underwent unremarkable screening laboratory tests and his mental status cleared during a six-hour observation in the emergency department.

Results: Clonazolam was identified by QTOF-MS from the contents of the vial, without the presence of other pharmaceuticals.

Discussion: Increasing numbers of cases of intoxication involving clonazolam have been identified since 2016 in Europe, most notably in Sweden and Norway, with a single case reported in the USA in 2017. It is typically found in tablet, capsule, pellet, blotter, or powder form. This case is unique in that it is the second case of intentional clonazolam ingestion reported in the USA, and additionally that the substance was found in liquid form.

Conclusion: Providers should be aware that clonazolam is available on the internet in liquid formulation, which may increase the risk of accidental overdose.

079. Ketamine-Induced Cholangiopathy and Uropathy: a Case Report

Mitchell Bellis, Lauren Cooke, Joshua Nogar

Northshore University Hospital, Manhasset, NY, USA

Background: Ketamine is an NMDA receptor antagonist similar in structure to phencyclidine (PCP) and is used as a drug of abuse due to its hallucinatory and dissociative effects. Chronic ketamine abuse has long been associated with uropathy, but its hepatobiliary effects are rare. We present a case of ketamine-induced cholangiopathy (KIC) and concomitant ketamine-induce uropathy (KIU).

Case: A 35-year-old man presented to the Emergency Department (ED) complaining of chronic abdominal pain. His medical history was significant for chronic daily ketamine abuse and culture-negative cystitis. $\mathrm{He}$ endorsed weeks of vomiting and constant abdominal pain despite selfmedicating with oxycodone. His vital signs were HR 106 beats/min, BP $151 / 110 \mathrm{mmHg}$, RR 19 breaths $/ \mathrm{min}$, Temp $98.8^{\circ} \mathrm{F}$, and $\mathrm{O}_{2}$ saturation $99 \%$ on room air. Physical exam was significant for moderate distress and a diffusely tender abdomen. Laboratory studies were significant for an alkaline phosphatase of $123 \mathrm{IU} / \mathrm{L}$, and the urinalysis showed microscopic hematuria. His AST/ALT and bilirubin were normal, as was his $\mathrm{CBC}$, lipase, and the remainder of his metabolic panel. A CT abdomen demonstrated intrahepatic dilation of the common bile duct (CBD) and bladder wall thickening consistent with cystitis. An abdominal ultrasound confirmed a $16 \mathrm{~mm}$ CBD and an enlarged gallbladder without signs of cholecystitis, stones, or sludge. During a similar presentation 8 months prior, his CBD was $8 \mathrm{~mm}$ on abdominal US, and neither his MRCP nor HIDA scans demonstrated choledocholithiasis, obstruction, stricture, or cholecystitis. The patient was ultimately diagnosed with KIC and KIU.

Discussion: Although the mechanism of injury is unclear, ketamine is a rare but recognized cause of cholangiopathy in heavy ketamine abusers.
Case reports from Asia demonstrate laboratory and biopsy findings of cholestatic liver injury that improve after cessation of ketamine abuse. Providers should be aware of ketamine's potential to cause cholestatic liver injury.

\section{Phenibut Exposures Reported to New Jersey Poison Control Center}

Edmund Rurup ${ }^{1}$, Bruce Ruck ${ }^{2}$, Lewis Nelson ${ }^{3,1}$, Diane Calello ${ }^{1,3}$

${ }^{1}$ New Jersey Poison Information and Education System, Newark, NJ, USA. ${ }^{2}$ New Jersey Poison Information and Education System, Newark, NJ. ${ }^{3}$ Department of Emergency Medicine, Rutgers New Jersey Medical School, Newark, NJ, USA

Background: Phenibut (4-amino-3-phenyl-butyric acid), a GABA receptor agonist with anxiolytic and stimulant properties, is sold legally outside the USA. It is touted on internet addiction and drug use forums for its putative effects, which include enhanced cognitive performance (nootropism), increased sociability, euphoria, and anxiolysis.

Research Question: What are the features of phenibut exposure reported to one poison center?

Method: Review of phenibut exposures reported to the NJ Poison Center between 2014 and 2018. Exposures were reviewed for demographics, circumstances, clinical effects, duration of effects, and AAPCC medical outcomes.

Results: Twenty cases of phenibut exposure were reported from 2014 to 2018; the incidence more than doubled in 2018. Demographics: $90 \%$ male, age 21 months-68 years (median 29 years). Circumstances: $50 \%$ $(n=10)$ intentional abuse or misuse, $15 \%(n=3)$ intentional suspected suicide, $15 \%(n=3)$ intentional with unknown motivation, $10 \%(n=2)$ unintentional, and $10 \%(n=2)$ suspected withdrawal from the substance. The most common effects were drowsiness $(40 \%, n=8)$ and confusion $(30 \%, n=6)$. Duration of symptoms were less than $2 \mathrm{~h}(5 \%, n=1)$, between 2 and $8 \mathrm{~h}(25 \%, n=5)$, between 2 and $8 \mathrm{~h}, 8-24 \mathrm{~h}(25 \%, n=$ $5), 24 \mathrm{~h}-3$ days $(10 \%, n=2)$, and up to a week in $15 \%(n=3)$. Medical outcomes were $10 \%(n=2)$ no effect, $20 \%(n=4)$ minor effect, $50 \%(n=$ $10)$ moderate effect, $10 \%(n=2)$ major effect, and $10 \%(n=2)$ unrelated effect.

Discussion: During the study period, we observed an increase in phenibut exposures. Most were misuse/abuse, had moderate, minor, or no effects, and resolved within $24 \mathrm{~h}$. Although the at-risk population, full range of clinical effects, and risks are not yet clear, this raises concerns for increased unregulated use in the community.

Conclusions: Phenibut is an emerging substance of misuse. Although exposures are relatively uncommon, clinicians should be alert to the potential for this exposure, particularly in patients with drug abuse and misuse.

081. A Comparative Analysis of Kratom Abuse in Thailand and the United States from 2010 to 2017

Christian Davidson ${ }^{1}$, Taylore King ${ }^{1}$, Sunun Wongvisavakorn ${ }^{2}$, Natthasiri Ratprasert ${ }^{2}$, Satariya Trakulsrichai ${ }^{2}$, Dazhe $\mathrm{Cao}^{1}$, Sahaphume Srisuma $^{2}$ ${ }^{1}$ University of Texas Southwestern Medical Center, Dallas, Texas, USA. ${ }^{2}$ Ramathibodi Hospital, Bangkok, Thailand

Objective/Methods: This retrospective analysis describes and compares characteristics, co-abuse substances, clinical effects, treatments, and outcomes of kratom abuse exposures reported to the Ramathibodi Poison Center (RPC) in Thailand and the National Poison Data System (NPDS) in the United States (U.S.) from 2010 to 2017. Subgroup analysis of both single- and multiple-substance exposures was performed. NPDS was used as reference for comparative analysis. Severe outcomes were defined as major clinical effects or fatal outcomes.

Results: Nine hundred twenty-eight total cases were included (760 from NPDS, 168 from RPC). There was a higher proportion of kratom co- 
abuse in Thailand $(65.0 \%$ and $35.0 \%$ in Thailand and USA respectively; odds ratio [OR] 3.10, 95\% confidence interval [95\% CI] 2.15-4.47). Ethanol, opioids, and stimulants were the most commonly co-abused substances in both countries. There were more reports of stimulant coabuse in Thailand $(21.43 \%$ and $2.89 \%$ in Thailand and USA respectively; OR 9.15, [95\%CI] 5.04-16.82) but no difference in ethanol or opioid coabuse rates between countries. Common clinical effects were tachycardia (30.4\%), agitation/irritability (26.2\%), and drowsiness/lethargy $(21.1 \%)$. There were more reports of dystonia in Thailand (OR 15.89, [95\%CI] $5.43-56.07$ ) and more coma in the USA (OR 0.23, [95\% CI] 0.05-0.72). Common treatments in both countries were IV fluids (40.1\%), benzodiazepines $(27.3 \%)$, and oxygen (10.9\%). There were six deaths, including one single-substance exposure and three multiple-substance exposures in the USA, and two multiple-substance exposures in Thailand. Singlesubstance kratom abuse resulted in 48 cases $(10.1 \%)$ with severe outcomes in the USA, whereas none occurred in Thailand $(0)(\mathrm{OR} 0$, [95\%CI] 0-0.58). In multiple-substance abusers, 58 cases $(20.4 \%)$ in the USA and 3 cases (2.8\%) in Thailand reported severe outcomes (OR $0.11,[95 \% \mathrm{CI}]$ 0.02-0.35).

Conclusions: Kratom abuse in Thailand is more likely to involve multiple-substance exposure than in the U.S.; however, severe medical outcomes were reported more frequently in the USA in both single- and multiple-substance kratom abuse.

082. Pneumomediastinum, Acute Kidney Injury, Rhabdomyolysis, and Cryogenic Dermal Injuries Secondary to Inhalation Abuse of Keyboard Cleaner

Mayur Patel, Mohan Punja, Richard Kleiman, Menelaos Demestihas WellStar Kennestone Regional Medical Center, Marietta, Georgia, USA

Background: Inhalant abuse is the inhalation of volatile substances, such as halogenated hydrocarbons found in keyboard cleaner. Cryogenic injuries are well-known complications of keyboard cleaner abuse; pneumomediastinum, renal injury, and rhabdomyolysis are less common. Hypothesis: Inhalation abuse of halogenated hydrocarbons can lead to renal injury, rhabdomyolysis, barotrauma and pneumomediastinum, and cryogenic dermal injuries.

Methods: This is a single patient chart review.

Case: A 31-year-old previously healthy male presented to the Emergency Department by ambulance after inhaling ten cans of "Dust Off" keyboard cleaner and was found unconscious in his car. Vital signs revealed mild tachycardia and tachypnea. He was awake, alert, and not in acute distress. Multiple cryogenic burns were noted along his neck and chest. Computed tomography scan showed subcutaneous air in the base of the neck and mediastinum. Serum studies were notable for creatinine $(\mathrm{Cr})$ of $2.9 \mathrm{mg} / \mathrm{dL}$ and creatine kinase (CK) of $1188 \mathrm{IU} / \mathrm{L}$. The final diagnosis was acute kidney injury and pneumomediastinum secondary to inhalant abuse of 1,1 difluoroethane with multiple cryogenic burns and rhabdomyolysis.

Discussion: The product abused by this patient contains 1,1 difluoroethane, a fluorinated hydrocarbon that causes CNS depression, respiratory irritation, arrhythmias, and cryogenic mucosal, and dermal injuries. The compound is largely exhaled unchanged with renally excreted metabolites. Exposure to the rapidly cooled container resulted in cryogenic burns; elevated CK suggests mild rhabdomyolysis. He developed pneumomediastinum secondary to barotrauma of the tracheobronchial tree and esophagus. The increased $\mathrm{Cr}$ may be due to direct kidney injury from metabolites of 1,1 difluoroethane and/or renal filtration of myoglobin.

Conclusion: The patient was admitted to the hospital service for management of his pneumomediastinum and renal injury. Cardiothoracic surgery recommended conservative management and broad spectrum antibiotics/antifungals. His renal injury was managed with intravenous hydration. The patient left against medical advice after three days of inpatient treatment.
083. Brief Asystole in a Four-Year-Old Following Ingestion of Cannabis Edibles

Laurie Halmo ${ }^{1}$, Caitlin Bonney ${ }^{1}$, Benjamin Hatten ${ }^{2}$

${ }^{1}$ Rocky Mountain Poison and Drug Center, Denver, CO, USA.

${ }^{2}$ University of Colorado School of Medicine, Aurora, CO, USA

Background: Previous reports have described cannabis exposure associated with the development of serious cardiac events including myocardial infarction, cardiomyopathy, dysrhythmias, and cardiac arrest. As access to cannabis becomes more widespread in the USA, reports of cannabis-related cardiac dysfunction in pediatric patients are emerging. Methods: This is a case report of a 4-year-old previously healthy female who was found somnolent and ashen at home. The patient's mother suspected ingestion of $20 \mathrm{mg}$ of cannabis in the form of edible gummies. The patient was transported to an emergency department for evaluation. Results: The patient's initial vital signs were remarkable only for a heart rate of 119 beats $/ \mathrm{min}$. and a blood pressure of $114 / 71 \mathrm{mmHg}$. While on telemetry monitoring, she experienced an eight-second episode of asystole with concurrent respiratory arrest. The child regained pulse and respirations without intervention; immediately post-arrest her heart rate was 44 beats $/ \mathrm{min}$ and her systolic blood pressure was $60 \mathrm{mmHg}$. After an infusion of $20 \mathrm{~mL} / \mathrm{kg}$ normal saline, her vital signs normalized. A urine drug screen was positive only for cannabinoids. The remainder of her laboratory analyses, including a complete blood count, comprehensive metabolic panel, venous blood gas, acetaminophen concentration, salicylate concentration, and ethanol concentration were unremarkable, as was a noncontrast computed tomography scan of her head. She was transferred to a pediatric intensive care unit where her vital signs remained normal and was discharged following pediatric electrophysiology consultation.

Conclusion: Case reports have documented symptomatic bradycardia and asystole in adults with a history of repeated cannabis use. We report a case of a previously cannabis-naïve young child who developed asystole after exposure to marijuana edibles. Cannabis-related cardiotoxicity in children warrants further study.

084. Prevalence of Marijuana use Among Trauma Patients Before and After Medical Marijuana Became Legal

Michael Levine ${ }^{1}$, Ashley Jontz ${ }^{2}$, Paul Dabrowski ${ }^{2}$, Noa Yee ${ }^{3}$, Ilene Claudius $^{4,5}$, Rachel Kreisler ${ }^{6}$, Frank LoVecchio ${ }^{2}$

${ }^{1}$ University of Southern California, Los Angeles, CA. ${ }^{2}$ Banner University Medical Center, Phoenix, Phoenix, AZ. ${ }^{3}$ University of Hawaii School of Medicine, Honolulu, HI. ${ }^{4}$ University of California, Los Angeles, Los Angeles, CA. ${ }^{5}$ Harbor UCLA Medical Center, Torrance, CA. ${ }^{6}$ Midwestern University, Glendale, AZ

Background: Medical marijuana cards became available in Arizona (AZ) on 4/14/2011; patients with marijuana cards could grow cannabis, although dispensaries did not open until 12/6/2012. The degree to which legalization and availability of marijuana impacts trauma patients remains largely unstudied.

Hypothesis: We suspected an increase in prevalence post-legalization. Methods: This study evaluates the prevalence of marijuana use among trauma patients in three periods: $1=$ before legalization of medical marijuana $(1 / 1 / 2011-4 / 13 / 2011) ; 2$ = legal to use, but no dispensaries $(4 / 14 /$ 2011-12/5/2012); 3 = legal to use and purchase at dispensaries (12/6/ 2012-9/30/2014) in Phoenix, AZ. Marijuana use was defined either by self-reporting or positive urine drug screen (UDS), which was routinely obtained on trauma patients. The prevalence of marijuana use in patients presenting pre-legalization and post-legalization was compared using multivariate logistic regression.

Results: A total of 5373 subjects were identified. Further, 115 subjects were excluded (110 unable to respond regarding drug use/ no UDS; 5 age $<13)$. The prevalence of marijuana per time period was: time 1: $18.1 \%$ 
(95\% CI 15.3-21.1), time 2: $22.4 \%$ (95\% CI 20.8-24.1), time 3: $20.1 \%$ (95\% C 18.5-21.9). The odds ratio of being marijuana positive postlegalization vs. pre-legalization (periods 2 and 3 vs. 1) was 1.38 (95\% CI $1.11-1.72, p=0.004)$. There was no difference between periods 2 and 3 . As compared to time 1 , the odds ratio of being marijuana positive postlegalization for males was 1.49 (95\% CI $1.17-1.89, p=0.001)$. Females were less likely than males to be positive pre-legalization (OR 0.37).

Conclusion: Legalization of medical marijuana is associated with increased use among male trauma patients. The legalization rather than the convenience of dispensaries appears to be associated with this increase.

085. Acute Cannabis Exposures in Children Cause Sedation more Often than Excitation, Whereas the Opposite Is True for Adults

Matthew Noble, Robert Hendrickson

Oregon Health and Science University, Portland, OR, USA

Background: Tetrahydrocannabinol(THC) is legal in 31 states as "medicinal cannabis" and in 9 states as "retail cannabis." Previous studies have noted a high rate of central nervous system (CNS) depression in pediatric subjects, although most of these data have been limited to exposures to edible cannabis products.

Research question: Do children suffer CNS sedation more commonly after acute exposure to cannabis products than adults?

Methods: Prospective study of subjects where the Oregon Poison Center (OPC) was contacted about a cannabis exposure between 12/4/2015 and 4/15/2017. A data collection sheet prospectively captured clinical and product data, including method of use and cannabis type. PC charts were searched for cannabis substance codes. Inclusion criteria: acute cannabis exposure to a single known product. Exclusion criteria: co-ingestion of other medications/drugs. Chi square and Fisher's exact test were used to compare frequencies of events.

Results: In children ( $<12$ years), CNS sedation without excitation occurred more often overall and for each product category, compared to CNS excitation without sedation: $28(43.1 \%)$ vs $6(9.2 \%)$ of 65 total exposures, including $22(46.8 \%)$ vs. $5(10.6 \%)$ of 47 edible exposures, 4 $(36.4 \%)$ vs. $1(9.1 \%)$ of 11 botanical exposures, and $2(28.6 \%)$ vs. 0 $(0 \%)$ of 7 concentrated product exposures. In adults ( $\geq 18$ years), CNS sedation without excitation, compared to CNS excitation without sedation, occurred less frequently overall and in each product category: $22(15.9 \%)$ vs. $56(40.6 \%)$ of 138 total exposures, including $11(16.2 \%)$ vs. $24(35.3 \%)$ of 68 edible exposures, $2(8.0 \%)$ vs. 12 (48.0\%) of 25 botanical exposures, and $9(20.0 \%)$ vs. $20(44.4 \%)$ of 45 concentrated product exposures.

Conclusion: Although there were inherent limitations in exposures reported to OPC, children were more likely to experience CNS sedation after exposure to cannabis, whereas adults were more likely to experience CNS excitation $(p<0.01)$

086. A Case Series of Heroin Adulterated with the Novel Synthetic Cannabinoid, 5F-MDMB-PINACA, in Philadelphia, Pennsylvania, USA

Maricel Dela Cruz, Muhammed Ershad, Ahmed Mamdouh Taha Mostafa, Muhammad Khalid, Ryan Arnold, Richard Hamilton Drexel University College of Medicine, Philadelphia, PA, USA

Background: Heroin is often adulterated with a variety of substances including baking soda, caffeine, crushed analgesics, and scopolamine. We present a series of patients with similar presentations after overdose with intravenous (IV) heroin adulterated with the synthetic cannabinoid (SC) 5F-ADB (5F-MDMB-PINACA).

Hypothesis: Heroin contaminated with the SC, 5F-ADB exhibits anticholinergic effects that are successfully treated with physostigmine.

Methods: This is a consecutive-patient case series. Within 3 months, eight patients arrived to the emergency department after use of IV heroin with symptoms consistent with anticholinergic toxicity and variable requirement for naloxone administration by emergency medical services. Cases 1-4 used IV heroin-labeled "Santa Muerte," and cases 5-8 used IV heroin from a bag similar to "Santa Muerte," but labeled "50Cal" or "Nick."

Results: Gas chromatography mass spectrometry (GCMS) of the drug samples exhibited heroin, fentanyl, and 5F-ADB. Cases 1-4 presented with an anticholinergic toxidrome after opioid antagonism and were successfully treated with physostigmine. Case 1 and 3 developed ARDS requiring VV-ECMO. Case 3 was transferred to a tertiary care center. Case 4 required benzodiazepine treatment in addition to physostigmine to reverse symptoms. Cases 5-8 presented after confirmed IV heroin use with an agitated delirium consistent with a predominant anticholinergic toxicity and were treated with physostigmine and benzodiazepines successfully without the requirement for opioid antagonism, and only minor symptoms of opioid toxicity.

Discussion: Heroin contaminated with 5F-ADB exhibits variable severities of anticholinergic effects, some on presentation and others only after opiate antagonism. SCs affect cannabinoid CB1 and CB2 receptors, potentially causing adrenergic stimulation, sedation, hallucination, catecholamine release, and severe tachycardia.

Conclusion: Heroin contaminated with the SC 5F-ADB exhibited varied symptoms, including anticholinergic toxicity.

\section{Novel Ketamine Analogs Cause a False-Positive Phencyclidine} Immunoassay

John Skaugen, Anthony Scoccimarro, Anthony Pizon, Jacqueline Rymer, Spiros Giannoutsos, Sean Ekins, Matthew Krasowski, Kenichi Tamama University of Pittsburgh Medical School, Pittsburgh, PA, USA

Background: Immunoassays are commonly used as a screening tool in patients presenting with xenobiotic poisoning. Ketamine derivatives, such as deschloro- $N$-ethyl-ketamine (eticyclidone or 2-oxo-pCE), share structural homology with phencyclidine (PCP), and have become more prominent as drugs of abuse.

Hypothesis: 2-oxo-PCE causes a false positive PCP screen.

Methods: This is a single patient case report. A 27-year-old man was brought to the emergency department after being found with a change in mental status. He stated he had purchased 2-oxo-PCE through the "dark web" and had been using it for the 4 days prior to presentation. Physical exam was notable for tachycardia, bilateral nystagmus, and multiple abrasions throughout his extremities and torso. Laboratory testing was notable for acute kidney injury and mild rhabdomyolysis. He was treated with supportive care for 2 days and returned to baseline neurologic and renal function.

Results: Urine enzyme multiplied immunoassay technique (EMIT) on the Siemens Viva-E platform (Siemens Healthcare Diagnostics, Inc., Tarrytown, NY, USA) was positive for benzodiazepines and PCP. Urine gas chromatography/mass spectrometry (GC/MS) was positive for 2-oxo-PCE (large peak), ketamine, salicylate metabolite, naproxen, and caffeine. We conducted urine spiking studies and $2 \mathrm{D}$ chemical similarity calculations for ketamine and several analogs to assess their structural similarity to PCP and crossreactivity on the EMIT II PCP immunoassay. These analogs, including 2-oxo-PCE and others [3-methoxyphencyclidine (3-MeOPCP), 3-methoxyeticyclidine (3-MeO-PCE), and methoxetamine (MXE)], cause false-positive PCP immunoassay results, while ketamine gives a negative result.

Discussion: The cross-reactivity data agree with 2D molecular similarity calculations for these molecules, validating the robustness of $2 \mathrm{D}$ molecular similarity analysis in predicting molecular cross-reactivity. Ketamine analogs cause a false-positive PCP immunoassay, although ketamine does not. These xenobiotics appear to have similar clinical effects.

Conclusions: Due to structural homology, novel ketamine analogs such as 2-oxo-PCE cause a false-positive PCP immunoassay. 
088. Prolonged Psychosis After 4-bromo-2,5-Dimethoxyamphetamine use Confirmed by GC-MS

Takuyo Chiba ${ }^{1,2}$, Katherine Boyle ${ }^{1,2}$, Michael Ganetsky ${ }^{1,2}$

${ }^{1}$ Harvard Medical Toxicology Program, Boston Children's Hospital Boston, Boston, MA, USA. ${ }^{2}$ Division of Medical Toxicology, Department of Emergency Medicine, Beth Israel Deaconess Medical Center, Harvard Medical School, Boston, MA, Boston, MA, USA

Background: 4-Bromo-2,5-dimethoxyamphetamine (DOB) is a methoxyamphetamine derivative with similar effects to lysergic acid diethylamide (LSD). There are several confirmed reports in the literature, mostly occurring in the 1980 s.

Hypothesis: Prolonged psychosis is associated with DOB use.

Methods: This is a single case report.

Results: A 23-year-old female without any prior medical history or family history of psychiatric illness presented with acute agitation and delusion after ingesting "LSD" the day before. She believed that "a bomb went off," which had killed or was about to kill her. She later reported use of LSD 3-4 times in the past, and occasional marijuana use. After $14 \mathrm{mg}$ of IV lorazepam, dexmedetomidine infusion was started for treatment of refractory agitation, and continued intermittently for $46 \mathrm{~h}$. Haloperidol was also initiated per psychiatry recommendation. She was discharged on day 6 with a normal mental status, but she represented $12 \mathrm{~h}$ later with the agitation and psychosis, which continued until day 10 . She and her family, who closely monitored her, denied re-exposure. Urine from day 6 was sent to NMS Labs for analytical testing of LSD, NBOMe derivatives, novel psychoactive substances, hallucinogens, and stimulants; qualitative gas chromatography mass spectrometry was positive for DOB. Serum alcohol concentration, and urine drug of abuse immunoassay, including amphetamine and cocaine, were negative on both initial and second presentations.

Discussion: We report confirmed DOB use associated with prolonged psychosis for 10 days. Other possible contributing factors were marijuana use and any undiagnosed psychiatric illness. Despite rare confirmed reports of DOB, this case suggests ongoing presence of $\mathrm{DOB}$ in the recreational hallucinogen supply and should be considered when patients have prolonged psychosis.

Conclusion: Psychosis lasting for several days is not uncommon after designer hallucinogen use. We present a confirmed DOB case, with rare prior reports in the literature.

\section{Getting High at What Cost? Case Series of 3-MeO-PCP} Intoxication in a Single Patient

Alexander Sidlak, Joshua Shulman, Ryan Marino, Anthony Scoccimarro, Shirley Shao

University of Pittsburgh School of Medicine, Pittsburgh, PA, USA

Background: 3-Methoxy-phencyclidine (3-Meo-PCP) is a novel arylcyclohexamine analogue, which was first described in the 1970s. Reports of recreational use only arose in 2011. Arylcyclohexamines were originally designed for anesthetic use but are often used recreationally. 3Meo-PCP has three times greater affinity for the NMDA receptor than PCP, but reports on its clinical effects are limited.

Methods: A 24-year-old transgender woman presented ten times over 7 months to the emergency department. On four occasions, she had a urine drug screen positive for PCP later identified by gas chromatography/mass spectrometry (GC/MS) to have been triggered by the use of 3-MeO-PCP. She admitted to using "PCP," reporting use of between 25 and $50 \mathrm{mg}$ by mouth daily.

Results: In three out of the four visits, the patient presented comatose requiring intubation for respiratory failure once. Tachycardia (113$115 \mathrm{bpm})$ and systolic hypertension $(144-160 \mathrm{mmHg}$ ) were seen on three visits. The patient developed mild rhabdomyolysis (CK, $5356 \mathrm{IU} / \mathrm{L}$ ) on one presentation where 3-MeO-PCP was the sole ingestion. She improved over the course of $24 \mathrm{~h}$ on all occasions. Absence or presence of nystagmus was not always documented. Co-ingestions present on three occasions included $\mathrm{N}$-ethyl-4-fluoroamphetamine, tramadol, and ethanol. Discussion: A case series of isolated 3-Meo-PCP intoxications from 2015 reported tachycardia, hypertension, nystagmus, and altered mental status as the most common symptoms, which is consistent with this patient's presentations. The one episode of moderate coma with respiratory failure requiring intubation appeared to be an isolated overdose given a negative ethanol level and only detection of the patient's home medications on urine GC/MS.

Conclusion: 3-MeO-PCP is a recreational illicit drug rarely encountered in the USA. We present a case series of 3-MeO-PCP ingestions in a single patient associated with clinically significant toxicity illustrating the potential for severe effects.

\section{Smoking the Headlines: Recurrent Carboxyhemoglobinemia with Polycythemia}

Alexander Sidlak, Ryan Marino, Anthony Pizon, Anthony Scoccimarro, Shirley Shao, Michael Lynch

University of Pittsburgh School of Medicine, Pittsburgh, PA, USA

Background: Carbon monoxide (CO) is formed by incomplete combustion of carbon containing compounds. Cigarette use can cause mild elevations in baseline carboxyhemoglobin levels (COHgb, 6-15\%), but smoking alone is not reported to cause significant elevations in the absence of other exposures. Hypothesis: Smoking hand-rolled newspaper "cigars" causes severe carboxyhemoglobinemia.

Methods: This is a single patient case report. A 58-year-old man with history of COPD, new-onset secondary polycythemia, and schizophrenia was sent to the emergency department with a $\mathrm{COHgb}$ of $35 \%$. He was a chronic smoker and rolled his own "cigars" made primarily from newspaper. On arrival, his COHgb was $38.6 \%$. He had no systemic symptoms, metabolic derangements, or focal neurologic deficits. He did not tolerate an attempt at hyperbaric oxygen therapy, and he was continued on supplemental oxygen with improvement in his $\mathrm{COHgb}$ to $3.6 \%$. Two weeks later, the patient returned with $\mathrm{COHgb}$ of $34 \%$; he was re-admitted on oxygen and improved within $24 \mathrm{~h}$. Three months later, the patient had repeat testing showing a $\mathrm{COHgb}$ of $28.8 \%$.

Results: Upon investigation, no home or occupational sources of exposure were identified. We considered methylene chloride ingestion; however, formic acid and dichloromethane levels were undetectable. His hemoglobin had been elevated (17.3-18.8 g/dL) for 8 months. A hemoglobinopathy screen showed no fetal hemoglobin. Testing for JAK2 V617F mutation was negative and an erythropoietin level was $23.5 \mathrm{mIU} / \mathrm{mL}$, confirming secondary polycythemia.

Discussion: This patient's frequent smoking of hand-rolled newspaper "cigars" was the likely etiology of his symptoms. On cessation of smoking his levels would improve, but they recurred when he resumed smoking as an outpatient. Other causes were excluded through laboratory analysis and home evaluation.

Conclusion: Due to the patient's compulsion for smoking newspaper "cigars," he developed recurrent severe carboxyhemoglobinemia associated with polycythemia, but no chronic systemic symptoms.

091. Carbon Monoxide Toxicity in a Tobacco Smoker After Switching from Cigarettes to Cheaper Unfiltered Cigars

Alyrene Dorey, Pieter Scheerlinck, Timothy Albertson University of California Davis, Sacramento, CA, USA

Background: Carbon monoxide (CO) is a known complication of cigarette smoking and carboxyhemoglobin $(\mathrm{COHb})$ levels are generally less than $10-15 \%$ in heavy smokers. Smoke from cigars is generally not inhaled. Two cigar smokers who chronically inhaled smoke after switching from cigarettes to cigars were found to have polycythemia and elevated $\mathrm{COHb}$ 
(13\% and $15 \%)$. We report a former cigarette smoker found to have markedly elevated $\mathrm{COHb}$ after switching to inhaled cigar smoking.

Case: A 65-year-old man with chronic obstructive pulmonary disease was found to have hemoglobin $19.2 \mathrm{~g} / \mathrm{dl}$ compared to a baseline 1 year earlier of $15.1 \mathrm{~g} / \mathrm{dl}$. A COHb level ordered for evaluation of polycythemia returned at $19.4 \%$. The patient was asymptomatic. The local energy company inspected his home and found normal ambient $\mathrm{CO}$ levels. There were functional $\mathrm{CO}$ detectors in his workplace. A repeat $\mathrm{COHb} 1$ month later was $18.5 \%$ (without hemolysis) and he was referred to our clinic. His vital signs and physical exam were normal. He had no symptoms of $\mathrm{CO}$ toxicity and no environmental or occupational exposures to CO. He reported a 40-year pack-per-day smoking history. On further questioning, he reported that 1 year prior, he had switched from smoking filtered cigarettes to cigars due to the increasing cost of filtered cigarettes. The patient did not smoke for approximately $24 \mathrm{~h}$ prior to his next laboratory evaluation, which detected a $\mathrm{COHb}$ level of $7.6 \%$

Discussion: Increasing cigarette prices may cause smokers to switch to cheaper tobacco cigars. These individuals could be at increased risk of adverse health effects resulting from chronic $\mathrm{CO}$ toxicity and inhalation of unfiltered tobacco smoke. Similar risks might be extrapolated to individuals who smoke "blunts"-hollowed out cigars filled with marijuana - a common practice among some sub-populations of marijuana users. These groups may benefit from targeted preventative education.

\section{QTe Interval Prolongation Associated with Inpatient Azithromycin Therapy for Pneumonia}

Maricel Dela Cruz, Ahmed Mamdouh Taha Mostafa, Muhammed Ershad, Matthew Abrishamian, David Vearrier

Drexel University College of Medicine, Philadelphia, PA, USA

Background: In 2013, the United States Food and Drug Administration issued a warning regarding the use of azithromycin and risk of fatal dysrhythmias after a retrospective analysis in which patients who had ever received a course of azithromycin experienced an increased risk of cardiovascular related death compared to non-azithromycin users.

Hypothesis: Azithromycin has been associated with QTc prolongation in healthy controls; we seek to quantify QTc prolongation associated with azithromycin in hospitalized patients with pneumonia.

Methods: This is a retrospective study comparing QTc interval measurements on ECG before and after inpatient azithromycin treatment for pneumonia. We reviewed 346 charts from January 1 to June 30, 2017. A total of 61 patients had a pre- and post-ECG performed after one dose of azithromycin (group 1) and 22 patients had a pre- and post-ECG performed after two doses of azithromycin (group 2). QTc measurements were calculated manually utilizing Fridericia's formula in lead II. SPSS statistical software was used to assess for statistical significance.

Results: QTc interval was significantly longer after one dose of azithromycin ( $442 \mathrm{~ms}$ vs. $420 \mathrm{~ms}, p=0.005)$. QTc interval after two doses was significantly greater than pre-azithromycin ( $445 \mathrm{~ms}$ vs. $417 \mathrm{~ms}, p=$ 0.018 ), but not significantly greater than after one dose. A total of five patients in group 1 and three patients in group 2 had a QTc interval greater than $500 \mathrm{~ms}$. The average age of patients in groups 1 and 2 were 58 and 61 years old, respectively.

Discussion: Although QTc interval increased after inpatient azithromycin therapy for pneumonia, few patients had a QTc greater than $500 \mathrm{~ms}$. No patients experienced dysrhythmias during hospitalization.

Conclusion: QTc interval increase occurred during inpatient azithromycin therapy for pneumonia but was not found to be associated with any clinically significant outcomes.

093. Don't Clean Your Inhaler with Jewelry Cleaner: a Case of Unintentional Cyanide Poisoning in a Hmong-American Man

Sneha Gullapalli ${ }^{1}$, Kathryn Bilello ${ }^{1}$, Patil Armenian ${ }^{2}$

${ }^{1}$ UCSF Fresno, Div. of Pulmonary and Critical Care Medicine, Fresno, CA, USA. ${ }^{2}$ UCSF Fresno, Dept. of Emergency Medicine, Fresno, CA, USA
Background: Hmong-Americans are an ethnic group of Southeast-Asian descent inhabiting Central California, Minnesota, and Wisconsin. Using traditional household products from ethnic markets is common practice in these communities, including jewelry cleaners that contain sodium cyanide.

Methods: This is a case report.

Results: A 39-year-old Hmong-American male with asthma presented to the emergency department (ED) with 2 days of dyspnea. Just prior to symptom onset, he had cleaned his home and metered dose inhaler with a "cleaning solution" purchased at a Hmong-American store. At ED presentation, his temperature was $100.6^{\circ} \mathrm{F}$, heart rate was $110-130$ beats/min, respiratory rate was $20-24$ breaths/min, and blood pressure was $150 / 100 \mathrm{mmHg}$. He appeared flushed, with normal breath sounds and mental status. Serum laboratory tests revealed an elevated lactate [10 mmol/L] with anion gap metabolic acidosis [bicarbonate $16 \mathrm{mmol} / \mathrm{L}$, anion gap $17 \mathrm{mmol} / \mathrm{L}]$. Toxicology was consulted who suspected cyanide poisoning and recommended hydroxocobalamin $[5 \mathrm{~g}$ IV over $15 \mathrm{~min}$ ]. Within $10 \mathrm{~min}$, his tachycardia, tachypnea, and dyspnea significantly improved, and he was admitted to the ICU. Three hours later, his bicarbonate was 22, anion gap 10, and lactate 5.6. Within $12 \mathrm{~h}$, his bicarbonate normalized to 24 , anion gap 9, and lactate was 1.0 with complete symptom resolution. He was discharged on day 3 .

Conclusions: This patient likely inhaled or ingested miniscule amounts of sodium cyanide from cleaning his inhaler with a jewelry cleaner known to contain that substance. Cyanide poisoning is a clinical diagnosis, requiring a high degree of suspicion. Anion gap metabolic acidosis with a high lactate, often $>10 \mathrm{mmol} / \mathrm{L}$, may be the only laboratory clue. Rapid treatment with an antidote such as hydroxocobalamin is key to preventing mortality. Physicians catering to diverse ethnic populations must be aware of their patients' cultural practices for early diagnosis and treatment of cyanide poisoning.

094. Life-Threatening Bufadienolide Toxicity after Eating a Toad Treated Successfully with Digoxin Immune Fab

Todd Phillips ${ }^{1}$, Garrett Taylor ${ }^{2}$, Michael Lynch $^{3}$

${ }^{1}$ JPS Health Network, Fort Worth, Texas, USA. ${ }^{2}$ University Of Oklahoma, Tulsa, Oklahoma, USA. ${ }^{3}$ University of Pittsburgh, Pittsburgh, Pennsylvania, USA

Background: Cardioactive steroids are found in the environment in many different forms and can cause digoxin-like toxicity. Several reports have shown toxicity associated with toad venom in the form of Chan $\mathrm{Su}$, a Chinese medicinal herb or directly from the toad itself. Little evidence has been found of successful treatment of potentially life threatening cardioactive steroid toxicity following ingestion of a toad with digoxin immune FAB.

Methods: A 29-year-old healthy male prison inmate was brought to the Emergency Department after ingesting the head of a toad on a bet. Three hours later, the patient became bradycardic with otherwise normal vital signs. An electrocardiogram showed sinus bradycardia at a rate of 40 beats per minute. Following medical toxicologist consultation, a digoxin level was obtained. Laboratory evaluation showed serum potassium of $6.9 \mathrm{mEq} / \mathrm{L}$ non-hemolyzed, white blood cell count of $23.5 \mathrm{~K} / \mathrm{mm}^{3}$, and serum digoxin level of $0.9 \mathrm{ng} / \mathrm{mL}$. Given the patient's detectable digoxin level and hyperkalemia, $400 \mathrm{mg}$ of digoxin immune FAB (ten vials) were administered. Forty-five minutes after administration, the patient's vital signs normalized and remained normal for the duration of his hospital stay. The patient was admitted to the ICU for close monitoring. Repeat digoxin level was $0.38 \mathrm{ng} / \mathrm{mL}$. 2D echocardiogram confirmed normal structure of the heart. The patient was discharged on hospital day 2 .

Discussion: Acute cardioactive steroid toxicity with a potassium of $6.9 \mathrm{mEq} / \mathrm{L}$ is associated with near $100 \%$ mortality without digoxin immune FAB reversal. Digoxin levels do not necessarily correlate with toxicity due to the limited cross-reactivity of the bufadienolide toxin with the digoxin immunoassay. Environmental sources of cardioactive steroids can result in fatal toxicity. 
Conclusion: While the serum assay may not reflect the degree of toxicity, digoxin immune FAB therapy should be considered in suspected severe cases of cardioactive steroid toxicity.

\section{Cardiac Toxicity After Trazodone Overdose}

Michael Rivara ${ }^{1}$, Dawn Pluckrose ${ }^{2}$, William Eggleston ${ }^{1,3}$

${ }^{1}$ SUNY Upstate Medical University, Department of Emergency Medicine, Syracuse, NY, USA. ${ }^{2}$ Albany College of Pharmacy and Health Sciences, Albany, NY, USA. ${ }^{3}$ Binghamton University School of Pharmacy and Pharmaceutical Sciences, Binghamton, NY, USA

Background: Trazodone is reported to cause conduction abnormalities and dysrhythmias. However, the incidence of cardiac toxicity in overdose is unknown. We report a retrospective review of trazodone overdoses with cardiac toxicity reported to a regional Poison Center.

Hypothesis: Trazodone causes QTc prolongation in overdose, but the risk for dysrhythmias is low.

Methods: We conducted a retrospective review of trazodone overdoses reported to a regional Poison Center between January 1, 2008 and December 31,2017 . A case was defined as an adult ( $\geq 18$ years of age) with trazodone overdose causing a conduction abnormality and a reason for exposure of intentional "abuse," "misuse," "suspected suicide," or "unknown." All cases were reviewed by two researchers. Demographics, dose, QRS/QTc, cardiac effects, and interventions were extracted.

Results: One hundred seventy-one cases were identified with a median age of 42 years (range 18-73), 33\% were male, and the median dose ingested was $1500 \mathrm{mg}(n=56$, range 100-4000). Co-ingestants were reported in $78 \%$ of cases. Patients had a median QRS of $92 \mathrm{~ms}(n=$ 141 , range 60-172) and a median QTc of $515 \mathrm{~ms}(n=170$, range 387647). In cases where trazodone was the lone substance $(n=36)$, patients had a median QRS of $90 \mathrm{~ms}(n=32$, range 76-112) and a median QTc of $512 \mathrm{~ms}(n=36,461-647)$. There were four cardiac arrests; however, these all involved co-ingestants. There were no cases of Torsades de Pointes. The most common interventions were fluids (82\%) and magne$\operatorname{sium}(78 \%)$.

Conclusion: Our results support the hypothesis that trazodone overdose causes QTc prolongation, but the risk of dysrhythmias is low. Although further studies are needed, it is reasonable to conclude that patients presenting with QTc prolongation after trazodone overdose are at low risk for dysrhythmias.

096. Severe Flecainide Toxicity with Cardiac Arrest Treated with $3 \%$ Hypertonic Saline in Addition to Standard Sodium Bicarbonate Therapy

Laura Tortora $^{1,2}$, Joshua Canning ${ }^{1,2}$

${ }^{1}$ Department of Medical Toxicology, Banner University Medical CenterPhoenix, Phoenix, AZ, USA. ${ }^{2}$ Division of Medical Toxicology and Precision Medicine, University of Arizona College of MedicinePhoenix, Phoenix, AZ, USA

Background: Flecainide is a class $1 \mathrm{C}$ antidysrhythmic that causes severe cardiotoxicity due to sodium and calcium channel blockade. While standard treatment centers around sodium bicarbonate $\left(\mathrm{NaHCO}_{3}\right)$ therapy, the use of $3 \%$ saline has not been well studied.

Methods: This is a single patient chart review. A 16-year-old boy was brought to ED in cardiac arrest. He had a witnessed seizure and became pulseless after ingesting approximately $2 \mathrm{~g}$ of flecainide. He was defibrillated a total of five times for ventricular tachycardia and one episode of torsades de pointes. He was intubated and received $1 \mathrm{~g} / \mathrm{kg}$ activated charcoal. EKG after ROSC demonstrated QRS $202 \mathrm{~ms}$ and $\mathrm{QT}_{\mathrm{c}}$ $461 \mathrm{~ms}$. After an initial five ampule $\mathrm{NaHCO}_{3}$ bolus, a $\mathrm{D}_{5} \mathrm{~W}$ infusion with $150 \mathrm{mEq} / \mathrm{L} \mathrm{NaHCO}$ was started.

Over the first $72 \mathrm{~h}$, any attempts to wean $\mathrm{NaHCO}_{3}$ infusion precipitated widened QRS, requiring boluses of both $3 \%$ saline and $\mathrm{NaHCO}_{3}$ to maintain goal $\mathrm{pH} 7.55$ and $\mathrm{Na}^{+} 155$. Patient developed refractory hypotension requiring epinephrine, and rhythms varied between normal sinus, intermittent junctional escape and sinus bradycardia.

Results: Flecainide level approximately $3.5 \mathrm{~h}$ post-ingestion was $1.94 \mathrm{mg} / \mathrm{L}$ rising to $2.02 \mathrm{mg} / \mathrm{L}$ on HD2. Over $103 \mathrm{~h}$, he received $4.05 \mathrm{~L}$ $3 \% \mathrm{Na}^{+}$in addition to $4134 \mathrm{mEq} \mathrm{NaHCO}$. Bicarbonate infusion was weaned on HD 5. He was extubated on HD 6 and discharged on HD 10. Discussion: Reports of flecainide toxicity focus on sodium bicarbonate treatment, despite evidence that urinary alkalosis may decrease elimination. In this case, the aggressive use of $3 \%$ hypertonic saline in addition to standard bicarbonate therapy may have helped overcome $\mathrm{Na}^{+}$channel blockade and have averted need for intravenous lipid therapy and ECMO. Conclusion: We present a case of severe flecainide toxicity with cardiac arrest successfully managed with large amounts of hypertonic saline in addition to standard $\mathrm{NaHCO}_{3}$ therapy.

097. Ventricular Dysrhythmia Cardiac Arrests in the Setting of Loperamide Withdrawal

Todd Phillips, David Bryant, Matthew Brewer JPS Health Network, Fort Worth, Texas, USA

Background: Ingestion of loperamide at high doses for recreational or analgesic purposes is common. Case reports have identified fatalities with high levels of loperamide, with most happening during abuse of the product. Cardiac dysrhythmias in the setting of opioid withdrawal and likely low drug levels have not been described.

Methods: This is a single patient chart review of a middle-aged female with a history of depression and chronic back pain. She presented to a county hospital after taking 72 loperamide $4 \mathrm{mg}$ tablets daily for the previous 4-6 months for relief of chronic pain. Two days before arrival, she stopped all loperamide use. She was brought in by EMS after observed seizure-like activity in which the patient was unresponsive and shaking. She was alert and at baseline on presentation, with symptoms of generalized myalgia and loose stools. The ECG was compared to a prior ECG and lengthening of QRS and QTc were noted, electrolytes were unremarkable. The patient was given sodium bicarbonate and magnesium sulfate and was admitted to the hospital. The patient experienced a ventricular dysrhythmia cardiac arrest the day after admission. The QTc was variable but widened to $630 \mathrm{~ms}$ prior to the initial cardiac arrest. A second ventricular dysrhythmia cardiac arrest occurred on day three of admission. A cardiac catheterization later performed found no stenosis.

Results: The patient presented after experiencing achiness and loose stools suggestive of withdrawal, 2 days after cessation of loperamide. With withdrawal-like symptoms present on arrival and the second ventricular arrhythmia arrest on four days after medication cessation, toxicity was considered but may be attributable to persistent potassium channel effects of chronic use.

Conclusion: The dysrhythmogenic effects of loperamide may persist days beyond cessation. In the setting of chronic loperamide use, even without clinical signs of toxicity, a screening ECG may be useful.

098. Trends in Snakebites Before vs. After Texas Tropical Storms and Hurricanes, 2000-2017

Joann Schulte ${ }^{1}$, Kurt Kleinschmidt ${ }^{2}$, Ashley Haynes ${ }^{2}$, Brett Roth ${ }^{2}$, Eric Smith $^{3}$, Anelle Menendez ${ }^{3}$

${ }^{1}$ Houston Health Department, Houston, Texas, USA. ${ }^{2}$ University of Texas Southwestern, Dallas, Texas, USA. ${ }^{3}$ Parkland Health and Hospital Systems, Dallas, Texas, USA

Background: Anecdotal media reports suggest snakebites increase after hurricanes immediately after landfall or during the recovery when debris clean-up is underway and flooding is present. For example, after Hurricane Harvey, several households called Texas poison control centers to report snakebites that occurred during and after the storm. The patterns of snakebites before vs. after hurricane landfalls are poorly studied. 
Hypothesis: Snakebites increase following tropical storms and hurricanes.

Methods: We reviewed retrospective surveillance data from the Texas Poison Control Network to examine patterns of snakebites reported before and after tropical storms/hurricanes making landfall between 2000 and 2017. We examined snakebite trends overall and for each storm/hurricane in the 30 days before and after landfall in two groups of counties: those designated for individual assistance by FEMA and all other Texas counties. In counties designated for individual assistance, families or households can receives funds. Typically, such counties suffer the worst wind and rain damage.

Results: Eleven named tropical storms/hurricanes struck Texas between 2000 and 2017; no individual assistance designations were made for two events and those data were discarded. During the 18-year period, 2000 snakebites were reported in all of Texas during the 30 days before and the 30 days after landfalls in 9 storms. In designated counties, $119(6 \%)$ bites occurred before the landfalls and $122(6 \%)$ after landfall. Designated counties were not statistically more likely to have snakebites reported in the 30 days after storm landfall for any of the nine storms or overall.

Conclusion: No evidence of increased numbers of snakebites was found in Texas counties designated for individual assistance in any storm or overall. Evacuations and delayed returns to damaged and destroyed residences might reduce the number of persons who might be bitten after these storms.

\section{Did Not See that Coming: a Monocled Cobra Envenomation in Michigan}

Mirjana Dimovska ${ }^{1}$, Diana Dean ${ }^{1}$, Cynthia Aaron ${ }^{2,1}$, Bram Dolcourt ${ }^{1,2}$, Andrew King ${ }^{1,2}$, Benjamin $\mathrm{Abo}^{3,4}$, Matthew Hedge $\mathrm{e}^{1,2}$

${ }^{1}$ Michigan Regional Poison Control Center, Detroit, MI, USA. ${ }^{2}$ Department of Emergency Medicine Wayne State University School of Medicine, Detroit, MI, USA. ${ }^{3}$ University of Florida, Gainesville, FL, USA. ${ }^{4}$ Miami-Dade Fire Rescue Venom Response Unit, Doral, FL, USA

Background: Monocled cobra venom is composed of a complex mixture of alpha neurotoxins, cytotoxins and cardiotoxins.

Hypothesis: Improved outcomes of exotic cobra envenomation are seen with administration of the most specific antivenom available.

Methods: This is a case report of a single patient envenomation by a Naja naja kaouthia.

Results: Twenty-six-year-old male was envenomated by his pet snake on his left ankle. Within $20 \mathrm{~min}$, he developed nausea, vomiting, and difficulty in breathing. Respiratory failure from neuromuscular paralysis was apparent upon ED arrival, requiring intubation and transfer to a tertiary care facility. VS at transfer were pulse 141 beats/min, blood pressure 209/ $109 \mathrm{mmHg}$, and respiratory rate 14 breaths $/ \mathrm{min}$. He was paralyzed and developed ARDS (A-a gradient 532). He received eight vials of SAIMR polyvalent antivenom $6 \mathrm{~h}$ post envenomation with a partial response resulting in axial muscle movement but continued fine motor, bulbar, and respiratory paralysis. Eighteen hours later, he received speciesspecific Thai Red Cross Cobra antivenom with significant neurologic recovery and halting of wound progression. Additional doses of the antivenom were administered 52 (3 vials) and 61 (4 vials) hours post envenomation due to recurrent paralysis, with resolution in symptoms. Patient's course was complicated by progressive renal failure requiring CRRT and worsening ARDS. He was transferred to an ECMO facility but never required cannulation, was extubated on HOD 25 and discharged to a rehab facility.

Discussion: ARDS and AKI are uncommon with Naja naja kaouthia envenomations. SAIMR is a genus but not species specific antivenom for the monocled cobra. Species-specific variation in venom composition leads to an incomplete response to genus specific antivenom.

Conclusion: Naja naja kaouthia envenomation results in paralysis, local tissue necrosis, ARDS, and acute renal failure. Only a partial response is seen with SAIMR and species-specific antivenom should be administered whenever possible.
100. Rhabdomyolysis with Significant CPK Elevation After Copperhead Envenomation in a Child

Kartik Shah, Ann-Jeannette Geib, Michael Beuhler

Carolinas Medical Center, Charlotte, NC, USA

Background: Severe rattlesnake envenomations have been associated with CPK elevations and rhabdomyolysis. However, copperhead (Agkistrodon contortrix) envenomations have not been reported in the literature to have significant CPK elevations.

Methods: Single patient chart review. A 7-year-old female, $33 \mathrm{~kg}$, sustained a copperhead envenomation to her right posterior distal Achilles tendon, with two fang marks. She received six vials total of Crotalidae polyvalent immune Fab in the first $24 \mathrm{~h}$ due to swelling, tachycardia, and severe pain.

Results: Her platelet count, fibrinogen, and coagulation studies were unremarkable during her admission. However, she had persistent, severe pain out of proportion to her exam needing IV opiates which progressed into her groin, as well as substantial swelling of her calf, so a CPK on day 3 was checked and resulted as 7518 IU. It rose to 10461 IU on day 4 and peaked at $14504 \mathrm{IU}$ on day 5. It declined to $8275 \mathrm{IU}$ on day 6 and to $256 \mathrm{IU}$ on day 10. Her leg compartments remained soft throughout her hospitalization, and her pain started improving before her CPK peaked. Her renal function was never impaired. Her ALT and AST on day 1 were $23 \mathrm{IU} / \mathrm{L}$ each, but they rose to $105 \mathrm{IU} / \mathrm{L}$ and $346 \mathrm{IU} / \mathrm{L}$ on day 3 , resp. On day 10, ALT was $145 \mathrm{IU} / \mathrm{L}$ and AST was $41 \mathrm{IU} / \mathrm{L}$.

Discussion: While her compartments remained soft, the venom likely spread into her superficial posterior compartment, leading to muscle breakdown and significant CPK elevation, explaining her severe pain as well as delaying her discharge. Her elevated transaminases were likely due to muscle breakdown as well.

Conclusion: Copperhead envenomations may cause significant CPK elevations with elevated transaminases if venom enters a large muscle compartment, leading to pain out of proportion to exam, rhabdomyolysis, and prolonged hospitalization.

101. Thromboelastography Demonstrating Coagulation Recovery Before Detection of Fibrinogen Level Following Rattlesnake Envenomation

Kartik Shah, Christine Murphy

Carolinas Medical Center, Charlotte, NC, USA

Background: Significant coagulopathy can occur following rattlesnake envenomation. Lab parameters used to monitor this include platelet count, fibrinogen, prothrombin time (PT), and INR. Thromboelastography (TEG) measures global clot formation properties of both primary and secondary hemostasis, along with fibrinolysis, but is not routinely used to manage envenomation related coagulopathy.

Hypothesis: TEG will quantify and monitor coagulation parameters more thoroughly than standard lab parameters.

Methods: In this single patient chart review, a 22 -year-old female sustained a rattlesnake bite with envenomation to her left foot. Her initial INR was 0.8 and platelets were $54,000 / \mu \mathrm{L}$. She received nine vials of Crotalidae polyvalent immune Fab and was discharged 5 days post-envenomation. She re-presented 11 days after envenomation for headache, foot pain, and systemic bruising without extracutaneous bleeding. Noncontrast CT head showed no intracranial hemorrhage. She underwent standard lab coagulation monitoring with subsequent TEG analysis.

Results: On re-presentation, her labs demonstrated INR $>13, \mathrm{PT}>100 \mathrm{~s}$, fibrinogen $<60 \mathrm{mg} / \mathrm{dL}$, and platelets $162,000 / \mu \mathrm{L}$. On day 14 , her INR was 3.9 , PT $38.7 \mathrm{~s}$, fibrinogen $<60 \mathrm{mg} / \mathrm{dL}$, and platelets $227,000 / \mu \mathrm{L}$; TEG showed R $8.2 \mathrm{~min}$, K not available, angle $19^{\circ}$, MA $10.5 \mathrm{~mm}$, and Ly30 67.2\%. On day 15 , her INR was 2.0 , PT $22.6 \mathrm{~s}$, fibrinogen $<60 \mathrm{mg} /$ $\mathrm{dL}$, and platelets $231,000 / \mu \mathrm{L}$; TEG showed R $6.4 \mathrm{~min}, \mathrm{~K} 7.6 \mathrm{~min}$, angle $34.2^{\circ}$, MA $24.4 \mathrm{~mm}$, and Ly30 51.6\%. On day 16, her INR was 1.5, PT 
$18.0 \mathrm{~s}$, fibrinogen $<60 \mathrm{mg} / \mathrm{dL}$, and platelets $243,000 / \mu \mathrm{L}$; TEG showed R $6.0 \mathrm{~min}, \mathrm{~K} 5.3 \mathrm{~min}$, angle $39.4^{\circ}$, MA $34.5 \mathrm{~mm}$, and Ly30 6.0\%. On day 17 , her fibrinogen was detectable $(65 \mathrm{mg} / \mathrm{dL})$.

Discussion: TEG demonstrated improving coagulation function, despite an undetectable fibrinogen level, providing evidence of physiologic improvement.

Conclusion: TEG analysis revealed restoration of coagulation function earlier than standard lab parameters.

\section{Cardiac Toxicity Secondary to Loxosceles reclusa Envenomation}

David Liss ${ }^{1}$, Caitlin McDowell ${ }^{2}$, William Dribben ${ }^{1}$

${ }^{1}$ Washington University, Saint Louis, Missouri. ${ }^{2}$ Roseville Medical Center, Roseville, California

Background: Cardiac toxicity is uncommon in cases of Loxosceles reclusa envenomation.

Methods: This is a single patient case report at a midwestern, urban, academic medical center. Data are obtained from direct patient care, the medical chart, and a novel enzyme-linked immunosorbent assay (ELISA) for $L$. reclusa venom.

Results: An 18-year-old male presented to the emergency department twice in 3 days with pleuritic chest pain and an erythematous lesion to his scapula. On his second to the emergency department, he presented with a diffuse rash. The wound on his scapula now had a bulla with ecchymosis and surrounding erythema. He was febrile, tachycardic, tachypneic, and hypotensive ( $\operatorname{Tmax} 38.1^{\circ} \mathrm{C}$, HR 128, RR 29, BP 84/ 54). His work-up was notable for C-reactive troponin $<0.03 \mathrm{ng} / \mathrm{mL}$ and urinalysis with $3+$ blood. Chest radiography was normal. A transthoracic echocardiogram was normal with an estimated ejection fraction of $67 \%$. An electrocardiogram showed sinus tachycardia with a heart rate of 120 beats $/ \mathrm{min}$. The patient was admitted to the intensive care unit. On hospital day 2, an electrocardiogram showed diffuse ST elevation and troponin was $2.11 \mathrm{ng} / \mathrm{mL}$. A repeat transthoracic echocardiogram showed an ejection fraction of $31 \%$. The patient's course was further complicated by respiratory failure requiring intubation and hemolytic anemia with a hemoglobin nadir of $7.6 \mathrm{~g} / \mathrm{dL}$. The patient's scapular wound eventually developed necrosis and ELISA testing confirmed Loxosceles reclusa venom. By hospital day 5, the patient was weaned from inotropes and extubated. A repeat echocardiogram showed an improved ejection fraction of 53\% and the patient was discharged on hospital day 9 .

Conclusion: We present a case of suspected systemic loxoscelism with a transient reduction in cardiac ejection fraction and elevation of serum troponin. Although unusual, cardiovascular compromise may be seen in patients with systemic loxoscelism.

103. Neurotoxic Envenomation by the Angolan Coral Snake, Aspidelaps lubricus cowlesi

Ryan Marino, David Barton, Shirley Shao, Anthony Scoccimarro, Alexander Sidlak, Joseph Yanta, Anthony Pizon

University of Pittsburgh School of Medicine, Pittsburgh, PA, USA

Background: The Elapidae family of snakes is known to have several species with neurotoxic venom. Aspidelaps lubricus cowlesi, the Angolan coral snake, is in this family, but has not previously been reported to cause neurotoxic envenomation in humans.

Hypothesis: Aspidelaps lubricus cowlesi has potential to inflict a lifethreatening envenomation.

Methods: This is a single patient case report. A 44-year-old man was bitten twice, once on each hand, by his captive Angolan coral snake while feeding a female who had recently laid eggs. Over an hour, he developed eyelid drooping, difficulty speaking, vomiting, inability to move his tongue, and dyspnea. His wife then activated EMS. Upon arrival to the Emergency Department, he was tachypneic (42 bpm) hypoxemic $\left(\mathrm{SpO}_{2}\right.$ $78 \%)$, and hypertensive $(198 / 121 \mathrm{mmHg})$. He was intubated with etomidate and succinylcholine for hypoxic respiratory failure and transferred to a tertiary center.

Results: Neurological examination revealed facial muscle paralysis, bilateral upper extremity paralysis, with preserved strength, and voluntary control of his lower extremities. He had puncture wounds in both hands without swelling, erythema, ecchymosis, or bullae. Total creatine phosphokinase level was normal. Laboratory studies showed no thrombocytopenia and no coagulopathy, with exception of mild d-dimer elevation $(1.72 \mu \mathrm{g} / \mathrm{mL}$ reference range, $0.00-0.49)$, which has been documented in reports of envenomation by other Elapids. Supportive care was provided, and paralysis and respiratory failure resolved spontaneously $12 \mathrm{~h}$ after onset.

Discussion: We report a case of envenomation by Aspidelaps lubricus cowlesi, the Angolan coral snake. Presenting symptoms of respiratory failure and upper body paralysis indicate neurotoxic venom component. This elapid species is very popular in private collections and there is no antivenom available. Physicians, first responders, and herpetologists should be aware of the potential for serious systemic envenoming by $A$. lubricus.

Conclusion: This is the first medically documented account of systemic envenoming by Aspidelaps lubricus cowlesi.

104. Complete Cardiovascular Collapse After Digoxin Immune Fab (Ovine) Reversal in Chronic Digoxin Toxicity

Ryan Marino, Nicholas LaFountain, Andrew Micciche, Matthew Charek, Joshua Shulman

University of Pittsburgh School of Medicine, Pittsburgh, PA, USA

Background: Digoxin is used to treat heart failure, a condition associated with high mortality and high incidence of sudden death. Not uncommonly, this population can develop supratherapeutic drug levels, which pose a clinical dilemma given a lack of consensus regarding treatment of chronic digoxin toxicity and role of Digoxin Immune Fab (ovine).

Hypothesis: Digoxin Immune Fab administration in chronic digoxin toxicity may unmask underlying heart disease causing hemodynamic decompensation.

Methods: This is a single patient case report. A 77-year-old woman on chronic digoxin therapy for biventricular heart failure (left ventricular ejection fraction $30 \%$ ) presented to the emergency department with rib pain after a fall in the setting of several days of nausea, vomiting, and generalized weakness. She was bradycardic $(57 \mathrm{bpm})$ and found to have two acute rib fractures and acute kidney injury (estimated glomerular filtration rate $31 \mathrm{~mL} / \mathrm{min})$. Digoxin $(4.3 \mathrm{ng} / \mathrm{mL})$ and potassium $(5.8 \mathrm{mMol} / \mathrm{L})$ levels were elevated. Patient was given semi-molar reversal with $40 \mathrm{mg}$ of Digoxin Immune Fab and admitted to the intensive care unit (ICU).

Results: Upon ICU admission, she maintained normal vital signs and repeat potassium was $4.7 \mathrm{mMol} / \mathrm{L}$. Six hours later, she received $80 \mathrm{mg}$ of additional Digoxin Immune Fab for complete molar reversal. Her blood pressure declined to $90 / 55 \mathrm{mmHg} 1 \mathrm{~h}$ after additional Digoxin Immune Fab, and $2 \mathrm{~h}$ later she was found with pulseless electrical activity. After 30 min of cardiopulmonary resuscitation, she was pronounced dead from suspected exacerbation of underlying structural heart disease.

Discussion: Supratherapeutic digoxin levels develop in patients with renal insufficiency. In underlying heart disease, especially with concurrent critical illness, abrupt removal of digoxin effect may lead to acute decompensation, and alternative strategies to optimize resuscitation should be considered first.

Conclusion: Reversal of chronic digoxin therapy may exacerbate underlying cardiac disease and precipitate cardiovascular collapse.

\section{Fomepizole Monotherapy for Massive Ethylene Glycol Ingestion}

Meghan Spyres, Fiona Garlich, Edwin Peck

University of Southern California, Los Angeles, CA, USA 
Background: Ethylene glycol toxicity can result in severe metabolic acidosis, renal failure, and death. Hemodialysis (HD) is typically employed as an adjunct to alcohol dehydrogenase inhibition in the setting of significant acidosis or end-organ toxicity. We present a case of EG toxicity with the highest reported level managed with fomepizole alone. Hypothesis: Patients with extremely high EG levels and acidosis experience complications if not treated with HD.

Methods: This is a single patient chart review

Results: A 39-year-old man presented to the ED with vomiting $2 \mathrm{~h}$ after ingesting $3 \mathrm{~L}$ of antifreeze. He was given $15 \mathrm{mg} / \mathrm{kg}$ of fomepizole and transferred to a regional toxicology center. Upon transfer, the patient was asymptomatic with pulse 79 beats/min, BP 109/72 mmHg, RR 19 breaths/min, T $36.7^{\circ} \mathrm{C}$, pulse oximetry $97 \%$ on room air. Initial laboratory values were significant for venous $\mathrm{pH} 7.36, \mathrm{HCO}_{3} 17 \mathrm{mEq} / \mathrm{L}, \mathrm{Cr}$ $1.18 \mathrm{mg} / \mathrm{dL}$, anion gap (AG) $13 \mathrm{mEq} / \mathrm{L}$, serum osm $461 \mathrm{mOsm}$, and osm gap (OG) 159. Fomepizole was continued every $12 \mathrm{~h}$ and adjunctive treatments including pyridoxine and thiamine were added. Three hours after arrival, his $\mathrm{pH}$ decreased to 7.28 with $\mathrm{HCO}_{3} 15 \mathrm{mEq} / \mathrm{L}$, and $5 \mathrm{~h}$ later his $\mathrm{HCO}_{3}$ had decreased to nadir of $11 \mathrm{mEq} / \mathrm{L}$. Hemodialysis was recommended but was held due to subsequent improving acidosis. Fomepizole was continued for 4 days, during which time his acidosis resolved and creatinine remained stable. Initial EG level resulted at $825 \mathrm{mg} / \mathrm{dL}$.

Conclusion: This patient with massive EG ingestion was treated successfully with fomepizole alone, despite the development of moderate acidosis. Fomepizole monotherapy has been described in patients with lower levels, mostly with normal renal function and no metabolic acidosis. The EG level of $825 \mathrm{mg} / \mathrm{dL}$ is, to our knowledge, the highest level reported in which fomepizole monotherapy was used.

\section{Octreotide Use by Medical Toxicologists: an Analysis from the ToxIC Registry}

Alexander Barbuto, Michael Chary, Michele Burns

Harvard Medical Toxicology at Boston Children's Hospital, Boston, MA, USA

Background: Octreotide is used to counteract hypoglycemia from insulin-secretagogue overdose. Theoretically, it could mitigate hypoglycemia due to excess insulin release from other causes. Its use in nonsulfonylurea anti-hyperglycemic poisonings has not been well-described. Methods: We reviewed the ToxIC registry to describe octreotide use. We excluded patients who were not hypoglycemic, had no exposure to an anti-hyperglycemic, or did not receive octreotide. We defined hypoglycemia as blood glucose less than $60 \mathrm{mg} / \mathrm{dL}$ or free-text stating hypoglycemia. The primary aim was to quantify the association between hypoglycemia and treatment with octreotide. The secondary aim was to describe octreotide use in patients without sulfonylurea ingestion.

Results: We identified 144 patients with hypoglycemia of 7577 entries in ToxIC. Hypoglycemia was seen with anti-hyperglycemic medication exposure (66 of 144), analgesics ( $n=13)$, ethanol $(n=12)$, opioids ( $n=11)$, sympathomimetics $(n=6)$, and cardiovascular medications $(n=6)$ most commonly. Octreotide was used in 43 of these 144 patients. There was no significant difference in mortality when octreotide was used in all-cause hypoglycemia (Fisher exact test, not-significant). We identified 154 patients who had an anti-hyperglycemic exposure of 7577 entries. Of these, 14 had multiple anti-hyperglycemics listed (11 patients with two agents, 2 with three, and 1 with four). Metformin $(n=57)$ was the most common antihyperglycemic agent. Sulfonylureas were coded in 67 patients, 34 of whom received octreotide. In the 87 patients with non-sulfonylurea anti-hyperglycemic exposures, octreotide was almost exclusively used in patients with insulin exposure $(n=6)$. One patient had "diabetic med unspecified."

In 16 patients who received octreotide without anti-hyperglycemic exposure, no pattern of use was established.

Conclusion: There is no significant difference in mortality with octreotide use in all-cause hypoglycemia in the ToxIC registry.
Octreotide is widely used by toxicologists for sulfonylurea poisonings. Of non-sulfonylurea anti-hyperglycemic poisonings, insulin exposure is the most common specified instance in which octreotide is given.

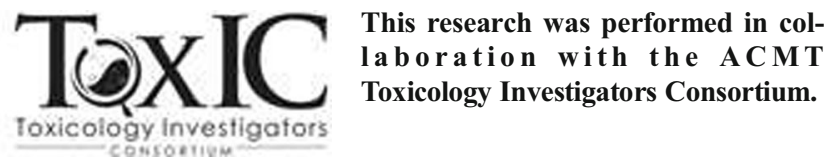

\section{The Cost of Toxic Alcohol Levels}

Nicholas Quinn $^{1}$, Anna Dulaney ${ }^{1,2}$, Michael Beuhler ${ }^{1,2}$

${ }^{1}$ Carolinas Medical Center, Charlotte, NC, USA. ${ }^{2}$ Carolinas Poison Center, Charlotte, NC, USA

Background: In December 2017, our hospital system stopped running ethylene glycol and methanol (toxic alcohol) levels, requiring analysis be performed outside of the system. The impact on antidotal use of an insystem toxic alcohol lab versus using send-out labs is unknown.

Hypothesis: Antidote usage will unnecessarily increase if in-system toxic alcohol lab values are not available.

Methods: This is a retrospective study of consecutive patients in one large hospital system who received fomepizole for presumed toxic alcohol poisoning. The Poison Center database (ToxiCALL $®)$ was used to identify patients from August 1, 2017 until April 30, 2018. Equal periods of 4.5 months were examined both before and after the lab became inoperable. Notes from the poison center cases as well as electronic medical records were reviewed to determine timing of toxic alcohol levels and fomepizole usage. Two-tailed Student's $t$ test was used to compare continuous variables.

Results: Thirty cases of fomepizole use were identified during our study period; 16 in the before period and 14 in the after period. The mean lab turnaround time was $7.5 \mathrm{~h}$ in the before period vs. $16.9 \mathrm{~h}$ in the after period $(p=0.001)$. This resulted in $14.3 \%$ of total fomepizole maintenance vials wasted on those with negative toxic alcohol levels in the before group versus $64.3 \%$ in the after group $(p=0.02)$. The 4 -month wasted cost on fomepizole to the hospital and patients was a difference of $\$ 16 \mathrm{~K}$ between groups; if the three outlier cases were included the cost increased to $\$ 35 \mathrm{~K}$. We did not evaluate the additional costs of administering co-factors, further laboratory testing, or length of stay.

Conclusion: This study demonstrates significant costs in time and money when toxic alcohol levels are not readily available. Having toxic alcohol laboratory analysis available in a large hospital system is recommended from an antidotal cost perspective.

\section{Day 3: Lightening Orals, Abstracts 108-114}

\section{Opioid-Associated Hearing Loss: a 20-Year Review from the NJ Poison Center}

Alex Mozeika ${ }^{1}$, Bruce Ruck ${ }^{2}$, Lewis Nelson ${ }^{3,2}$, Diane Calello ${ }^{2,3}$

${ }^{1}$ Rutgers New Jersey Medical School, Newark, NJ, USA. ${ }^{2}$ New Jersey Poison Information and Education System, Newark, NJ, USA. ${ }^{3}$ Department of Emergency Medicine, Rutgers New Jersey Medical School, Newark, NJ, USA

Background: Opioid-induced ototoxicity is a known complication of opioid exposure, although the mechanism remains unclear. While historically most closed linked to heroin and oxycodone, evolving reports suggest that it may be a class effect of opioids. However, the evidence is limited to case reports.

Research Question: This study aims to characterize opioid-induced hearing loss at a single Poison Control Center over a period of 20 years. Methods: A retrospective review was conducted using the NJ Poison Center records (ToxiCALL $®)$. Cases were identified using a priori free-text search terms including "deaf," "deafness," "hearing," and "sensorineural." Each identified case was included if both hearing loss 
and recent opioid exposure were reported. Cases were followed to hospital discharge.

Results: Forty-one cases were identified, mean age 29.4 years, $51.2 \%$ $(n=21)$ male. Reported heroin exposures comprised 51.2\% $(n=21), 18$ of which were heroin alone. The next most commonly cited opioids were oxycodone (seven cases), methadone (four cases), and tramadol (four cases). Hearing loss was described as tinnitus in $24.4 \%$ of cases, hypoacusis in $36.6 \%$ of cases, deafness in $29.3 \%$ of cases, and mixed tinnitus/hypoacusis in $9.8 \%$ of cases. Only $34.1 \%(n=14)$ of cases were associated with a potential hypoxic event. Although outcome was not documented in all cases, $36.6 \%(n=15)$ reported improvement.

Discussion: Opioid-induced ototoxicity is a hypoxia-independent adverse effect of a wide array of opioids, including tramadol. This may support an opioid receptor mediated effect, especially since most of the reported cases did not involve a known contributory hypoxic event. The ototoxic effect appears to be self-limited in many patients. This study is limited by the voluntary nature of spontaneous reporting to Poison Centers.

Conclusion: Opioid-associated ototoxicity was most commonly associated with heroin exposure and appeared independent of hypoxic events. Further investigation that clarifies the risk factors and long-term outcomes is needed.

109. Dose-Dependent Increased Risk of Adverse Cardiovascular Events and Mortality in Patients with Acute Drug Overdose Treated with Sodium Bicarbonate

Judson Ellis ${ }^{1}$, Rajesh Vedanthan ${ }^{2}$, Lynne Richardson ${ }^{3}$, Alex Manini ${ }^{3}$

${ }^{1}$ 1. Brown University Undergraduate School, Providence, RI, USA. ${ }^{2}$ New York University Langone School of Medicine, New York, NY, USA. ${ }^{3}$ Icahn School of Medicine at Mount Sinai, New York, NY, USA

Background: Sodium bicarbonate therapy (SBT) is provided in the emergency department (ED) for a variety of poisoning indications but its use is controversial. SBT administration may prolong the QTc interval and potentially cause increased rates of adverse cardiovascular events (ACVE). Hypothesis: SBT dose and duration will affect the risk of ACVE in an ED overdose patient population.

Methods: We prospectively analyzed consecutive ED patients with acute drug overdose who were given SBT at two urban teaching hospitals from 2015present. Data included SBT indication, dose, duration, and QTc (initial/peak from computer generated Bazett correction) during hospital stay. We used median values to dichotomize SBT total dose (high/low) and total duration (long/short). Patients were prospectively followed to hospital discharge for the occurrence of the primary outcome: ACVE and/or mortality. ACVE was defined as in-hospital ventricular dysrhythmia, myocardial infarction, shock requiring vasopressors, or cardiac arrest. Severe QTc prolongation was defined as $\geq 500 \mathrm{~ms}$. Ventricular dysrhythmias were adjudicated by a blinded cardiologist. Results: Indications for SBT in 30 patients analyzed were salicylism (5), sodium channel antagonist (6 TCA, 5 other), wide QRS in absence of known drug (9), acidosis or cardiac arrest (3), and unknown (2). After SBT, severe QTc prolongation occurred in $6(20 \%)$, ACVE in $17(57 \%)$, and $6(20 \%)$ died. There was a significant association between in-hospital severe QTc prolongation for both high dose and long duration groups $(p<.05)$ and SBT duration ( $100 \%$ long, $25 \%$ short, $p<.05)$ were significantly associated with the primary outcome.

Conclusion: Higher SBT doses and durations significantly increased the risk of ACVE in an ED overdose patient population. Severity of overdose may be an uncontrolled confounder. Overall, these results are consistent with the hypothesis that SBT may cause unintended ACVE.

110. Comparison of Outcomes Between Extracorporeal Elimination Techniques in Metformin Associated Lactic Acidosis Related to Chronic Metformin Use

Joshua Shulman, Anthony Scoccimarro, Ryan Marino, Alexander Sidlak, Michael Lynch, Anthony Pizon, Shirley Shao, Joseph Yanta, Michael Abesamis
University of Pittsburgh Division of Medical Toxicology, Pittsburgh, PA, USA

Background: Metformin-associated lactic acidosis (MALA) is a rare condition associated with metformin accumulation. With chronic use, metformin's volume of distribution increases due to erythrocyte sequestration. Intermittent hemodialysis (IHD) was historically preferred for clearance due to higher flow rates and more effective dialysis membrane. Continuous venovenous hemodialysis (CVVHD) is becoming more prevalent due to ease of operation and perceived benefit in hemodynamically unstable patients.

Hypothesis: IHD is associated with a mortality benefit compared to CVVHD in MALA patients with chronic metformin use.

Methods: Case series of 19 subjects between September 2015 and October 2018 with presumed diagnosis of MALA. Seven patients excluded (absent level in four, admission metformin undetectable in three). We performed descriptive statistics, Student's $t$ test, and $\chi^{2}$ test. Nine patients received intermittent hemodialysis (IHD) only, two patients had only CVVHD, and one received both IHD, then CVVHD (included in CVVHD group).

Results: Overall, eight subjects (62\%) were male, with mean age 70 years (52-87 years). All took between 1 and $2 \mathrm{~g}$ of metformin daily and were critically ill. Both groups had similar initial lactate level, peak lactate level, and creatinine elevations from baseline. Higher metformin levels on presentation were found in the CVVHD group $(p=0.01)$. Overall mortality was $42 \%(5 / 12)$, with $22 \%$ (2/9) mortality in the IHD group and $100 \%(3 / 3)$ mortality in the CVVHD group $(p=0.02)$.

Discussion: Limitations included small sample size and multifactorial confounders of morbidity, including renal failure, and sepsis. Metformin clearance could not be quantified across modalities due to inconsistent ability to obtain levels. Nevertheless, the higher rate of mortality in the CVVHD group could be secondary to ongoing mitochondrial dysfunction related to reduced metformin clearance.

Conclusion: Mortality after IHD is lower compared to those receiving CVVHD in MALA due to chronic metformin toxicity.

111. Rates of Serotonin Toxicity and Factors Associated with Seizures in SNRI Overdose: a Review of the Toxicology Investigators Consortium Database

Tony Rianprakaisang ${ }^{1,2}$, Colin Prather $^{1}$, Lauren Murphy ${ }^{1,2}$, Adam Blumenberg ${ }^{1,2}$, Adrienne Hughes ${ }^{1,2}$, Robert Hendrickson ${ }^{1,2}$

${ }^{1}$ Oregon Health and Science University, Portland, OR. ${ }^{2}$ Oregon Poison Center, Portland, OR

Background: SNRI's are known to cause seizures and serotonin toxicity in overdose.

Objective: To investigate factors associated with seizures in SNRI overdose and compare rates of seizures and serotonin toxicity between these medications.

Methods: We queried the ToxIC database for SNRI overdoses. Cases involving co-ingestion of other epileptogenic agents (bupropion, tramadol, citalopram, TCA's) were excluded. We selected a number of a priori clinical features and calculated their association with seizure using odds ratios. We also compared rates of serotonin toxicity between medications. Results: Rates of serotonin toxicity after overdose of venlafaxine, desvenlafaxine, and duloxetine were $15.6 \%, 16.3 \%$, and $15.1 \%$, respectively, with no statistically significant difference between them. Rate of seizures after overdose of venlafaxine $(11.5 \% ; 45 / 391)$ was higher than with duloxetine $(3.8 \% ; 8 / 204)(p=0.02)$, but not higher than with desvenlafaxine $(7 \% ; 3 / 43)(p=N S)$. Factors associated with seizures after venlafaxine overdose were tachycardia $>140$ BPM (OR 2.59, CI 1.30 5.12), QTc $>500 \mathrm{~ms}$ (OR 3.48, CI 1.17-10.39), and hypotension (OR 5.61, CI 2.05-15.3). There were no factors that were statistically significantly associated with seizures after duloxetine and desvenlafaxine overdose, however low seizure rates limited our analysis. 
Discussion: Tachycardia, QTc prolongation, and hypotension are associated with seizures in venlafaxine overdose. While rates of serotonin toxicity were consistent between groups, venlafaxine was associated with significantly higher rates of seizure than duloxetine. This finding suggests that duloxetine may be less epileptogenic than venlafaxine in overdose. Conclusion: Venlafaxine appears to cause seizures in greater frequency than duloxetine in overdose. Additionally, there are multiple clinical features associated with seizures in venlafaxine overdose.

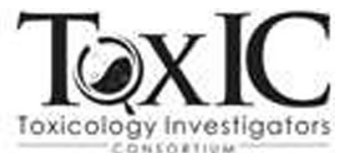

This research was performed in collaboration with the ACMT Toxicology Investigators Consortium.

\section{Utilizing the ToxIC Network to Assess the Impact of Opioid and Benzodiazepine Misuse/Abuse in Older Adults}

Meghan Spyres ${ }^{1}$, Maryann Mazer-Amirshahi ${ }^{2,3}$, Anne Riederer ${ }^{4}$, Sharan Campleman ${ }^{4}$, Paul Wax ${ }^{4}$, Jeffrey Brent ${ }^{4,5}$

${ }^{1}$ University of Southern California, Los Angeles, CA, USA. ${ }^{2}$ Georgetown University School of Medicine, Washington, DC, USA. ${ }^{3}$ MedStar Washington Hospital Center, Washington, DC, USA. ${ }^{4}$ ACMT, Phoenix, AZ, USA. ${ }^{5}$ University of Colorado School of Medicine, Denver, CO, USA

Background: Prescription opioid and benzodiazepine use among older adults (age $>50$ years) continues to rise as the population ages. Although policy changes aimed at reducing unsafe prescribing practices have been enacted, overdose and deaths involving prescription opioids continues to rise in older adults. Factors contributing to this trend remain unclear.

Hypothesis: Prescription opioid and benzodiazepine misuse/abuse is a major problem resulting in significant toxicity in older adults reported to the ToxIC registry.

Methods: Data reported to the ToxIC Database between January 2015 and December 2017 were reviewed. Inclusion criteria were age $>50$ years and first or second agent prescription benzodiazepine or opioid. Data collected included demographics, medical history, overdose characteristics, clinical outcomes, and treatment modalities.

Results: Nine hundred twenty-seven cases, with 16 confirmed deaths, were included; 180 misuse/abuse/self-harm cases were reported, including 97 single-agent opioid, 58 single-agent benzodiazepine, and 25 polypharmacy opioid/benzodiazepine cases. Eighty-five (47\%) were ages 50-59 years, $56(31 \%)$ were ages $60-69$ years, $23(12.8 \%)$ were ages $>$ 70 years, $16(8.9 \%)$ were age unspecified $>50$. Eighty-eight $(48.9 \%)$ were female. CNS depression was the most common clinical symptom (127 (70.5\%)), followed by respiratory depression (58 (32.2\%)). Major vital signs abnormalities were reported in $58(30.6 \%)$ cases. Naloxone was administered in 75 (61.4\%) of opioid or combination overdoses. The top three single agent opioid ingestions were oxycodone, tramadol and methadone. Alprazolam, followed by clonazepam and lorazepam were the most common benzodiazepine ingestions. Sixteen deaths were reported.

Conclusion: Older adults with opioid and benzodiazepine misuse, abuse and self-harm reported to the ToxIC registry suffer significant clinical toxicity including CNS depression, respiratory depression, and vital sign abnormalities. More detailed data describing prescription characteristics, use patterns, clinical complications, and resource utilization from a subregistry is needed.

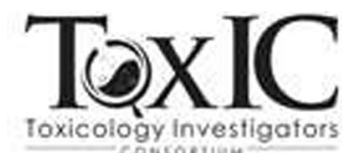

This research was performed in collaboration with the ACMT Toxicology Investigators Consortium.
113. Global Educational Toxicology Toolkit (GETKIT): 1-Day Course in Poisoning Essentials in Low- and Middle-Income Countries: Development and Pilot Data

Kathryn Kopec ${ }^{1}$, Rais Vohra ${ }^{2,3}$, Cynthia Santos ${ }^{4}$, Ziad Kazzi $^{5}$, Anselm Wong 6,7

${ }^{1}$ Carolinas Medical Center, Charlotte, NC, USA. ${ }^{2}$ California Poison Control System, Fresno, CA, USA. ${ }^{3}$ UCSF Fresno Medical Center, Fresno, CA, USA. ${ }^{4}$ Rutgers University, Newark, NJ, USA. ${ }^{5}$ Emory University, Atlanta, GA, USA. ${ }^{6}$ Victorian Poisons Information Centre \& Austin Toxicology Service, Victoria, Australia. ${ }^{7}$ Austin Hospital \& School of Clinical Sciences at Monash Health, Monash University, Victoria, Australia

Background: Worldwide an estimated one million deaths occur annually as a result of poisoning. Internationally, there is a lack of toxicology training programs, especially in resource poor countries. We hypothesized that the delivery of a one-day, interactive toxicology curriculum to healthcare practitioners in countries lacking clinical toxicology training is feasible and effective for knowledge dissemination.

Methods: GETKIT was developed with three sections: didactics, hands on toxicology case lab, and technology clinic. The investigators, who are medical toxicologists, created 21 didactic lectures and 42 workshop cases. Lectures and cases were peer reviewed by five senior toxicologists for content validity. Participants at pilot sites were given pre-course, postcourse, and 3-month follow-up tests and surveys.

Results: GETKIT was delivered at seven sites between November 2017 and April 2018. There were 186 total participants, from Kathmandu, Nepal; Puebla, Mexico; Mexico City, Mexico; Moshi, Tanzania; Dar es Salaam, Tanzania; Kotokal, India; and Bhubaneswar, India. One hundred and ten participants $(59 \%)$ reported their hospital lacked a toxicology service. The median post course score $12(60 \%)$, IQR $(6,14)$ was significantly higher compared to the pre-course score 9 (45\%), IQR (6, 11) $(p<0.0001)$. There was a significantly higher median 3 -month post course score $13(65 \%)$, IQR $(8,14)$ versus a median pre course score of 9 $(45 \%), \operatorname{IQR}(6,11)(p=0.0005)$. Three-month follow up surveys demonstrated median scores of 4 (scale 1-5) in regard to participants' use of the information obtained from GETKIT course, improved clinical management, and use of resources obtained from the technology clinic. At 3month follow up, $86 \%$ of participants reported GETKIT had changed their clinical practice.

Conclusion: We demonstrated an improvement in and retention of medical toxicology knowledge with the one-day GETKIT course. It also demonstrated that it could change poisoning management practices in participants from low resource settings.

\section{Clinical Predictors of Adverse Cardiovascular Events for Acute Pediatric Drug Exposures}

Stephanie Carreiro ${ }^{1}$, Simone Miller ${ }^{1}$, Bo Wang ${ }^{1}$, Paul Wax ${ }^{2}$, Sharan Campleman $^{2}$, Alex Manini ${ }^{3}$

${ }^{1}$ University of Massachusetts Medical School, Worcester, MA, USA.

${ }^{2}$ American College of Medication Toxicology, Phoenix, AZ, USA.

${ }^{3}$ The Icahn School of Medicine at Mount Sinai, New York, NY, USA

Background: Adverse cardiovascular events (ACVEs) following drug exposures have been well-characterized in adults but not adequately studied in children.

Research Question: What is the incidence and characteristics associated with ACVEs among children after drug exposure?

Methods: A prospective cohort of pediatric patients (age $<18$ ) was extracted from the Toxicology Investigators Consortium Registry for the period of 2010 and 2016. The primary outcome was ACVE (myocardial injury, hypotension, ventricular dysrhythmia, or cardiac arrest) The secondary outcome was death. Logistic regression models were fitted using stepwise variable selection, with calculation of odds ratios (ORs) and 
95\% confidence intervals (CIs). A logistic regression model was fitted to replicate a previously validated adult ACVE model.

Results: Of 13,097 patients (58.5\% female), there were 278 ACVEs $(2.1 \%)$ and 39 deaths $(0.3 \%)$. Drug classes significantly associated with $\operatorname{ACVE}(p<0.05)$ were cardiovascular agents, antidepressants, non-opioid analgesics, and opioids. Opioids were significantly associated with death $(p<0.05)$. On multiple logistic regression, age, and type of exposure remained independently associated with ACVE. Compared to teens, children under 2 (OR 0.41, CI 0.21-0.80), ages 2-6 (OR 0.356, CI 0.20 0.68 ), and ages $7-12$ (OR 0.48 , CI $0.26-0.91$ ) were less likely to experience ACVE. Odds ratios for ACVE with exposure to opioids and cardiovascular agents were 3.65 (CI 2.40-5.55) and 4.84 (CI 3.41-6.81) respectively. Presence of a bicarbonate $<20 \mathrm{mEq} / \mathrm{L}$ (OR 2.31, CI 1.48-3.60) or a QTc $>500 \mathrm{~ms}$ (OR 2.83, CI 1.67-4.79) were independently associated with ACVE.

Discussion: We successfully extrapolated previously derived predictors of ACVE from adults to a pediatric population of drug exposures.

Conclusion: Clinical predictors of ACVE for acute pediatric drug exposures are QTc $>500 \mathrm{~ms}$, bicarbonate $<20 \mathrm{mEq} / \mathrm{L}$, opioids, and cardiovascular drug exposures. ACVEs after pediatric drug exposures are associated with age and high risk drug classes.

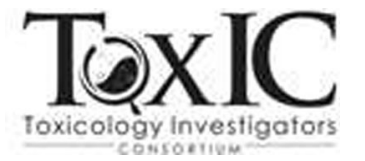

This research was performed in collaboration with the ACMT Toxicology Investigators Consortium.

\section{Day 3: Moderated Posters, Abstracts 115-120}

115. A Comprehensive Review of Consumer and Occupational Methylene Chloride Fatalities in the US from 1980 to 2018 and Policy Implications

Annie Hoang ${ }^{1}$, Kathleen Fagan ${ }^{2}$, Robert Harrison ${ }^{1}$, Dennis Shusterman ${ }^{1}$, Danielle Fries ${ }^{1}$, Veena Singla ${ }^{1}$

${ }^{1}$ University of California, San Francisco, San Francisco, CA, USA.

${ }^{2}$ OSHA, Washington DC, USA

Background: Methylene chloride is an organic solvent widely found in common consumer and industrial products including paint strippers, metal cleaners, degreasing agents, adhesives, and spray paints. In 2012, the Centers for Disease Control and Prevention reported that methylene chloride containing paint strippers were responsible for 13 fatalities among professional bathtub refinishers. However, there is no comprehensive identification and analysis of consumer or occupational methylene chloride fatalities in the USA.

Research Question: What are the incidents, circumstances, and demographic patterns of methylene chloride fatalities in the USA, and how have federal hazard communication, worker protection, and other policies influenced fatality patterns?

Methods: Mortality data were tabulated from surveillance systems, case reports in the peer-reviewed literature, legal databases, news reports, and government sources. We performed systematic searches in eight databases including PubMed and Lexis Nexis. Additionally, we requested data from the American Association of Poison Control Centers and the US Consumer Product Safety Commission. Fatality incidents were recorded with case report details in REDCap, with data QA/QC performed by an independent reviewer. SAS statistical software was used for data analysis.

Results: We identified and analyzed 83 methylene chloride related fatalities in the USA during the period 1980-2018. Of the cases with demographic information available, about $80 \%$ were male, with a median age of 31 years old. Paint stripping products caused the vast majority of fatalities, followed by adhesives and one pesticide case. Examination of reported occurrence by year revealed that federal regulatory actions did not have a clear impact on fatality patterns.
Conclusions: Comprehensive case finding and review of data on methylene chloride fatalities revealed a persistent pattern of mortality related to use in occupational and consumer settings. A policy approach focused on hazard elimination and safer substitutes would be more effective in addressing methylene chloride risks compared to the current reliance on hazard communication and administrative controls.

116. Urinary Gadolinium Levels After Contrast Enhanced MRI in Individuals with Normal Renal Function

Dalia Alwasiyah $^{1,2}$, Christine Murphy ${ }^{1,3}$, Paul Janetto ${ }^{4}$, Melanie Hogg ${ }^{1}$, Michael Beuhler ${ }^{1,3}$

${ }^{1}$ Atrium Health, Charlotte, NC, USA. ${ }^{2}$ King Faisal Research Center and Specialist Hospital, Riyadh, Saudi Arabia. ${ }^{3}$ Carolinas Poison Center, Charlotte, NC, USA. ${ }^{4}$ Mayo Medical Laboratories, Rochester, MN, USA

Background: Gadolinium-based contrast agents (GBCA) have been used to enhance magnetic resonance imaging (MRI) since 1985. Recently, there have been concerns voiced in the media and online groups about gadolinium deposition in patients with normal renal function based on "elevated" urinary gadolinium levels. The determination of increased urinary gadolinium levels is based on reference ranges developed in individuals with normal renal function who were never exposed to GBCA. Hypothesis: We hypothesize that 24-h urinary gadolinium concentrations in patients exposed to GBCA will exceed the current reference range of < $0.7 \mu \mathrm{g} / 24 \mathrm{~h}$ and that it will remain elevated for at least 30 days in most subjects.

Methods: This is a prospective observational pilot study. Subjects were between the ages of 18 and 65 without history of renal dysfunction who received GBCA for the first time. Urinary gadolinium was measured at days 3,10 , and 30 after GBCA exposure. We compared urinary gadolinium levels after GBCA exposure to the current reference range and calculated an estimated duration of "elevated" gadolinium urine levels in the average patient.

Results: All 13 subjects had $24 \mathrm{~h}$ urinary gadolinium levels higher than $0.7 \mu \mathrm{g} / 24 \mathrm{~h}$ with a mean of $1944( \pm 1432) \mu \mathrm{g} / 24 \mathrm{~h}$ on day $3,301( \pm$ 218) $\mu \mathrm{g} / 24 \mathrm{~h}$ on day 10 , and $34( \pm 33) \mu \mathrm{g} / 24 \mathrm{~h}$ on day 30 . Duration estimates for urinary levels remaining "elevated" exceeded 50 days.

Conclusion: The current reference range for $24 \mathrm{~h}$ urinary gadolinium is not applicable to patients for at least a month following GBCA exposure.

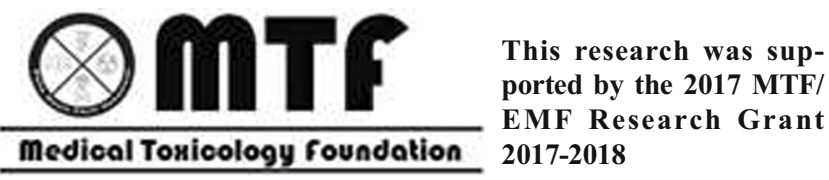

117. Hydrofluoric Acid Ingestions as Reported to the National Poison Data System, 2007-2017: Preliminary Findings

Brian Murray, Emily Kiernan, Joseph Carpenter, Camille Dunkley, Mark R Layer, Stephanie Hon, Sukhshant K Atti, Tim Moran, Robert Geller, Alaina Steck

Emory University School of Medicine, Atlanta, GA, USA

Background: Hydrofluoric acid (HF) ingestions are classically thought of as invariably fatal. However, aside from case reports, we are only aware of one published retrospective study that reported a $2 \%$ fatality rate and $9 \%$ systemic toxicity rate. These results appear to be in direct contradiction to the traditional belief about the risks associated with HF ingestions.

Methods: This is a retrospective chart review of HF ingestions reported to the National Poison Data System (NPDS) between 2007 and 2017. We describe the patient demographics, intent, signs and symptoms, treatment modalities, and case outcomes. A logistic mixed model regression was performed to determine the effect of route of exposure on the severity of patient outcome. 
Results: A total of 7740 total HF exposures and $893 \mathrm{HF}$ ingestions were recorded in the NPDS during the study period. We excluded 239 cases due to non-exposure or no known outcome, leaving 654 ingestions included for analysis. On average, there were $58.2(s=6.3)$ ingestions per year. Patients were $72.8 \%$ male and had a median age of 30 years (IQR = 11.3-46.8). Eighty-seven cases (13.6\%) involved multiple agents. Unintentional exposures accounted for $590(92.2 \%)$ cases and suspected suicide another $23(3.6 \%)$ cases. Five percent developed electrolyte abnormalities and less than $2 \%$ a dysrhythmia. Calcium was administered in $32.8 \%$ cases. There were 17 deaths $(2.6 \%)$. Significant health effects and death were more common for patients who ingested $\mathrm{HF}(7.4 \%$; $95 \% \mathrm{CI}$ $5.9-9.4 \%)$ than non-ingestion exposures $(2.4 \% ; 95 \%$ CI $1.9-2.9 \%$; OR = $4.5,95 \%$ CI 2.99-6.78, $p<0.001)$. This association remained significant after adjusting for confounders $(\mathrm{OR}=2.68 ; 95 \%$ CI $1.47-4.86, p=$ $0.001)$.

Conclusion: The morbidity and mortality associated with HF ingestions are lower than expected in this large NPDS sample; however, this data might be skewed by a high number of minor exposures. Ongoing analysis will evaluate case narratives to stratify ingestions by significance.

\section{Screening for Risk of Opioid Misuse and Overuse by Medical Toxicologists}

Sharan Campleman ${ }^{1}$, Paul Wax ${ }^{1,2}$, Anthony Pizon ${ }^{3}$, Dana Karshenas ${ }^{1}$, Jeffrey Brent ${ }^{4}$

${ }^{1}$ American College of Medical Toxicology, Phoenix, AZ, USA.

${ }^{2}$ University of Texas Southwestern Medical School, Dallas, TX, USA.

${ }^{3}$ University of Pittsburgh School of Medicine, Pittsburgh, PA, USA.

${ }^{4}$ University of Colorado School of Medicine, Denver, CO, USA

Background: Opioid use and overdose deaths represent a significant public health issue, which has reached epidemic proportions. Those with high-risk opioid use have higher mortality than their matched counterparts.

Research Question: To investigate the proportion of patients aged 12 years or older who were screened for the potential risk of opioid misuse/overuse during medical toxicology assessments.

Methods: Data was collected as part of the Centers for Medicare and Medicaid Services (CMS) approved ToxIC Qualified Clinical Data Registry (QCDR), a component of the ACMT ToxIC Registry. Participants were asked to report on whether their patients were screened for the potential risk of opioid misuse/overuse as part of their evaluation. The screening for opioid misuse/overuse could have been performed by any of the patient's health care providers including the medical toxicologist during the patient's evaluation. The denominator was all patients aged 12 years or older who had a toxicological consultation. The numerator was patients who were screened with a standardized tool (e.g., DAST, ASSIST), or assessed for the presence of an opioid abuse risk factors (e.g., survived an opioid overdose, taking more opioid than prescribed), or other screening method.

Results: During the first 6 months of data collection, data was submitted from 11 sites. During this period, 467 patients met the denominator. Twenty-three of these had incomplete data. One hundred eighty-seven were screened for opioid abuse/overuse. Of these 187, 9 used a standardized tool, 57 assessed for specific risk factors, and 121 used some "other" method to screen for opioid misuse/overuse. The performance rate that met the metric was $42.1 \%$.

Conclusion: Medical toxicologists do not use standardized screens to assess for the potential risk of opioid misuse/overuse. In more than $50 \%$ of cases, opioid misuse/overuse screening is not currently performed.

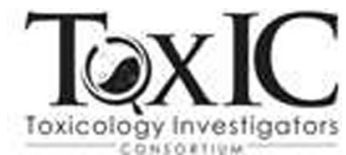

This research was performed in collaboration with the ACMT Toxicology Investigators Consortium.

\section{JMT's Preliminary Research Section-a 5-Year Evaluation}

David Jang ${ }^{1}$, Jennifer Love ${ }^{1}$, Mark Mycyk ${ }^{2}$

${ }^{1}$ Department of Emergency Medicine, Perelman School of Medicine, University of Pennsylvania, Philadelphia, PA, USA. ${ }^{2}$ Department of Emergency Medicine, Cook County Health, Chicago, IL, USA

Background: The JMT Preliminary Research: Research Concepts section was developed in 2010 to allow investigators to advance their research quickly. The goals of this section are threefold: (1) to provide a forum for rapid publication of peer-reviewed research, (2) to obtain high level reviews from established funded investigators, and (3) to support grant applications for extramural funding.

Objective: To examine the manuscripts published in the JMT Research Concepts section in the last five years and describe whether extramural funding followed their publication.

Methods: This was a retrospective evaluation of all submissions to the JMT Research Concepts section from 2014 to 2018. Data abstracted included dates of publication, study topic, and institutional affiliation. First authors were contacted to determine if awarded grant funding was related to their published manuscript because the manuscript was referenced in the grant submission in any way. All forms of extramural funding were included in our analysis.

Results: The JMT Research Concepts section published eight articles during this time period: each was reviewed by a minimum of two previously-funded peer reviewers, first decision to authors averaged 14.1 days, six required one revision and two required multiple revisions before publication. First-author institution included: University of Massachusetts (five manuscripts), University of Pennsylvania (two), and Iowa State University (one). Five papers focused on the integration of technology and toxicology, while three papers focused on basic science research. Multiple grants were funded related to the accepted manuscripts: two National Institutes of Health (NIH) career development awards (K08 and K23), acceptance into an institutional KL2 program, and an NIH R21 CounterACT grant.

Conclusions: The JMT Research Concepts section provides a novel forum for rapid review and publication of preliminary research. Published authors credit this section as a contributing factor to their successful grant applications.

120. Unintentional Ingestions of Beta-Antagonist in Pediatric Patients: a 10-Year Poison Center Review

Robert Goodnough ${ }^{1,2}$, Tom Kearney ${ }^{1,2}$, Craig Smollin ${ }^{1,2}$

${ }^{1}$ University of California San Francisco, San Francisco, California, USA.

${ }^{2}$ California Poison Control System - San Francisco Division, San Francisco, California, USA

Background: The incidence of significant clinical effect in unintentional ingestions of beta-antagonists is conflicting, despite documented lifethreatening hemodynamic, hypoglycemic, and central nervous system (CNS) toxicity in overdose and therapeutic dosing.

Hypothesis: Unintentional ingestions of beta-antagonist medications in patients under 6 years old are not associated with a high incidence of major clinical effects.

Methods: This is a retrospective review of hospitalized patients under the age of 6 years over a 10-year period (2008-2018), reported to the California Poison Control System. We included all accidental single substance ingestions of beta-antagonist medications. Chart coding was used to determine the incidence of symptoms (no clinical effect, minor, moderate, major) and death. All cases coded with clinical effect were manually abstracted by a single reviewer to determine incidence of clinical symptoms and the need for intervention.

Results: There were 1122 unintentional exposures to beta-antagonist medications, with 53 coded with clinical effect. Six non-accidental exposures were excluded. There were no deaths. The incidence of any clinical 
effect was $4.2 \%$ (47 cases). The incidence of CNS symptoms, gastrointestinal (GI) symptoms, hypotension, and bradycardia was $1.2 \%, 1.2 \%$, $1.3 \%$, and $1.2 \%$, respectively. No hypoglycemia was reported. The incidence of intravenous fluid and glucagon administration was $0.63 \%$ and $0.36 \%$, respectively. There was a non-significant trend toward clinical effect from propranolol (OR 3.12, 95\% CI 0.74-13.19), timolol (OR 3.24, 95\% CI 0.18-57.66), nebivolol (OR 1.1, 95\% CI 0.07-17.24), and sotalol (OR 1.66, 95\% CI 0.01-189.85).

Discussion: Symptoms in accidental ingestions of beta-antagonists in patients under 6 years old are rare. CNS, GI, and hemodynamic effects are equally likely. Hypoglycemia is unlikely. The low incidence of symptoms limited statistical power to differentiate risk by agent.

Conclusion: The unlikely need for intervention may limit invasive interventions or monitoring in pediatric patients, though propranolol, timolol, nebivolol, and sotalol likely warrant greater caution.

\section{Day 3: Posters, Abstracts 121-162}

\section{Lisinopril-Associated Hypoglycemia in a Non-Diabetic Patient}

Ashley Carter Powell ${ }^{1,2}$, Alex Chen ${ }^{1,2}$, Steven Curry ${ }^{1,2}$

${ }^{1}$ Department of Medical Toxicology, Banner - University Medical Center Phoenix, Phoenix, Arizona, USA. ${ }^{2}$ Division of Medical Toxicology and Precision Medicine, University of Arizona College of Medicine Phoenix, Phoenix, Arizona, USA

Background: Angiotensin-converting enzyme inhibitors (ACEIs) are commonly used antihypertensives associated with reports of druginduced hypoglycemia, primarily in diabetic patients taking enalapril or captopril. The frequency of this phenomenon and its mechanism are not well-understood.

Methods: Case report: A non-diabetic 76-year-old woman presented with recurrent symptomatic hypoglycemia over 3 weeks requiring four hospitalizations. Medications at the time included furosemide, lisinopril, meloxicam, omeprazole, and potassium chloride. Hypoglycemia persisted despite discontinuation of meloxicam. Hypoglycemia occurred daily, requiring oral or IV glucose supplementation. An extensive work up was performed in search of an endocrine cause of hypoglycemia. On consultation, medical toxicologists found that lisinopril was the only medication with reported association with hypoglycemia. Furthermore, she reported an increase in lisinopril dose from 10 to $20 \mathrm{mg}$ per day 1 week prior to her first hypoglycemic episode. Lisinopril had been continued at the higher dose during all episodes of hypoglycemia.

Results: Hypoglycemia during a fast was accompanied by low insulin and C-peptide levels, and normal growth hormone levels. Imaging studies and a cosyntropin stimulation test were normal. An acylcarnitine profile was normal. A sulfonylurea/meglitinide screen was negative and pill bottles contained the correct medications. Her lisinopril was discontinued and replaced with losartan, and she experienced no hypoglycemic episodes in the $24 \mathrm{~h}$ following discontinuation before discharge home. She was followed up over the next week by telephone and reported no further hypoglycemic episodes.

Discussion: This case strongly supports a causal association between lisinopril and hypoglycemia. The limited literature available suggests hypoglycemia may result from increased insulin receptor sensitivity in peripheral tissues, though impaired gluconeogenesis cannot be completely excluded.

Conclusion: ACEI-induced hypoglycemia should be considered in a patient with unexplained low blood glucose concentrations.

122. Optimizing Medication Safety for Behavioral Health Patients Boarding in the Emergency Department

Joanne Routsolias, Trevor Lewis, Mark Mycyk

Cook County Health, Chicago, IL, USA

Background: Previous study demonstrated medication errors are frequent in behavioral health patients with prolonged ED boarding times.
Since boarding has increased nationally, we performed a needs assessment study to determine how pharmacy-led initiatives may improve outcomes in these patients.

Methods: A closed-format survey instrument was developed by experts in operations, behavioral health, and safety. The instrument was piloted, revised, then administered electronically over a two week period until targeted participation reached $N=100$. Eligible subjects were all ED staff at an urban academic hospital with > 130,000 annual visits; participation was voluntary, anonymous, confidential, and approved by our IRB. Data collected included demographics and staff perceptions about behavioral health patients and ED boarding.

Results: Out of 100 subjects, 26 were attending physicians, 17 resident physicians, 39 nurses, 4 physician assistants, 10 ER technicians, and 4 others. Most (99\%) felt behavioral health patients often board in the ED for $>24 \mathrm{~h}$. The majority $(88 \%$ ) reported the physician is not always aware of medication needs of patients: $90 \%$ reported patients did not receive their usual non-psychiatric medications (e.g., diabetes, hypertension) while boarding, and $88 \%$ reported patients did not receive their psychiatric medications. Most (96\%) agreed daily clinical pharmacist rounds would improve medication safety, and $86 \%$ agreed a daily safety huddle involving all health care providers led by pharmacy would improve the needs of patients. When asked if boarding would ever improve, $21 \%$ reported yes, $54 \%$ were unsure, and $25 \%$ said no.

Conclusion: Our needs assessment confirms ED staff perceive behavioral patients with prolonged ED boarding are at risk for medication errors. A multidisciplinary approach led by pharmacy that performs comprehensive medication management and reconciliation should be implemented to improve medication safety and outcomes in patients with prolonged ED boarding. Enhanced clinical pharmacy integration in the ED would meet an ACMT priority.

123. Adverse Events Related to the Administration of Intravenous Hydralazine for the Management of Hypertension in Non-ICU Settings

Gerald Maloney

Louis Stokes Cleveland VA Medical Center, Cleveland, OH, USA. Case Western Reserve University, Cleveland, OH, USA

Background: IV hydralazine is a vasodilator frequently used as a parenteral agent for the management of hypertension in hospitalized patients. However, as it is used as bolus agent and is not titratable, there is a risk of hypotension.

Hypothesis: Treatment of hypertension using intravenous hydralazine may pose a risk for symptomatic hypotension.

Methods: All charts of patients receiving intravenous hydralazine between September 1, 2016 and September 30, 2017 were reviewed. Charts of patients in the ED, ICU, and PACU were excluded. There were no obstetrical patients. Data included systolic and diastolic blood pressure readings pre- and post-treatment, heart rate pre- and post-treatment, and any symptoms related to hypertension. The primary author reviewed all charts.

Results: Fifty-four patients received at least 1 dose of intravenous hydralazine, with a mean dose of $13.5 \mathrm{mg}$ (range, 10-20), with 14/54 receiving a second dose of hydralazine and 18/54 receiving an additional antihypertensive (metoprolol IV). Then, 48/54 received at least one oral antihypertensive as well prior to any IV medications. The mean SBP was $189 \mathrm{mmHg}$ and DBP $103 \mathrm{mmHg}$ pretreatment. In 1/54 patients, chest pain was documented; $5 / 54$ were NPO and could not take oral medications. In the remaining 49, there were no other indications for IV antihypertensives. Mean SBP posttreatment was $154 \mathrm{mmHg}$ (range, 74-189) and DBP $76 \mathrm{mmHg}$ (range, 40-111). Further, 4/54 required admission to a higher level of care due to hypotension, with $3 / 4$ receiving 2 doses of IV medication.

Conclusion: IV hydralazine is commonly used for treatment of hypertension. Significant hypotension from hydralazine alone was rare. The 
majority of patients treated did not have an indication for acute blood pressure lowering with IV medications, indicating a need for better education of the inpatient providers.

\section{Lichenoid Drug Reaction After Induction of Antihypertensive}

Kim Kwai, Jonathan Ford

University of California, Davis, Sacramento, CA, USA

Background: We describe a patient with a lichenoid drug eruption shortly after starting lisinopril.

Methods: This is a single case report of a lichenoid drug eruption in a 55year-old male shortly after starting lisinopril for hypertension. His past medical history also included type 2 diabetes mellitus. He presented to the emergency department with 1 week of a pruritic rash starting on his feet and spreading to his medial thighs, buttocks, back, and arms. He denied any systemic symptoms. About 1.5 weeks prior, his doctor changed his hypertension treatment from atenolol to lisinopril. He had no known allergies, no new soaps/detergents, no additional new medications, no history of camping or travel, and no known exposure to insects or animals. His vital signs were notable for hypertension 167/103 mmHg. On physical exam, he had diffuse non-blanching, well-defined, scaly papules on bilateral lower extremities with localized erythema, excoriations, and mild pitting edema and heavy clustering on lower back and sacrum.

Results: Dermatology was consulted. Lesions on his left lower back and on his left medial foot were biopsied Pathologist's Report: "Sections show compact ortho and parakeratosis a band-like lymphohistiocytic infiltrate obscuring the dermal-epidermal junction associated with necrotic keratinocytes and melanophages. Note: The presence of parakeratosis favors a lichenoid drug reaction (includes a fixed drug reaction) over lichen planus."

Discussion: Many medications have been associated with a lichenoid drug reaction. More common medications include ACE inhibitors, betablockers, nifedipine, methyldopa, diuretics, NSAIDs, phenothiazine derivatives, carbamazepine, and phenytoin. Depending on the medication, the onset can be from several days up to a year.

Conclusion: Drug eruptions are easily missed due to their inconsistent temporal relationship between start of drug and rash onset. Providers should be aware of this diagnosis as skin eruptions are often uncomfortable and can resolve with cessation of the offending medication.

\section{Funky Colchicina: Review of Calls to a Statewide Poison Control System}

Austin Williams ${ }^{1}$, Karisa Wymer ${ }^{1}$, Serena Huntington ${ }^{1,2}$, Rais Vohra ${ }^{1,2}$ ${ }^{1}$ UCSF School of Pharmacy, San Francisco, CA, USA. ${ }^{2}$ California Poison Control System Fresno-Madera Division at Valley Children's Hospital, Madera, CA, USA

Background: Colchicine is a narrow-therapeutic window drug used for a number of rheumatologic conditions, and in overdose can oftentimes be fatal.

Research Question: Which risk factors and/or effects are common to patients with severe colchicine toxicity?

Methods: This study is a retrospective case review of moderate and major outcomes due to colchicine toxicity, as reported to a state-wide poison control system from 1997 to 2017. Cases were identified by free text search for colchicine and as well as the American Association of Poison Control Center's generic code for colchicine $(25,800)$. Cases were included if the patient was seen in the hospital and had moderate or major outcomes, and cases were excluded if they were non-exposure, non-human, and/or no clinical data available. Identified cases were reviewed for demographic, clinical, laboratory, and outcome information, and descriptive statistics were performed.

Results: There were 57 cases of acute and chronic toxicity included in the study, with the following outcomes: 13 deaths (23\%), 7 major effects
(12\%), and 36 moderate effects (46\%). Consistent with the multiple organ dysfunction expected with colchicine toxicity, numerous other complications were noted in the cohort including AKI, anemia, leukopenia, thrombocytopenia, troponin elevation, and gastrointestinal symptoms. Significant differences in rates of hepatotoxicity were noted between cases with deaths $(92 \%)$ and survivors $(30 \%)$. Elevated troponin levels $(>0.03 \mathrm{ng} / \mathrm{ml})$ were reported in nine $(69 \%)$ deaths, in one $(14 \%)$ major effect, and in three $(8 \%)$ moderate effect cases. Thrombocytopenia was reported in ten $(77 \%)$ deaths, in five $(71 \%)$ major effect, and in two $(6 \%)$ moderate effect cases.

Conclusion: Colchicine can cause severe toxicity in acute or chronic exposures and lacks a readily available antidote. This study documents trends in toxicity reported to a large statewide poison control system and demonstrates a potential association between hepatotoxicity, elevated troponins and more severe outcomes.

\section{Jagged Little Pill: Methenamine Hippurate Aspiration}

John Thompson ${ }^{1}$, Annette Lopez ${ }^{1,2}$

${ }^{1}$ Oregon Health \& Science University, Portland, OR, USA. ${ }^{2}$ Oregon Poison Center, Portland, OR, USA

Background: Methenamine hippurate $(\mathrm{MH})$ is a prophylactic urinary tract antiseptic that distributes into body fluids. In acidic urine, it is hydrolyzed into formaldehyde. Hippurate is added to maintain acidity since some urinary pathogens raise urinary $\mathrm{pH}$ to prevent formaldehyde formation. A literature review did not find any instances of aspiration of $\mathrm{MH}$ to guide management.

Hypothesis: Formaldehyde production from hydroxylation results in lung injury.

Methods: Single patient case report after clinical encounter.

Results: An 84-year-old female presented with cough and foul tasting sputum after choking on MH 1 day prior while swallowing it with a large gulp of water. She initially struggled for $15 \mathrm{~min}$ due to a coughing bout, and similar bouts have re-occurred intermittently over the last day. Past medical history was significant for recurrent urinary tract infections, hypothyroidism, and gastroesophageal reflux. Her medications include calcium carbonate $650 \mathrm{mg}$ (three times daily), levothyroxine $50 \mu \mathrm{g}$ (daily), MH (500 mg twice daily), and ranitidine (150 mg twice daily). Vitals on presentation were notable for an elevated blood pressure of 162/ $86 \mathrm{mmHg}$, heart rate of 77 beats per minute, respiratory rate of 18 breaths per minute, temperature of $97.9^{\circ} \mathrm{F}$ and oxygen saturation of $95 \%$. No visualized trauma was present on oral examination and pulmonary exam revealed diffuse wheezing. Nebulized albuterol resulted in improved wheezing. Chest radiograph demonstrated a new left upper lobe mass, inconsistent with her suspected aspiration. Given new wheezing, persistent coughing, and concern for retained pill fragments, pulmonology consultation was sought. Emergent bronchoscopy revealed patchy and incompletely circumferential whitish plaques within the right main stem bronchi as well as proximal bronchus intermedius and endobronchial mucosal injury suggestive of chemical pneumonitis.

Conclusion: This case illustrates injury after $\mathrm{MH}$ aspiration and that emergent bronchoscopy should be considered to prevent further bronchial injury.

\section{7. \#HiddenPoisonNextDoor: Breaking News and its Effects on Poison Center Calls and Social Media Presence}

Adam Blumenberg ${ }^{1,2}$, Fiorella Carhuaz ${ }^{2}$, Sandra Giffin ${ }^{1,2}$, Annette Lopez $^{1,2}$

${ }^{1}$ Oregon Health \& Science University, Portland, OR, USA. ${ }^{2}$ Oregon Poison Control, Portland, OR, USA

Background: On February 3, 2016, an article revealing "alarmingly high arsenic, cadmium levels near schools" was published, prompting community members to seek information regarding these metals' health effects. 
Research Question: Characterize calls placed to the PC, while monitoring effects on PC's social media presence.

Methods: Retrospective chart review of PC calls and Twitter trends for 1 month post event.

Results: There were 117 calls involving 123 individuals. The maximum number of daily calls, 15 , occurred 2 days after the news were released. Health professionals accounted for $63 \%$ of calls, community members were $35 \%$ and $2 \%$ involved community leaders. In $46 \%$ of calls, information was provided. Levels of the heavy metals were managed in $52 \%$ of calls, and $2 \%$ of calls involved arranging presence of PC members at community forums. Ages were obtained for 65 of 123 individuals, and the average age was 13 (range of four months to 59 years). Gender information was obtained on 67 calls, and females accounted for $53.7 \%$. The PC Twitter account gained 30 new followers over the month. $\mathrm{PC}$ received multiple calls regarding this event, with a noted delay of 2 days for the maximum number of calls, likely due to the spread of the news. Most calls were by health care professionals, and the community accounted for one third of calls. Initially, calls were informational; however, as the situation progressed, calls were more likely for interpretation of laboratory results. The average age of the individuals discussed was 13, likely reflecting exposures near schools. Finally, the event coincided with an increase in Twitter account followers, likely reflecting the community's interest in obtaining the PC's latest general information from social media.

Conclusion: After a community-wide exposure, $\mathrm{PC}$ is likely to provide information via both phone calls and social media presence.

\section{Deliberate Thallium Poisoning: a Case Report}

Ming Li Wu ${ }^{1}$, Jou Fang Deng ${ }^{1}$, Nathan Kunzler $^{2}$, Peter Chai ${ }^{2,3}$, Timothy Erickson $^{2,4}$, Edward Boyer ${ }^{2,3}$, Bryan Hayes ${ }^{5}$, Chen-Chang Yang ${ }^{6,7}$

${ }^{1}$ Taipei Veterans General Hospital, Taipei, Taiwan. ${ }^{2}$ Department of Emergency Medicine, Brigham and Women's Hospital, Boston, MA, USA. ${ }^{3}$ The Fenway Institute, Boston, MA, USA. ${ }^{4}$ Harvard Humanitarian Institute, Boston, MA, USA. ${ }^{5}$ Department of Emergency Medicine, Massachusetts General Hospital, Boston, MA, USA. ${ }^{6}$ Institue of Environmental and Occupational Health Sciences, National YangMing University, Taipei, Taiwan. ${ }^{7}$ Division of Clinical Toxicology and Occupational Medicine, Taipei Veterans General Hospital, Taipei, Taiwan

Background: Thallium poisoning is a rare event requiring a high index of clinical suspicion. The presentation is subtle and may be difficult to diagnose. Without prompt recognition of thallitoxicosis, severe neurotoxicity and death may ensue.

Methods: A healthy 31-year-old male presented with gradual, progressive distal neuropathy, dysarthria, and weight loss. He was hospitalized for 3 days with symptomatic treatment. Four weeks later, he developed alopecia, myalgias, and progressive paresthesia of his extremities. Forty days later, he was hospitalized with lower extremity pain, paresthesias, and alopecia. He had painful feet, leg weakness, blurred vision, dysarthria, dysmetria, nystagmus, and poor ocular accommodation with Aldrich-Mees lines on his fingernails. He was admitted with the diagnosis of polyneuropathy, suspected dysimmune neuropathy, or heavy metal poisoning.

Results: Hair, urine, and whole blood specimens demonstrated thallium levels of $10.199 \mathrm{ppm}, 616.79 \mu \mathrm{g} / \mathrm{L}$, and $111.64 \mu \mathrm{g} / \mathrm{L}$, respectively. EMG demonstrated spontaneous discharges in the tibialis anterior muscles consistent with a sensorimotor polyneuropathy and axonal loss. Brain MRI demonstrated abnormal signal of bilateral inferior olivary nuclei. With the diagnosis of thallium poisoning, antidotal therapy with oral Prussian blue $1 \mathrm{~g}$ TID was administered for 3 weeks and $1 \mathrm{~g}$ BID 2 weeks after, resulting in improvement of neuropathic pain. Further investigation revealed the poisoning to be perpetuated by a third party in a presumed homicide.

Discussion: This case illustrates the gradual nature of thallium poisoning with distal axonopathy confirmed by EMG. Suspicion of thallium poisoning should be considered in the context of new painful paresthesias, Mees' lines and alopecia. Diagnosis can be confirmed using urine, whole blood or hair samples.

Conclusion: Thallium poisoning should be considered in patients with neurotoxicity, GI symptoms, and alopecia, particularly in countries with access to thallium. With toxicity, malicious or homicidal intent should be suspected. Prussian blue is indicated once the diagnosis of thallium poisoning is confirmed.

\section{Quantitative Analysis of a Falsely Elevated Serum Creatinine Following a Nitromethane Ingestion}

David Derkits, William Meggs, Jennifer Parker Cote

East Carolina University-Brody School of Medicine, Greenville, NC, USA

Background: Nitromethane is a solvent used in industrial organic synthesis and as a fuel or fuel additive (often with methanol) in motorsports and hobbies. Serum nitromethane causes falsely elevated serum creatinine levels at institutions that use Jaffe-based assays to measure creatinine; enzymatic assays are not affected.

Methods: This is a case report involving a 21-month-old girl who ingested an unmeasured quantity of a fuel containing methanol, nitromethane $(20 \%)$, and lubricants, formulated for use in radio-controlled toys. The patient was initially evaluated for methanol toxicity and was treated empirically. Methanol was below detection by $19 \mathrm{~h}$ post-ingestion, but a serum creatinine of $2.63 \mathrm{mg} / \mathrm{dL}$ (from a Jaffe assay) raised concern for kidney injury, until a repeat serum creatinine using an enzymatic assay came back within normal limits. The discrepancy was attributed to nitromethane. The patient remained inpatient for further evaluation of non-specific lab abnormalities, which permitted trending of her serum creatinine.

Results: During the patient's inpatient stay, a total of seven serum creatinine levels were obtained using the Jaffe assay, along with four levels using the enzymatic assay. While the enzymatic assay levels were all normal-range, the Jaffe assay levels showed a linear decline over time (correlation coefficient -0.99 ).

Conclusion: This case demonstrates a falsely elevated Jaffe assayderived serum creatinine in the context of nitromethane ingestion. The circumstances of this case permitted this relationship to be studied in greater depth. Jaffe-derived creatinine levels, when plotted, showed a convincing linear decline, consistent with zero-order elimination of an interfering substance, likely nitromethane. Using linear regression, we were able to calculate that this patient would require approximately four days for her body to clear the nitromethane. This case demonstrates how Jaffe assay-derived serum creatinine levels might be useful in establishing, quantifying, and trending nitromethane exposures.

130. Effect of Guidelines on FFP/4-Factor PCC Utilization in Treatment of Coagulopathy from Synthetic Cannabinoids Tainted with Long Acting Anticoagulant Rodenticides

Marit Tweet ${ }^{1}$, Michael Wahl ${ }^{2,3}$

${ }^{1}$ Southern Illinois University SOM, Springfield, IL, USA. ${ }^{2}$ Illinois Poison Center, Chicago, IL, USA. ${ }^{3}$ Northshore University HealthSystem, Evanston, IL, USA

Background: In March 2018, cases of coagulopathy consistent with longacting anticoagulant rodenticide (LAAR) poisoning related to synthetic cannabinoid use were reported to a regional poison center (RPC). Upon review of initial cases, it was noted that there was inconsistency in treatment of patients. A consensus guideline was developed with ten medical toxicologists in the Toxikon Consortium. The guideline recommended FFP/4Factor PCC be reserved for use in patients with life-threatening bleeding. Hypothesis: Standardized treatment guidelines from medical toxicologists would reduce use of blood products in the treatment of LAAR coagulopathy. 
Methods: A retrospective chart review of 129 cases reported to a RPC from 3/18/2018 to 6/16/2018 was performed. Forty-eight of the cases came prior to release of the consensus guidelines on April $3^{\text {rd }}, 81$ cases were called on or after April $3^{\text {rd }}$. RPC data was used to record if FFP or 4Factor PCC was used in each case. The data was then divided into Preand Post-guideline groups to calculate the percent of cases who received either FFP, 4-factor PCC, or both. An independent samples $T$ test was performed comparing the usage of FFP/ 4-Factor PCC before and after the guidelines were released.

Results: Twenty-seven of forty-eight cases (55\%) received FFP/4-Factor PCC prior to the release of the guidelines, $33 / 81$ cases $(41 \%)$ after the guidelines were released, received either intervention - at 34\% difference. There was a significant trend for decreased use of FFP/4-Factor PCC after release of the guideline, but it was not statistically significant $(p=0.057$.

Conclusion: The results of this study showed a significant trend towards a change in management with a reduction in usage of FFP/4-Factor PCC in patients with LAAR-related coagulopathy. This highlights the importance and impact of Medical Toxicology expertise on creating guidelines to effectively and efficiently manage treatment of patients during toxic outbreaks.

131. Factor Xa Inhibitor-Associated Hemorrhage is Extremely Rare in an Emergency Department Population

Emily Taub ${ }^{1}$, Patrick Maher ${ }^{2}$, Alex Manini ${ }^{3}$

${ }^{1}$ Division of Medical Toxicology, Ronald O. Perelman Department of Emergency Medicine, NYU School of Medicine, New York, NY, USA. ${ }^{2}$ Department of Emergency Medicine, The Icahn School of Medicine at Mount Sinai, New York, NY, USA. ${ }^{3}$ Division of Medical Toxicology, The Icahn School of Medicine at Mount Sinai, New York, NY, USA

Background: Factor Xa Inhibitors (FXAs), including rivaroxaban and apixaban, are approved for treatment of non-valvular atrial fibrillation and venous thromboembolism. More information is required regarding risks of hemorrhage with their use.

Research Question: To determine the incidence and types of FXArelated hemorrhage in a large ED population.

Methods: This was a retrospective cohort analysis performed at a single tertiary care hospital from 2011 to 2016. Inclusion criteria were all ED patients over 18 years old with either discharge diagnosis of hemorrhage based on ICD-10 codes, or FXA in their medication list. The primary outcome was severe hemorrhage, defined as any of the following: any intracranial hemorrhage (ICH); variceal hemorrhage; ICU admission; initial $\mathrm{SBP}<90 \mathrm{mmHg}$; initial $\mathrm{HR}>125$. Prevalence of FXA-related hemorrhage, incidence of Severe Hemorrhage, and associated clinical factors were analyzed using chi-square and $t$ test, with $5 \%$ alpha.

Results: Over the study period, 608 patients had any hemorrhage; 220 patients were taking FXAs. Patients had mean age 54 years, were $46 \%$ female, and were $17 \%$ Black, $26.7 \%$ White, $3.5 \%$ Asian, $42.1 \%$ other race. No patients died in the ED; 40 patients died during hospitalization (survival to discharge 95\%). Prevalence of FXA-related hemorrhage in 220 patients was $1.4 \%(N=3)$ : 2 on rivaroxaban $(66 \%), 1$ on apixaban $(33 \%, p=\mathrm{NS})$. Ninety-nine percent of patients with any hemorrhage were gastrointestinal: 160 unspecified (26.3\%); 20 diverticular (3.3\%); 85 melanotic $(14.0 \%)$; 1 Mallory-Weiss $(0.16 \%)$; and 4 variceal $(0.66 \%)$. Severe hemorrhage occurred in $41(6.5 \%)$ patients: ICH, 28 (subdural 23, epidural 1, other ICH 4); variceal, 4; ICU admission, 3; $\mathrm{SBP}<90$, 5; and HR > $125,2.0 .5 \%$ of all hemorrhages were FXA-related; all were minor hemorrhages.

Conclusion: The incidence of FXA-associated hemorrhage was extremely low in this large retrospective cohort. FXAs were not significantly associated with severe hemorrhage. Further prospective studies are warranted to confirm these findings.
132. Chemical and Radiation Preparedness Training for Public Health and Nursing Students: an Under-Utilized Disaster Response Demographic

Sukhshant Atti ${ }^{1}$, Aynur Sahin ${ }^{1}$, Emily Kiernan ${ }^{1}$, Mark R Layer ${ }^{1}$, Shaikhah Alotiabi ${ }^{1}$, Waleed Alsukaiti ${ }^{1}$, Ziad Kazzi ${ }^{1,2}$

${ }^{1}$ Emory University School of Medicine, Atlanta, GA, USA. ${ }^{2}$ Georgia Poison Center, Atlanta, GA, USA

Background: Public health (PH) and nursing students are an underutilized demographic in disaster response. Knowledge of the disaster response phase may enhance student understanding of preparedness and provide response capabilities.

Hypothesis: A single 4-h simulation-based training session, with toxicologists as instructors, can effectively improve $\mathrm{PH}$ and nursing student knowledge, and skills, in chemical and radiation response, despite minimal prior experience.

Methods: A convenience sample was used to test $\mathrm{PH}$ and nursing students in a response training program. An introductory lecture and simulation training reviewed: mass casualty care, triage, personal protective equipment, decontamination, and chemical and radiation exposure toxidromes. An examination was administered pre-training, and then post-training, to evaluate relevant training, knowledge, risk perception, and comfort in response capabilities to chemical and radiation incidents. Results: Forty-two students attended the course; $n=39$ were included in the study. Seventy-two percent $(n=28)$ of participants had no prior disaster training. Overall, there were significant differences between the pre-test and post-test scores for all students [95\% CI 5.4 (4.7-6.1); $p<0.0001$, paired $t$ test]; maximum score 15/15. Comparing scores of nursing and PH students, despite statistical difference in pre-test scores (median, IQR 9.0 (7.5-10 \pm 2.0$) ; 7.0$ (5.7-9.0) respectively; $p=0.048$, Mann-Whitney $U$ test), there were no statistical differences in post-test scores (median, IQR 14.0 (13.0-14.0); 13.0 (12.0-14.0), respectively, Mann-Whitney $U$ test). All students recognized nerve agent toxidrome and performed SALT triage after the training $(p<0.0001$, McNemar test). Subjectively, participant comfort level in responding to a chemical or radiological incident improved ( $p<0.0001$, McNemar test). Individual risk perception for chemical or radiological disasters did not improve after training.

Discussion: Improvement in knowledge and comfort was demonstrated, irrespective of previous experience.

Conclusion: Simulation-based training of chemical and radiation disaster preparedness, led by medical toxicologists, is an effective means of educating $\mathrm{PH}$ and nursing students, with minimal prior fluency.

\section{Attitudes Toward and Familiarity with Radiation Emergency Medicine Among US Medical Toxicologists}

Eungjae Kim ${ }^{1}$, Brian Murray $^{2}$, Samuel Ralston ${ }^{2}$, Tim Moran ${ }^{2}$, Brent Morgan $^{2}$, Ziad Kazzi ${ }^{2}$

${ }^{1}$ Emory University, Atlanta, Georgia, USA. ${ }^{2}$ Emory University, Atlanta, Georgia, USA

Background: The threat of a large-scale radiation emergency has increased with advancements in nuclear programs and heightened political tensions. This highlights the need for radiation subject-matter experts who can provide clinical guidance to providers and stakeholders. However, the rarity of radiation emergencies has resulted in a paucity of such experts and a lack of familiarity with the management of radiation injuries among healthcare providers.

Methods: We performed a cross-sectional electronic survey of ACMT's full members to assess (1) their past experiences and comfort level treating radiation injuries, (2) their attitudes toward their role in a radiation emergency, and (3) their knowledge of Radiation Medicine.

Results: The survey was sent to 517 full ACMT members of which 114 $(22.1 \%)$ responded. Half of the respondents had received radiation training during the previous 5 years. A quarter had cared for patients exposed to radiation, and $13 \%$ had cared for patients contaminated with 
radioactive material. Respondents who had previously cared for a radiologically contaminated patient $(\mathrm{OR}=2.2,95 \% \mathrm{CI}=1.39-3.56)$, were willing to respond/participate in a radiologic emergency $(\mathrm{OR}=1.3$, 95\% $\mathrm{CI}=1.06-3.59)$, considered themselves a top-tier expert with the subject matter $(\mathrm{OR}=1.2,95 \% \mathrm{CI}=1.08-1.4)$, or were comfortable assessing radiation poisoned patients $(\mathrm{OR}=1.3,95 \% \mathrm{CI}=1.14-1.51)$ performed significantly better on the knowledge test. Twenty percent of respondents scored greater than or equal to $80 \%$ on the test.

Conclusion: Medical toxicologists are trained and positioned to assist in a catastrophic radiation emergency. We have potentially identified a sizable group of medical toxicologists who self-identify as experts and who have previously cared for radiation patients. We postulate that this group possesses the expertise required to aid in the care of patients poisoned and contaminated by radiation. Moving forward, we need to expand radiation training among medical toxicologists and better define their role in a national or local radiation emergency.

\section{Concentrated Hydrogen Peroxide Ingestions Treated without Hyperbaric Therapy}

Lauren Porter ${ }^{1,2}$, Erik Fisher ${ }^{1,2}$, Anne-Michelle Ruha ${ }^{1,2}$, Daniel Brooks $^{1,2}$, Craig Heise ${ }^{1,2}$, Steven Curry ${ }^{1,2}$

${ }^{1}$ Banner-University Medical Center Phoenix, Phoenix, AZ, USA.

${ }^{2}$ University of Arizona College of Medicine-Phoenix, Phoenix, AZ, USA

Background: Ingestions of concentrated hydrogen peroxide may result in portal venous gas with and without neurological symptoms. Treatment with hyperbaric therapy (HBT) is currently recommended. Few reports exist in the literature of patients managed without HBT. We present three patients with portal venous gas after $\mathrm{H}_{2} \mathrm{O}_{2}$ ingestions that had resolution of symptoms and radiographic findings without HBT.

Cases: A 63-year-old man ingested $50 \% \mathrm{H}_{2} \mathrm{O}_{2}$. He developed vomiting without other symptoms. CT imaging demonstrated emphysematous gastritis and extensive air in the portal venous system and left gastric vein. Upon transfer $5 \mathrm{~h}$ after ingestion, his only symptom was sore throat. $\mathrm{He}$ remained asymptomatic and was discharged home $16 \mathrm{~h}$ after ingestion. No repeat CT imaging was obtained as patient remained asymptomatic. A 54-year-old man drank $35 \% \mathrm{H}_{2} \mathrm{O}_{2}$. He vomited multiple times and was stridulous but without neurological deficits. He was intubated and transferred to our facility. Serial CT imaging demonstrated portal venous gas and pneumomediastinum that resolved on day 4. EGD revealed grade II caustic esophageal injury without perforation. He was extubated and transferred to psychiatry. A 16-year-old girl ingested $35 \% \mathrm{H}_{2} \mathrm{O}_{2}$. She had epigastric discomfort and vomited twice. Imaging showed extensive portal venous gas and mural gas in the gastric fundus. Upon transfer to our facility, $14 \mathrm{~h}$ after ingestion, repeat imaging was performed that showed almost complete resolution of her portal venous gas. She remained asymptomatic for over $24 \mathrm{~h}$ and was transferred to psychiatry. Conclusions: Most published literature recommends HBT for ingestions of $\mathrm{H}_{2} \mathrm{O}_{2}$ whenever there is radiographic evidence of gas bubbles, regardless of the presence of neurological symptoms. Our series demonstrates patients with portal venous gas and without neurological symptoms after $\mathrm{H}_{2} \mathrm{O}_{2}$ ingestion can improve without HBT.

135. Immune Related Adverse Events and Monoclonal Antibody Immunotherapy: a Case of Pembrolizumab-Associated Hypophysitis

Marlena Wosiski-Kuhn, Mary Wittler

Wake Forest School of Medicine, Winston Salem, NC, USA

Background: Cancer immunotherapies are associated with a range of immune related adverse events (IRAEs). We present a rare case of pembrolizumab IRAE of central adrenal insufficiency (hypophysitis).

Hypothesis: Pembrolizumab, a humanized monoclonal antibody recently approved to treat unresectable metastatic cancers, triggered hypophysitis with adrenal insufficiency.
Methods: This case report was generated by single chart review. A 77year-old man with metastatic urothelial cancer on pembrolizumab presented with a 10-day history of overwhelming fatigue, sleepiness, dyspnea on exertion, generalized weakness, nausea, depressed appetite, and mild global headache. Vital signs were temperature $98.3^{\circ} \mathrm{F}\left(36.8^{\circ} \mathrm{C}\right)$, BP 113/85 mmHg, pulse 88 beats/min, RR 16 breaths/min, and oxygen saturation $94 \%$ (room air). Initial evaluation showed sodium $134 \mathrm{mmol} / \mathrm{L}$ and random evening cortisol $0.5 \mu \mathrm{g} / \mathrm{dL}$ (ref. $2.0-10 \mu \mathrm{g} / \mathrm{dL}$ ); otherwise CXR, CT head, CBC, CMP, urinalysis, lactic acid, BNP, CK, and TSH were unremarkable. He was admitted for further evaluation of suspected hypophysitis.

Results: Morning labs showed cortisol $1.1 \mu \mathrm{g} / \mathrm{dL}$ (ref. 5.5-20 $\mu \mathrm{g} / \mathrm{dL}$ ) and $\mathrm{ACTH}<5 \mathrm{pg} / \mathrm{mL}$ (ref. $6-50 \mathrm{pg} / \mathrm{mL}$ ), with correspondingly decreased but responsive $250 \mu \mathrm{g}$ cosyntropin stimulation test (cortisol: 30-min $5.8 \mu \mathrm{g} / \mathrm{dL}, 60-\min 8.5 \mu \mathrm{g} / \mathrm{dL}$ ) indicative of central adrenal insufficiency. Pituitary MRI w/wo contrast was normal. The patient's symptoms improved after temporary discontinuation of pembrolizumab and therapy with hydrocortisone replacement.

Discussion: Reported incidence of pembrolizumab-associated hypophysitis is $0.6 \%$. While etiology is unknown, an autoimmune mechanism is suspected. This case typifies clinical presentation. Pituitary MRI with contrast may be normal or have heterogeneous enhancement. For suspected cases, withhold the offending drug during evaluation of endocrine axes. Continued pembrolizumab therapy is guided by hypophysitis severity.

Conclusion: Pembrolizumab-associated hypophysitis is rare but lifethreatening. Increasing popularity and expansion of immunotherapies will undoubtedly increase IRAEs, requiring clinicians to be both vigilant and appropriately educated.

136. Local Anesthetic Systemic Toxicity After Tumescent Lidocaine Anesthesia for Liposuction

Meghan Spyres, Kent Okawa, Elizabeth Moore

University of Southern California, Los Angeles, CA, USA

Background: Use of tumescent lidocaine anesthesia (TLA) is the practice of injecting a local anesthetic containing fluid into subcutaneous tissues for pain relief during liposuction and other cosmetic surgical procedures. Doses up to $55 \mathrm{mg} / \mathrm{kg}$ lidocaine were historically considered safe; however, recent data suggest lower dosing limits, and serious toxicity and deaths are reported despite these limitations.

Hypothesis: Local anesthetic systemic toxicity (LAST) can occur at standard doses during TLA.

Methods: This is a single patient chart review

Results: A 37-year-old woman with no previous medical history and no medications underwent a breast augmentation and SmartLipo ${ }^{\mathrm{TM}}$ at an outpatient surgical center. She received 1,700 mg lidocaine $(29 \mathrm{mg} / \mathrm{kg})$ in a total of $1400 \mathrm{~mL}$ tumescent fluid (lidocaine, sodium bicarbonate, and epinephrine) distributed to the breasts $(900 \mathrm{mg}$ ) and abdomen/flank $(800 \mathrm{mg}$ ). The patient developed seizure-like activity prior to liposuction initiation. TLA fluid was not aspirated. In transport to the hospital, EMS administered $15 \mathrm{mg}$ midazolam IV without resolution of seizure activity. On arrival to the ED, vital signs were HR $120 \mathrm{bpm}$, BP 157/106 mmHg, $91 \% \mathrm{O}_{2}$ saturation on bag valve mask at 151 . Ongoing tonic-clonic seizure activity was present. She was intubated and placed on a propofol and fentanyl drip with resolution of seizure activity. Lidocaine level resulted at $>24 \mathrm{mg} / \mathrm{L}$. The patient was discharged $24 \mathrm{~h}$ later at her neurologic baseline. Discussion with the surgeon and review of the records revealed no TLA composition irregularities or known intravascular injection.

Conclusion: We report a case of status epilepticus LAST after administration of TLA (29 mg/kg lidocaine) during a liposuction procedure. Toxicity occurred at doses below the recommended limits. No known risk factors for LAST (low BMI, carnitine deficiency, CYP 3A4 interactions and advanced age) were present. 
137: Association Between Timing of Botulinum Antitoxin Administration and Duration of Weakness: Experiences of Botulism Management in Thailand

Sahaphume Srisuma ${ }^{1,2}$, Satariya Trakulsrichai ${ }^{1,3}$, Winai Wananukul ${ }^{1,2}$ ${ }^{1}$ Ramathibodi Poison Center, Mahidol University, Bangkok, Thailand. ${ }^{2}$ Department of Medicine, Ramathibodi Hospital, Mahidol University, Bangkok, Thailand. ${ }^{3}$ Department of Emergency Medicine, Ramathibodi Hospital, Mahidol University, Bangkok, Thailand

Background: Botulism is a worldwide problem. In Thailand, the poison control center also helps in diagnosis and management including coordinating antidote transfer.

Hypothesis: Early botulinum antitoxin administration may relate to shorter duration of weakness from botulism.

Methods: This is a retrospective review of botulism cases reported to Ramathibodi Poison Center during 2010 to 2017. Duration of weakness and mechanical ventilation are described and compared between cases receiving botulinum antitoxin within $72 \mathrm{~h}$ and cases receiving antitoxin later than $72 \mathrm{~h}$

Results: Sixteen botulism were diagnosed including 11 cases with food-borne botulism and 5 cases with iatrogenic botulinum toxin injection. Botulinum toxin was confirmed from food in seven cases (type A 3 cases, and type B 4 cases). Nine cases with food-borne botulism were reported to have initial gastrointestinal symptoms with the median onset of $24 \mathrm{~h}$ (range 2 to $72 \mathrm{~h}$ ). The median onset of weakness in food-borne and iatrogenic botulism was $24 \mathrm{~h}(12 \mathrm{~h}$ to 4 days) and 4 days ( $9 \mathrm{~h}$ to 7 days) respectively. All cases reported bulbar weakness. Five cases reported generalized weakness. Botulinum antitoxin was administered in 13 cases. Median time from weakness onset to antitoxin administration was $72 \mathrm{~h}(6.5 \mathrm{~h}$ to 20 days). Duration of weakness was shorter in cases that received antitoxin within $72 \mathrm{~h}$ (eight cases; median 22 days, range 5-30 days) than in cases that received antitoxin later (five cases, median 55 days, range 18-102 days) ( $p$ value 0.028). Endotracheal intubation and ventilation support were performed in seven cases. There was one death from respiratory failure before receiving antitoxin. Tracheostomy was performed in five cases. Mechanical ventilation duration was shorter in cases that received antitoxin within $72 \mathrm{~h}$ (three cases, 14-16 days) than in cases that received antitoxin later (three cases, 20-52 days) ( $p$ value 0.046).

Conclusion: Early botulinum antitoxin administration relates to shorter duration of weakness from botulism.

138. The Medical Toxicology National Residency Match Program 2014-2019

Anthony Pizon ${ }^{1}$, Paul Wax ${ }^{2,3}$

${ }^{1}$ University of Pittsburgh School of Medicine, Pittsburgh, PA, USA.

${ }^{2}$ University of Texas Southwestern Medical School, Dallas, TX, USA.

${ }^{3}$ American College of Medical Toxicology, Phoenix, AZ, USA

Background: While the Accreditation Council for Graduate Medical Education (ACGME) first approved medical toxicology (MT) fellowship training programs in 2001, a formal National Residency Match Program (NRMP) was only implemented in 2014. For the 2019 appointment year, a comparable NRMP process was initiated for two other emergency medicine (EM) subspecialties: clinical ultrasound $(\mathrm{U} / \mathrm{S})$ and emergency medical services (EMS).

Research Question: To describe the MT NRMP data trend over time, and to compare MT NRMP data to other EM subspecialty data.

Methods: NRMP MT data for appointment years 2014-2019 and NRMP U/S and EMS data for appointment year 2019 were reviewed. Total number of programs, positions, and applicants, and proportion of filled programs and filled positions were analyzed by year and subspecialty.
Results: From 2014 to 2019, the total number of MT programs were 25, $25,26,26,27$, and 28, respectively. During this period, the number of applicants were $24,35,21,42,40$, and 41 . The proportion of filled programs ranged from a low of $48 \%$ in 2014 to a high of $66.7 \%$ in 2018. The proportion of filled positions ranged from $46.3 \%$ in 2016 to $74.5 \%$ in 2017 and 2018. For appointment year 2019, 50\% of programs and $66.7 \%$ of positions filled. For 2019 appointment year, U/S had 116 programs and 194 positions and filled $49.0 \%$ of available positions. EMS had 55 programs and 90 positions and filled $75.6 \%$ of available positions. Conclusion: NRMP participation allows clear benchmarking of fellowships, applicants, and available positions. The applicant pool for MT has remained flat over the last 3 years despite an increase in available training sites and positions. Moreover, other emergency medicine subspecialties will continue to compete for strong fellowship candidates. Further study is needed to determine motivation for residents pursuing MT fellowships.

139. Implementing a Quality Measure Performance Program for Medical Toxicology Patients

Anthony Pizon ${ }^{1}$, Paul $\mathrm{Wax}^{2}$, Sharan Campleman ${ }^{2}$, Dana Karshenas ${ }^{2}$, Jeffrey Brent ${ }^{3}$

${ }^{1}$ University of Pittsburgh School of Medicine, Pittsburgh, PA, USA.

${ }^{2}$ American College of Medical Toxicology, Phoenix, AZ, USA.

${ }^{3}$ University of Colorado School of Medicine, Denver, CO, USA

Background: The Centers for Medicare and Medicaid Services (CMS) has overseen the development of hundreds of quality measures by national medical societies directed at areas with perceived practice performance gaps. Given the absence of quality measures in medical toxicology (MT), the American College of Medical Toxicology (ACMT) developed a program specific for MT patients.

Hypothesis: ACMT can implement a quality performance improvement strategy for MT patients through voluntary reporting to the Toxic Investigators Consortium Registry (ToxIC).

Methods: Over an 18-month period in 2016-2017, ACMT developed nine quality measures that could be used to evaluate the care of a MT patient. In December 2017, CMS approved six of these performance measures on the following topics: opioid misuse screening, pregnancy testing, timely ECG assessment in drug ingestions, appropriate treatment of acetaminophen ingestions, assessment of suspected toxic alcohol exposures, and repeat assessment of salicylate concentrations in overdose patients. Beginning January 1, 2018, data on these performance measures was collected as part of the CMS-approved ToxIC Qualified Clinical Data Registry (QCDR), a new component of the ACMT ToxIC Registry.

Results: Thirty-two medical toxicologists from 11 ToxIC sites participated in the ToxIC QCDR during its first 6 months. Data was obtained on 467 cases that met criteria for opioid screening, 209 cases that met criteria for pregnancy testing, 294 cases that met criteria for EKG assessment, 49 that met criteria for treatment of acetaminophen ingestions, 11 that met criteria for toxic alcohol assessment, and 11 that met criteria for serial salicylate determinations. Performance rates varied from a low of $29.4 \%$ for the acetaminophen measure to a high of $84.6 \%$ for the salicylate measure.

Conclusion: Medical Toxicologists are now able to report on quality measures to evaluate the treatment of MT patients. Initial data collection will allow for valuable benchmarking of current practice.

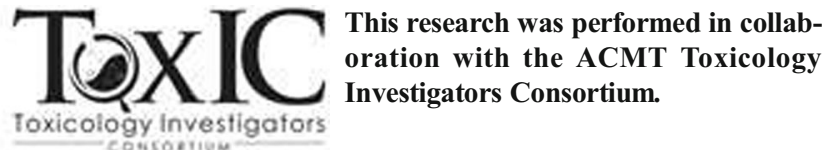




\section{ECG Assessment in Acute Overdoses}

Paul Wax ${ }^{1,2}$, Anthony Pizon $^{3}$, Sharan Campleman ${ }^{1}$, Dana Karshenas ${ }^{1}$, Jeffrey Brent ${ }^{4}$

${ }^{1}$ American College of Medical Toxicology, Phoenix, AZ, USA.

${ }^{2}$ University of Texas Southwestern Medical School, Dallas, TX, USA.

${ }^{3}$ University of Pittsburgh School of Medicine, Pittsburgh, PA, USA.

${ }^{4}$ University of Colorado School of Medicine, Denver, CO, USA

Background: Cardiotoxicity from xenobiotics involved in overdose is a major cause of poisoning morbidity and mortality. Screening and clinical vigilance can identify early warning signs of cardiovascular decompensation.

Research Question: To investigate the proportion of drug overdose patients who receive EKGs within 60 min of arrival to the emergency department (ED).

Methods: Data was collected as part of the Centers for Medicare and Medicaid Services (CMS) approved ToxIC Qualified Clinical Data Registry (QCDR), a component of the ACMT ToxIC Registry. Participants were asked to report on whether an ECG had been obtained as part of the toxicological evaluation. The ECG could have been ordered by any of the patient's health care providers including the triage nurse, EM physician, or medical toxicologist. The denominator was all intentional pharmaceutical overdoses of any age who present to the emergency department, and the numerator was patients who have an ECG within 60 minutes of arrival to the ED. Exclusions include patients who present in cardiac arrest, exploratory pediatric ingestions with non-cardiotoxic drugs, and patients transferred from another hospital.

Results: During the first 6 months of data collection, data was submitted by 29 providers at 9 ToxIC sites. Then, 294 patients met the denominator. Seven of these had incomplete data or denominator exclusions. Further, 130 had an ECG within $60 \mathrm{~min}$. The performance rate who met the metric was $45.9 \%$.

Conclusion: More than $50 \%$ of all intentional pharmaceutical overdoses do not receive an ECG within 60 min after presentation to the ED. These data underscore the importance of medical toxicologists working with their institutions' emergency departments to assure that ECGs are done in a timely fashion on patients known or suspected to have had an intentional pharmaceutical overdose.

\section{ToxIC}

This research was performed in collaboration with the ACMT Toxicology Investigators Consortium.

\section{Pregnancy Testing in Toxicological Evaluations}

Paul Wax ${ }^{1,2}$, Anthony Pizon ${ }^{3}$, Sharan Campleman ${ }^{1}$, Dana Karshenas ${ }^{1}$, Jeffrey Brent ${ }^{4}$

${ }^{1}$ American College of Medical Toxicology, Phoenix, AZ, USA. ${ }^{2}$ University of Texas Southwestern Medical School, Dallas, TX, USA. ${ }^{3}$ University of Pittsburgh School of Medicine, Pittsburgh, PA, USA. ${ }^{4}$ University of Colorado School of Medicine, Denver, CO, USA

Background: Up to $5 \%$ of birth defects are linked to pharmaceuticals, occupational, or environmental exposures. Early identification of maternal fetal risk and management may improve outcomes.

Research Question: To investigate the proportion of women of childbearing age (12-60 years) seen by medical toxicologists who receive a pregnancy test prior to ED discharge or within $24 \mathrm{~h}$ of hospital admission. Methods: Data was collected as part of the Centers for Medicare and Medicaid Services (CMS) approved ToxIC Qualified Clinical Data Registry (QCDR), a component of the ACMT ToxIC Registry. Participants were asked to report on whether a pregnancy test had been obtained as part of the toxicological evaluation. The pregnancy test could have been ordered by any of the patient's health care providers including the EM physician or medical toxicologist. The denominator was the number of women of childbearing age (12-60 years) in the emergency department or inpatient setting with a suspected toxicologic exposure, and numerator was the actual number of patients who received a pregnancy test prior to emergency department discharge or within $24 \mathrm{~h}$ of hospital admission. Exclusions included women who have had a hysterectomy or oophorectomy, minor dermal caustic exposure, and woman who are postmenopausal.

Results: During the first 6 months of data collection, data was submitted from 29 providers at 9 ToxIC sites. During this period, 214 women met the denominator. Five of these had incomplete data and 11 had denominator exclusions. A pregnancy test was documented in 146 cases. The performance rate who met the metric was $73.7 \%$.

Conclusion: More than $25 \%$ of women (12-60 years) do not receive a pregnancy test as part of their toxicological evaluation. These data indicate the importance of medical toxicologists being vigilant to assure that pregnancy testing is done on women who are suspected of having a significant toxic exposure.

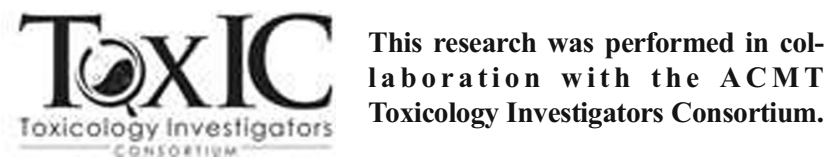

142. The Novel Use of an Emergency Department-Based ICU in the Care of the Poisoned Patient

John Greenwood ${ }^{1,2}$, Jennifer Love ${ }^{1}$, Anita Mudan ${ }^{1}$, Carolyn Stickley ${ }^{1}$, Victoria Zhou ${ }^{1}$, David Jang ${ }^{1}$

${ }^{1}$ Department of Emergency Medicine, Hospital of University of Pennsylvania, Philadelphia, PA, USA. ${ }^{2}$ Department of Anesthesiology and Critical Care Medicine, Philadelphia, PA, USA

Background: The Resuscitation \& Critical Care Unit (ResCCU) is a novel ED-based ICU designed to provide early critical care services to ED patients. One of the roles of the ED-based ICU is to provide more focused care to critically ill patients.

Objective: This study sought to identify predictors of short-stay ICU level care in poisoned patients.

Methods: We conducted a retrospective, single-center case study of poisoned patients $>18$ years old, presenting to an academic, urban hospital ED over 16 months. Patient demographics, relevant drug concentrations, physiologic variables, and severity of illness scores were extracted from electronic medical records and compared between patients who required long and short ICU care (> $24 \mathrm{~h}$ vs. $<24 \mathrm{~h}$ ).

Results: A total of 59 ED visits with a tox-related illness were analyzed. There were 25 female patients and 34 males. Sixteen visits required $>24 \mathrm{~h}$ of ICU care, 13 resulted in short ICU stay and were discharged directly from the ResCCU, 29 required no further ICU time and were sent to the floor, and 1 patient expired. No patients who were admitted to the floor had a step-up to the ICU in either 24 or $72 \mathrm{~h}$. The following toxicology cases were examined: 16 ingestion/overdose, 2 carbon monoxide, 8 alcohol abuse, 13 alcohol withdrawal, 2 heroin abuse, 5 polysubstance abuse, 1 other withdrawal, and 12 other. There were 9 patients that required vasoactive medications and 18 that required mechanical ventilation. APACHE-II scores for patients were as follows: 9 had score $0-10,16$ had a score 11-20, 23 had a score $21-30,1$ had a score $31-40$, and 1 had a score $>40$. Conclusions: Patients who were treated in the ED-based ICU were frequently able to be either discharged home or admitted to a regular floor after their initial stabilization, and none had an ICU step-up. 
143. Estimating the Risk of Long-Term Health Effects Following Acute Exposure to Cholinergic Agents

Kevin Wegman ${ }^{1}$, Laurie Roszell ${ }^{2}$, Jessica Cox $^{3}$, David Bradley ${ }^{3}$, James Madsen ${ }^{4}$, Samuel Ralston ${ }^{5}$, Paul Wax ${ }^{6}$, Charles McKay ${ }^{6}$

${ }^{1}$ Battelle Memorial Institute, Columbus, OH, USA. ${ }^{2}$ Army Public Health Center, Aberdeen Proving Ground, MD, USA. ${ }^{3}$ Department of Homeland Security, Aberdeen Proving Ground, MD, USA. ${ }^{4}$ Medical Research Institute for Chemical Defense, Aberdeen Proving Ground, MD, USA. ${ }^{5}$ Emory University School of Medicine, Atlanta, GA, USA. ${ }^{6}$ American College of Medical Toxicology, Phoenix, AZ, USA

Background: The acute effects of many chemicals are well described with supporting data through traditional toxicology studies. In contrast, long-term health effects resulting from single acute exposures are sparsely supported by epidemiological data with wide ranges of uncertainty.

Hypothesis: A toxidrome-based, subject matter expert (SME)-informed iterative approach can overcome limitations in existing literature, allowing extrapolation of chronic health effects from a single acute exposure to chemical agents.

Methods: Clinical and professional expertise of physicians and toxicologists was leveraged to estimate long-term health effect likelihoods following onetime acute exposure to chemicals within the cholinergic toxidrome. Included in this toxidrome are nerve agents and other chemicals that inhibit the action of cholinesterase. Ten SMEs provided quantitative estimates of the likelihood of long-term mild, moderate or severe health effects given a mild, moderate, severe, or life-threatening acute health effect from exposure to a cholinergic agent. Using the Delphi Method, individual responses were collected, combined input reviewed, and initial responses optionally revised.

Results: For mild, moderate, severe, and life-threatening acute effects, mean estimates of the likelihood of long-term effects ranged from 2.8$12 \%, 9.3-21 \%, 12-25 \%$, and $11-54 \%$, respectively (ranges span the multiple long-term effect severities). The consensus estimates were combined with chemical-specific acute dose-response data for six chemicals to yield mild, moderate, and severe long-term health effect curves. An acute sarin inhalation exposure with a dose equivalent to an ECt50 for severe health effects is estimated to result in $29 \%, 13 \%$, and $5.2 \%$ probability of mild, moderate, and severe long-term effects. Probabilities for other chemicals included in the toxidrome (e.g., tabun and soman) were similar, with differences due to the specific acute dose-response.

Conclusion: The curves resulting from this study provide risk assessors and planners with a method to estimate the long-term health effects following acute chemical exposures until more robust data are available.

\section{Pediatric Exposures Related to Medical Marijuana: a Poison Control Prospective}

Serena Huntington ${ }^{1,2}$, Rais Vohra ${ }^{1,2}$, Kyle Brown ${ }^{2}$, Elmira Zamanpour ${ }^{2}$ ${ }^{1}$ California Poison Control System, Fresno-Madera Division at Valley Children's Hospital, Madera, CA, USA. ${ }^{2}$ UCSF School of Pharmacy at Fresno Medical Education and Research Program, Fresno, CA, USA

Background: Medical use marijuana is currently approved in 32 US states, and with this increased access there is a potential for increased exposure in pediatric patients.

Research Question: How does the availability of medical marijuana impact the type and number of pediatric exposures to cannabis?

Methods: This is an IRB-exempt, retrospective review of pediatric exposures reported to a statewide poison control system from 2010 to 2016 . We investigated poison control case records using the American Association of Poison Control Center's generic code for tetrahydrocannabinol, as well a free text search for THC, marijuana, weed, pot, and edible. Cases were included if the patient was younger than 18 years old and excluded if the record was a non-exposure, patient was non-human, the wrong substance was coded, or had no clinical data. Descriptive statistics were performed.
Results: A total of 1188 cases were identified, and 815 cases were included in the study. The median age of exposure was 14 years old. Ingestion exposures occurred in $63 \%$ of cases, followed by inhalation occurring in $22 \%$ of cases, other routes occurring in $1 \%$, and unknown route occurring in $13 \%$. Formulation of THC was $51 \%$ cases exposed to edibles, $11 \%$ exposed to plant material, $4 \%$ exposed to oils, $2 \%$ other, $0.4 \%$ topical, and $31 \%$ were unknown. Reason for exposure in children younger than 5 years old was accidental $(91 \%)$ versus children aged $11-$ 17 years old was more for abuse (78\%). From 2010 to 2016, exposure rates increased from 70 cases in 2010 to 207 cases in 2016.

Discussion: Over 6 years during medical marijuana legalization, exposures rates continually rose to three times that of the first year. Oral exposure to cannabis edibles was the most common scenario.

Conclusion: The increasing trend in pediatric exposures is expected to continue as more states legalize medical and recreational use marijuana.

\section{Prolonged Sedation After Pediatric Exposure to THC,} Confirmed By Elevated Serum Drug Levels

Anita Ma, Robert Goodnough, Kathy Vo

California Poison Control System (CPCS), San Francisco, CA, USA

Background: Calls to the California Poison Control System related to pediatric exposures have risen with legalization of recreational marijuana. Compared to adults, children may experience profound altered mental status, hypotonia, and coma. There are few documented levels of tetrahydrocannabinol (THC) and its metabolites in pediatric patients, and rare reports of prolonged sedation. We present a case of THC exposure in a child with prolonged sedation and a significantly elevated serum THC level.

Case Report: A 5-year-old boy presented to an emergency department with decreased responsiveness, extensor posturing, and neck stiffness. Seventeen hours prior, he had complained of dizziness to his mother. Physical exam demonstrated a heart rate of 174 beats/minute, otherwise normal vital signs, a Glasgow Coma Scale of 8 , and dilated, minimally reactive pupils. A urine drug screen was positive for THC. Quantitative serum testing showed elevated levels of THC and THC metabolites. Eighteen hours after symptom onset, concentrations of THC, 11-OHTHC, and THC COOH were $35.24 \mathrm{ng} / \mathrm{ml}, 63.08 \mathrm{ng} / \mathrm{ml}$, and $671.39 \mathrm{ng} / \mathrm{ml}$, respectively. At $27 \mathrm{~h}$ after symptom onset, concentrations were $1.42 \mathrm{ng} / \mathrm{ml}, 3.59 \mathrm{ng} / \mathrm{ml}$, and 269.27, respectively. A lumbar puncture, CT brain, MRI brain, and EEG monitoring were normal. He stayed 2 days in the intensive care unit. After $36 \mathrm{~h}$ of hospitalization, he responded to commands, but was still non-verbal. He returned to baseline after $48 \mathrm{~h}$. The source of THC was never determined.

Conclusion: Lethargy, ataxia, and gastrointestinal symptoms commonly occur after pediatric exposures to THC; most cases improve after 2 to $24 \mathrm{~h}$. Symptoms beyond $24 \mathrm{~h}$ occur in the minority of cases. Unfamiliarity with effects of THC in pediatric patients may lead to invasive or unnecessary testing. As exposures increase, it is important to describe the prolonged course of altered mental status that may occur to inform future providers.

\section{A Case of Pediatric AB-FUBINACA Toxicity from a} Commercially Produced CBD Product

Tony Rianprakaisang ${ }^{1,2}$, Adrienne Hughes ${ }^{1,2}$, Lauren Murphy ${ }^{1,2}$, Adam Blumenberg ${ }^{1,2}$, Robert Hendrickson ${ }^{1,2}$

${ }^{1}$ Oregon Health and Science University, Portland, OR, USA. ${ }^{2}$ Oregon Poison Center, Portland, OR, USA

Background: AB-FUBINACA is a synthetic cannabinoid known to cause drowsiness, agitation, delirium, and seizures. Cannabidiol (CBD) is a natural cannabinoid extracted from the cannabis plant that is used as an adjunctive treatment for seizures.

Methods: We present a case of CBD oil adulterated with $\mathrm{AB}$ FUBINACA in a pediatric patient. 
Case Report: An 8-year-old boy with a seizure disorder treated with zonisamide was started on CBD oil by his parents who purchased it over the internet from a well-known out of state commercial supplier. One week prior to presentation, they administered CBD oil daily and reported several days of improved seizure control. The day prior to presentation, the child had increased frequency of seizures and had 14 seizures on the day of presentation. On arrival, the patient had depressed mental status, dilated pupils, and intermittent agitation and delirium. His heart rate was elevated (120 bpm) but he had otherwise normal vital signs. He was drowsy with slowed speech and confusion. He experienced three more seizures in the emergency department treated with midazolam and fosphenytoin. He was admitted to the PICU for further workup. Bloodwork, brain MRI, and lumbar puncture including antiNMDA antibody testing were unremarkable. UDS was positive for benzodiazepines and cannabinoids. Continuous EEG revealed evidence of encephalopathy with no clear seizure activity. The CBD oil was obtained and sent for QTOF-MS analysis revealing the presence of cannabidiol and ABFUBINACA. The patient returned to baseline and was discharged home.

Discussion: We present a case of commercially produced CBD oil adulterated with AB-FUBINACA in a pediatric patient associated with increased seizure activity and delirium.

Conclusion: Clinicians should be aware of possible surreptitious contamination of non-pharmaceutical products that patients may be using.

\section{Lactic Acidosis or Not? A Case of Two Acidotic Siblings}

Mirjana Dimovska ${ }^{1}$, Lydia Baltarowich ${ }^{1,2}$, Cynthia Aaron ${ }^{1,3}$

${ }^{1}$ Michigan Regional Poison Control Center, Detroit, MI, USA. ${ }^{2}$ Henry Ford Hospital, Detroit, MI, USA. ${ }^{3}$ Department of Emergency Medicine Wayne State University School of Medicine, Detroit, MI, USA

Background: Ethylene glycol is metabolized to glycolic acid which is responsible for anion gap metabolic acidosis (AGMA), and oxalic acid, causing nephrotoxicity. Glycolic acid interferes with some lactate assays resulting in a falsely elevated level.

Method: A case of two siblings cared for in a pediatric ICU with toxicology consult.

Results: Two siblings presented simultaneously with tachypnea and lethargy requiring intubation. Patient A was an 18-month-old female and patient B was a 2-year-old male. Initial capillary blood gases done on whole blood (WB) using a Radiometer 800 FLEX.

Patient A capillary WB gas: 6.904/21/149/3.9, lactate 22, AG 27. Patient B capillary WB gas: 6.923/19.6/109/3.8, lactate 27, AG 26. Acidosis was refractory to resuscitation. Differential included toxic alcohols/glycols, cyanide, and metformin toxicity. Fomepizole and sodium bicarbonate infusion were initiated within $1 \mathrm{~h}$ after arrival. Repeat lactates done on serum using an AU 680 Beckmann: at $6 \mathrm{~h}$ post presentation, patient $\mathrm{A}$ had a serum lactate of 2 and a simultaneous WB gas lactate of 13.9. Patient B had a serum lactate of 2.5 with a WB gas lactate of 12.5. Predialysis EG levels were $13 \mathrm{mg} / \mathrm{dL}$ for patient $\mathrm{A}$ and $23 \mathrm{mg} / \mathrm{dL}$ for patient B. Low flux, high efficiency $\mathrm{HD}$ was performed. Both siblings made a complete recovery without residual renal insufficiency. Source of exposure remains unclear.

Discussion: Falsely elevated lactates have been described when using whole blood analyzers to measure lactate in the presence of glycolic acid. To determine the accurate lactic acid, a glycolate insensitive assay should be used. The difference between the lactates, termed "lactate gap," is a clue to the presence of EG.

Conclusion: EG poisoning should be considered in patients who present with AGMA, significant lactic acidosis, and a "lactate gap."

\section{The Role of VA ECMO in Pediatric Flecainide and Diltiazem Overdose}

Mirjana Dimovska $^{1}$, Diana Dean ${ }^{1}$, Cynthia Aaron ${ }^{1,2}$, Andrew King ${ }^{1,2}$

${ }^{1}$ Michigan Regional Poison Control Center, Detroit, Michigan, USA.

${ }^{2}$ Department of Emergency Medicine, Wayne State University School of Medicine, Detroit, Michigan, USA
Background: Flecainide is a class $1 \mathrm{C}$ antiarrhythmic that interferes with sodium and potassium conduction potentially leading to dysrhythmias and death. Diltiazem causes hemodynamic collapse in overdose that can be refractory to medical therapy.

Hypothesis: ECMO is an effective therapy for refractory shock in pediatric cardiotoxic overdoses.

Methods: This is a case report of a single patient presenting to a pediatric specialty care facility after transfer from a community hospital. Patient was cared for in a pediatric intensive care unit with Toxicology consultation.

Results: Twelve-year old female presented to an outside hospital after ingestion of 27 tablets of flecainide and 14 tablets of diltiazem ER the night PTA. Initial VS: BP 88/60s, P 79, and 100\% RA. ECG: QRS $220 \mathrm{~ms}$ and QTc $620 \mathrm{~ms}$. She was intubated, started on high-dose insulin at $1 \mu / \mathrm{kg} / \mathrm{h}$, norepinephrine, bicarbonate infusion, and transferred to a pediatric specialty care facility. Despite escalating vasopressors (norepinephrine, epinephrine, phenylephrine), increase of HDI to $2 \mu / \mathrm{kg} / \mathrm{h}$ with additional bolus dosing of $1 \mu / \mathrm{kg}$, and calcium chloride infusion, patient had refractory shock and was cannulated for VA ECMO shortly after arrival. Laboratory studies: $\mathrm{pH} 7.28$ with $\mathrm{pCO}_{2} 27$, bicarbonate 14 , lactate $11.5 \mathrm{mg} / \mathrm{dL}$. Vasopressors were discontinued within $2 \mathrm{~h}$ and insulin after $12 \mathrm{~h}$ of VA-ECMO initiation. Patient was decannulated on HOD 4, extubated on HOD 7 and transferred to inpatient psychiatry on HOD 15. Discussion: VA-ECMO augments poor cardiac output or can be the sole determinant of forward blood flow. Optimal timing of initiation of VAECMO is case-dependent. Lack of response to aggressive therapy should prompt consideration. VA-ECMO can be titrated in concert with patient's underlying cardiovascular status to maintain optimal end organ perfusion. Conclusion: VA-ECMO should be considered as an option for refractory shock secondary to overdose of cardiotoxic medications in the pediatric population.

\section{Iatrogenic Flecainide Overdose in an Infant Resulting in Pacemaker Failure}

Mirjana Dimovska ${ }^{1}$, Diana Dean ${ }^{1}$, Andrew King ${ }^{1,2}$, Cynthia Aaron ${ }^{1,2}$ ${ }^{1}$ Michigan Regional Poison Control Center, Detroit, Michigan, USA. ${ }^{2}$ Department of Emergency Medicine, Wayne State University, Detroit, Michigan, USA

Background: Flecainide is a class $1 \mathrm{C}$ antiarrhythmic with rate-dependent sodium and potassium channel blocking properties.

Hypothesis: Flecainide toxicity in the infant patient elevates pacing threshold resulting in non-capture and cardiovascular collapse.

Methods: This is a single patient case report of a child cared for in a pediatric specialty care center with toxicology consultation.

Results: Patient was 9 month old with double outlet right ventricle with mitral atresia, pacemaker, and hemi-Fontan (baseline $\mathrm{PaO}_{2} 75-85 \%$ ). Flecainide initiated for pre-excitation. Patient returned to the hospital the day of discharge after a 1-day admission for intolerance of feeds. The child was discharged with flecainide dosage $10 \times$ greater due to a pharmacy labeling error. Mom administered a dose of $3.75 \mathrm{~mL}(80 \mathrm{mg}$ ) instead of the prescribed $0.375 \mathrm{~mL}(7.5 \mathrm{mg})$. After receiving flecainide, he developed difficulty in breathing, coughing, and choking. He appeared cyanotic and less responsive. VS: $\mathrm{HR} 60, \mathrm{RR} 32, \mathrm{SpO}_{2} 72 \% \mathrm{RA}$. ECG demonstrated bradycardia with alternating non-conducted atrial and ventricular impulses. He received $0.075 \mathrm{mg}$ epinephrine and was started on an infusion at $0.1 \mu \mathrm{g} / \mathrm{kg} / \mathrm{h}$. He was intubated due to respiratory failure and decreased responsiveness. Cardiology increased the ventricular lead output resulting in capture and his paced HR increased from 60 to 120 . Vitals improved, epinephrine drip was discontinued. Patient's course was complicated by pleural effusions, airway edema, and difficulty meeting feeding goals. He did have two episodes of recurrent PAT managed with propranolol therapy. He was discharged on HOD 15.

Discussion: Flecainide has a narrow therapeutic index and overdose can be fatal. It has a high affinity for open state or inactivated sodium 
channels. Prior reports demonstrate pacemaker capture failure in adults. Treatment options include increased pacemaker output and vasopressors. Conclusion: In infants with pacemakers, flecainide toxicity results in pacemaker capture failure.

150. Prolonged Hypertension, Decreased Ejection Fraction, and Pulmonary Edema After Clonidine Ingestion

Takuyo Chiba ${ }^{1}$, Michael Chary ${ }^{1}$, Alexander Barbuto ${ }^{1}$, Katherine O'Donnell $^{1,2}$, Michele Burns ${ }^{1,3}$

${ }^{1}$ Harvard Medical Toxicology Program, Boston Children's Hospital , Boston, MA, USA. ${ }^{2}$ Division of General Pediatrics, Department of Medicine, Boston Children's Hospital Boston, Boston, MA, USA. ${ }^{3}$ Division of Emergency Medicine, Department of Medicine, Boston Children's Hospital, Boston, Boston, MA, USA

Background: Clonidine overdoses typically present with somnolence, bradycardia, and hypotension. Initial transient hypertension may be observed, although it is usually brief.

Hypothesis: Massive clonidine overdose in healthy pediatric patients may be associated with prolonged hypertension and clinically significant systolic dysfunction. Clonidine toxicokinetics are different than pharmacokinetics.

Methods: This is a single patient chart review. A 17-year-old female with insomnia presented after the ingestion of 160 tablets $0.1 \mathrm{mg}$ clonidine. EMS administered $0.5 \mathrm{mg}$ IV atropine for bradycardia and $2 \mathrm{mg}$ IV naloxone for somnolence. In the emergency department, she was hypertensive, $164 / 147 \mathrm{mmHg}$, and intubated due to worsening mental status. Chest X-ray demonstrated pulmonary edema. We measured serial serum clonidine concentrations every $12 \mathrm{~h}$ for $60 \mathrm{~h}$.

Results: The patient remained hypertensive for $12 \mathrm{~h}$ (mean arterial pressure $112 \pm 6$, diastolic blood pressure $99 \pm 3.5$; median \pm interquartile range) followed by hypotension requiring dopamine, which was weaned off $48 \mathrm{~h}$ after ingestion. Six hours after ingestion, serum clonidine concentration was $38 \mathrm{ng} / \mathrm{mL}$ (reference range $0.5-2.0 \mathrm{ng} / \mathrm{ml}$ ). The apparent half-life of clonidine was $10.66 \mathrm{~h}$ (reported half-life is $12-16 \mathrm{~h}$ in therapeutic use). Fifteen hours after ingestion, echocardiogram demonstrated an ejection fraction of 0.33 and her serum troponin concentration was $0.25 \mathrm{ng} / \mathrm{mL}$.

Discussion: We describe a massive clonidine overdose notable for prolonged hypertension and systolic dysfunction with associated pulmonary edema and troponin elevation. Pulmonary edema with systolic dysfunction has not been reported after clonidine overdose in healthy pediatric population. Although naloxone could have contributed to both hypertension and pulmonary edema, the time course and the documented cardiac dysfunction support clonidine as the primary driver of these clinical effects.

Conclusion: Massive clonidine overdose may cause prolonged hypertension, myocardial systolic dysfunction, and pulmonary edema. Clonidine kinetics in this massive overdose did not show much difference than clonidine kinetics in therapeutic use.

\section{Cyproheptadine as a Cause of Pediatric Toxicity}

Anthony Scoccimarro, Ryan Marino, Shirley Shao, Alexander Sidlak, Michael Lynch

University of Pittsburgh, Pittsburgh, PA, USA

Background: Cyproheptadine is a first-generation antihistamine touted as an "antidote" for serotonin toxicity due to its serotonin antagonism and is also frequently prescribed in pediatrics for a range of conditions. Limited data exists regarding cyproheptadine overdose in the pediatric population. Hypothesis: Cyproheptadine causes antimuscarinic toxicity.

Methods: This is a two-patient case report. Patient 1 is a 15-year-old girl who presented after intentional overdose of her sister's cyproheptadine initially with sedation followed by agitated delirium, garbled speech, mydriasis, and carphologia. Vital signs were remarkable for tachycardia of $164 \mathrm{bpm}$. Patient 2 is a 17-year-old girl who intentionally ingested her own cyproheptadine and also presented with delirium, garbled speech, mydriasis, and carphologia, with tachycardia of $132 \mathrm{bpm}$.

Results: Patient 1 had undetectable serum acetaminophen, salicylate, and ethanol levels and negative urine enzyme multiplied immunoassay technique (EMIT) testing. Urine gas chromatography/mass spectrometry (GC/MS) testing was positive for cyproheptadine and caffeine. EMIT testing for patient 2 was negative. Patient 2 required $2 \mathrm{mg}$ of lorazepam initially with improvement of agitation and did not require further sedation. Electrocardiography for both patients revealed a sinus tachycardia with normal intervals. Each patient was observed for a total of $48 \mathrm{~h}$ before complete symptomatic resolution.

Discussion: In addition to reported serotonergic antagonism, cyproheptadine is a potent antimuscarinic drug capable of causing a constellation of symptoms including delirium, carphologia, mydriasis, and tachycardia. In both these cases, patients required a total of $48 \mathrm{~h}$ of hospitalization with minimal sedation. Cyproheptadine should be used with caution, if at all, in the undifferentiated toxicologic patient where it may exacerbate a mixed toxidrome, and its use for other conditions should be coupled with understanding of its capability for producing such toxicity.

Conclusion: Cyproheptadine can cause antimuscarinic toxicity and should be used with caution.

\section{Partial-Thickness Skin Burn of a 2-Year-Old Boy's Neck from Contact with an Undissolved Single-Use Laundry Detergent Sac Adherent to Clothing After Laundering}

\section{Mary Kate Abbadessa ${ }^{1}$, Fred Henretig ${ }^{2}$, Kevin Osterhoudt $^{2}$}

${ }^{1}$ The Children's Hospital of Philadelphia, Philadelphia, PA, USA. ${ }^{2}$ The Poison Control Center at the Children's Hospital of Philadelphia, Philadelphia, PA, USA

Background: Childhood exposures to single-use laundry detergent sacs have been associated with eye injuries, oral mucosal injuries, vomiting, airway injuries, and central nervous system depression in both Europe and North America. We report an unusual exposure circumstance, dermal exposure to an undissolved detergent sac adherent to clothing after laundering, that resulted in superficial partial-thickness dermal burn.

Methods: This is a single-subject case report. Parental consent was obtained, and photographs were taken to document the exposure route, injury, and recovery.

Results: A 2-year-old boy changed into laundered pajamas before bedtime and was taken to his preschool in those bedclothes. His caregiver noticed the boy to have neck discomfort and discovered a $>5 \mathrm{~cm}$ diameter area of erythema, with central epidermal breakdown, in his right neck fold. Further inspection found an only partially dissolved laundry detergent sac, including both the film outer layer and some of the colored detergent content, affixed to the inside of the boy's pajama neckline. The detergent sac was removed, the boy's neck was irrigated with clean water, and his clothes were changed. The erythema and pain resolved gradually over the next several days, with central scabbing persisting longer, and full recovery was achieved.

Discussion: Concentrated laundry detergent sacs have been observed to cause eye, intraoral, and pharyngeal burns to children after exploratory exposure with loss of sac integrity. Similar injuries have occurred among adolescents performing intentional stunts. We report a case of a superficial partial-thickness burn to the neck of a 2-year-old boy due to unsuspected contact with a detergent sac that did not completely dissolve during the laundering process.

Conclusion: This case demonstrates that detergent sacs may not completely dissolve and rinse away in the laundering process, and that they may adhere to clothing. Undiscovered, these undissolved laundry detergent sacs pose risk of dermal injury. 
153. All That Glitters Is Not Gold: Inhalational Elemental Mercury Toxicity From a Household Exposure

Sukhshant K Atti ${ }^{1}$, Elizabeth Silver ${ }^{2}$, Emily Kiernan ${ }^{1}$, Ziad Kazzi ${ }^{1,2}$, Robert Geller ${ }^{1,2}$, Mark R Layer ${ }^{1}$

${ }^{1}$ Emory University School of Medicine, Atlanta, Georgia, USA. ${ }^{2}$ Georgia

Poison Center, Atlanta, GA, USA

Background: Clinically significant elemental mercury toxicity can resemble infectious illness. Severe morbidity and mortality can be prevented if mercury toxicity is considered early, through a detailed history, and through maintenance of a broad differential diagnosis. We present a case series of patients with inhalational elemental mercury toxicity, which was initially mistaken as streptococcal pharyngitis and viral syndrome.

Methods: Case series of four patients with elemental mercury toxicity confirmed by whole blood assay. Case 1: a 15-year-old female presented to the ED on three occasions with fever, sore throat, maculopapular rash, and cough. On her third visit, she had leukocytosis, thrombocytopenia, tremor, inability to walk, sensory deficits, and pneumonitis on chest radiograph. Case 2: a 13-year-old female presented on three separate ED visits with fever, maculopapular rash, headaches, and sore throat. Case 3: an 11-year-old male presented on three separate ED visits with fever, chills, maculopapular rash, sore throat, and cough. Case 4: a 34-yearold female presented on two separate ED visits with fever, maculopapular rash, sore throat, shortness of breath, and cough. On her second ED visit, she was tachypneic and febrile, in addition to having a severe headache and perioral numbness.

Results: These patients were exposed to elemental mercury that was spilled in their home by case 1 and vacuumed by cases 1 and 4 . They all required chelation with succimer, plus BAL in case 4 . They recovered over the following 2 weeks. Initial whole blood mercury levels were (normal $<20 \mu \mathrm{g} / \mathrm{L}$ ): $>200 \mu \mathrm{g} / \mathrm{L}, 154 \mu \mathrm{g} / \mathrm{L}, 130 \mu \mathrm{g} / \mathrm{L}$, and $171 \mu \mathrm{g} / \mathrm{L}$ on cases 1 to 4 respectively.

Conclusion: Mercury toxicity can mimic a febrile illness. Healthcare providers should have a high index of suspicion for mercury toxicity and other heavy metal toxicities. Though these are rare, the consequences of a misdiagnosis are detrimental.

154. Per- and Polyfluoroalkyl Substances (PFAS) Exposures: an Emerging Concern

D Karshenas ${ }^{1}$, L Nguyen ${ }^{1}$, C Dickenson ${ }^{1}$, C Baum $^{1,2}$, C McKay ${ }^{1,3}, \mathrm{P}$ Wax ${ }^{1,4}$

${ }^{1}$ American College of Medical Toxicology, Phoenix, AZ, USA. ${ }^{2}$ Yale University School of Medicine, New Haven, CT. ${ }^{3}$ University of Connecticut School of Medicine, Farmington, CT, USA. ${ }^{4}$ UT Southwestern Medical School, Dallas, TX, USA

Background: In 1998, the Pediatric Environmental Health Specialty Unit (PEHSU) program was established, which provides consults on toxic exposures, particularly in children and (recently) in pregnant women. Recently, an increasing number of callers to the PEHSUs have called for information about per- and polyfluoroalkyl substances (PFAS).

Research Question: Describe PEHSU consults about PFAS exposures (and possible exposures).

Methods: PEHSU specialists maintain consult records in a national Performance Tracking System. We analyzed PEHSU consults initiated between January 2016 and October 2018 involving PFAS as an agent of concern.

Results: Between January 2016 and October 2018, the PEHSU network provided 90 individual and community level consultations on PFAS: 53 consults in 2016, 21 consults in 2017, and 16 consults from Jan to Oct 2018. Perfluorocarboxylic acids (PFOA), a type of PFAS, was specifically identified as a concern in $65(72.2 \%)$ of these cases. Of these consults, $36(40.0 \%)$ were delivered to health professionals, and $54(60 \%)$ to the public. Of the $56(62.2 \%)$ consults focused on individual families, a potential exposure was reported in 29 cases $(51.8 \%)$, with the most frequent exposure setting being the home (13 cases; $44.8 \%$ ). Together, these families included 18 potentially exposed adult women (including 2 known to be pregnant) and 35 potentially exposed children (14 female, 5 male, 16 gender unknown). The most frequently suspected route of exposure was oral and water. Six of these children reported gastrointestinal symptoms which were thought to be possibly related to PFAS exposure.

Conclusion: Consultations regarding PFAS are not infrequently reported to PEHSUs. Understanding the environmental health risks of these groundwater contaminants is critically important. Further dissemination of PEHSU PFAS educational resources for health care providers and the public should be encouraged.

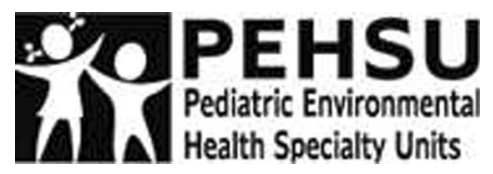

This abstract was supported by the cooperative agreement award number 5 U61TS000238-05 from the Agency for Toxic Substances and Disease Registry (ATSDR). Its contents are the responsibility of the authors and do not necessarily represent the official views of the Agency for Toxic Substances and Disease Registry (ATSDR).

Acknowledgement: The U.S. Environmental Protection Agency (EPA) supports the PEHSU by providing partial funding to ATSDR under InterAgency Agreement number DW-75-95877701. Neither EPA nor ATSDR endorse the purchase of any commercial products or services mentioned in PEHSU publications

155. Development of a Clinical Tool to Assist Providers and Their Pregnant Patients Regarding Environmental Chemical Exposure Concerns

Carrie Dickenson ${ }^{1}$, Dana Karshenas ${ }^{1}$, Elizabeth Nguyen $^{1}$, Carl Baum ${ }^{2,1}$, Paul Wax ${ }^{1}$, Charles McKay ${ }^{1}$

${ }^{1}$ American College of Medical Toxicology, Phoenix, AZ, USA. ${ }^{2}$ Yale School of Medicine, New Haven, CT, USA

Background: Reducing exposure to toxic environmental agents is an area of intervention for reproductive health professionals. In October 2013, the American College of Obstetricians and Gynecologists (ACOG) released a call to action to increase clinician education to reduce the incidence of toxic environmental exposure among women of reproductive age.

Hypothesis: An interactive tool completed by patients to assess exposure and exposure concerns, and used by clinicians to provide risk reduction counseling regarding environmental exposures can be developed.

Methods: As part of the Pediatric Environmental Health Specialty Unit (PEHSU) cooperative agreement with the American College of Medical Toxicology (ACMT), ACMT was tasked with developing a response to the ACOG call to action. In 2016, ACMT convened an expert advisory group comprised of environmental health experts from reproductive health organizations. This group identified topic areas for assessment. Technical writers developed literature reviews and identified consumer resources.

Results: A web-based tool known as the Prenatal Assessment of Environmental Risk (PAER) was developed. It provides an interface for the patients to complete an exposure assessment and a separate interface to guide the clinician's risk reduction counseling. Ten topic areas: Carbon Monoxide, Pesticide Residues, Mercury, Lead, Environmental Tobacco Smoke, Chemical Flame Retardants, Radon, Organic Solvents, Glyphosate, Cosmetics, and Perfumes, are covered. Providers supply their patients with a unique link, which surveys the pregnant patient about potential sources of exposure at the workplace, home, and through consumer purchasing behavior. Results outline potential areas of concern along with points for clinical follow-up. Accompanying continuing 
education modules provide the clinician with examples of low, medium, and higher intensity exposure situations.

Conclusion: Responding to the 2013 call from ACOG for clinical education on reproductive environmental health concerns, ACMT developed the PAER tool for clinicians to use with their pregnant patients. Rollout of this tool is planned for 2019 .

\section{ToP PeHSU}

This abstract was supported by the cooperative agreement award number 5 U61TS000238-05 from the A gen cy for Toxic Substances and Disease Registry (ATSDR). Its contents are the responsibility of the authors and do not necessarily represent the official views of the Agency for Toxic Substances and Disease Registry (ATSDR).

Acknowledgement: The U.S. Environmental Protection Agency (EPA) supports the PEHSU by providing partial funding to ATSDR under InterAgency Agreement number DW-75-95877701. Neither EPA nor ATSDR endorse the purchase of any commercial products or services mentioned in PEHSU publications

156. Impact of Environmental Health Online Education on Clinical Practice

Elizabeth Nguyen $^{1}$, Carrie Dickenson ${ }^{1}$, Dana Karshenas ${ }^{1}$, Carl Baum ${ }^{2,1}$, Charles McKay ${ }^{1}$, Paul Wax ${ }^{1}$

${ }^{1}$ American College of Medical Toxicology, Phoenix, AZ, USA. ${ }^{2}$ Yale School of Medicine, New Haven, CT, USA

Background: Healthcare providers receive relatively little formal education on environmental health during their training. The Pediatric Environmental Health Specialty Unit (PEHSU) Program offers online learning to healthcare professionals through several different modalities, including eLearning modules, and on-demand webinars.

Research Question: Can the PEHSU National Classroom eLearning modules increase the health care providers' knowledge and skills in environmental exposure assessment and risk reduction counseling?

Methods: Six months after a learner completes an eLearning module in the PEHSU National Classroom, the learner is emailed a link to a 15question evaluation survey in SurveyMonkey assessing the impact of the knowledge gained from the eLearning modules on their clinical practice. Using a Likert scale, we assessed clinician's incorporation of environmental factor assessment questions and environmental health risk reduction counseling into their practice. Responses across all nine modules were combined and analyzed to understand the impact of the eLearning modules (regardless of topic) on clinical practice.

Results: Six-month follow up surveys of eLearning module users was conducted from May 2017 to October 2018. Eight hundred seventeen evaluations were sent. Of the 162 responses, $69 \%$ worked in a clinical setting. Seventy-eight percent $(53 / 68)$ of respondents indicated they "Strongly Agree" or "Agree" in response to the question, "After completing these modules, I have incorporated questions about potentially relevant environmental factors in the assessment of a sick child." Seventy-three percent (47/64) of respondents indicated they "Strongly Agree" or "Agree" in response to the question, "After completing these modules, I have added environmental health risk reduction in counseling".

Conclusion: PEHSU eLearning module users have heightened awareness at 6-month follow-up regarding exposure and risk reduction counseling.

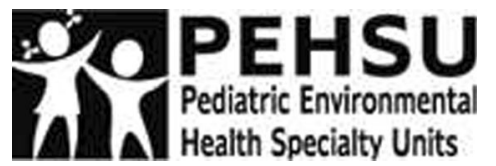

This abstract was supported by the cooperative agreement award number 5 U61TS000238-05 from the
Agency for Toxic Substances and Disease Registry (ATSDR). Its contents are the responsibility of the authors and do not necessarily represent the official views of the Agency for Toxic Substances and Disease Registry (ATSDR).

Acknowledgement: The U.S. Environmental Protection Agency (EPA) supports the PEHSU by providing partial funding to ATSDR under InterAgency Agreement number DW-75-95877701. Neither EPA nor ATSDR endorse the purchase of any commercial products or services mentioned in PEHSU publications

157. A Systematic Review of the Potential Respiratory Sequelae of Exposure to Open Air Burn Pits in Afghanistan and Iraq

Aakash Shah

Rutgers - Robert Wood Johnson Medical School, New Brunswick, NJ, USA

Background: Multiple military sites in Afghanistan and Iraq disposed of waste - often containing a mix of chemicals, paints, medical and human waste, metal and aluminum cans, munitions and other unexploded material, petroleum and lubricant products, plastics, rubber, and wood-in open air burn pits. The resulting exposure to the products of combustion was found to include known toxins such as dioxins. The respiratory sequelae of exposure to open air burn pits among military service members in these settings, however, is not yet clear.

Research Question: The aim of this study was to understand and summarize the current body of evidence on the respiratory sequelae of exposure to open air burn pits.

Methods: This is a systematic review of all peer-reviewed publications indexed in MEDLINE using a clearly defined search strategy and supplemented by relevant citations from government testimony on the respiratory sequelae of exposure to open air burn pits. The results were screened for duplicates, lack of relevance, and non-English publications. Thirty-five studies selected for inclusion and reviewed.

Results: The review revealed that existing evidence for chronic lung disease associated with exposure to open air burn pits varied by condition. The strongest evidence of association was with obstructive lung diseases, including chronic bronchitis, chronic obstructive pulmonary disease, and emphysema. Preliminary evidence of association was also found for asthma, constrictive bronchiolitis, granulomatous disease, and pneumonia.

Conclusion: A systematic review of all peer-reviewed publications indexed in MEDLINE and cited in recent government testimony on the respiratory sequelae of exposure to open air burn pits suggest a link between several respiratory illnesses - most notably chronic bronchitis, chronic obstructive pulmonary disease, and emphysema - and exposure.

158. Case Report of Reversible Cardiomyopathy After Freon Exposure

Amy Fuller, William Meggs, Jennifer Parker Cote

East Carolina University-Brody School of Medicine, Greenville, NC, USA

Background: Freon is a fluorinated hydrocarbon, which is commonly used as coolants or spray propellants. Reports of hydrocarbon inhalation causing cardiotoxic effects commonly describe dysrhythmias as the presenting feature, but there are many potential cardiac effects not yet well described in the literature.

Methods: This is a single patient case report. A 47-year-old male with no known prior cardiac history had two sequential inhalation and ingestion exposures to freon. He presented approximately $7 \mathrm{~h}$ after incident, complaining of progressive dyspnea, chest tightness, and vomiting requiring intubation.

Results: Initial chest X-ray showed bilateral pulmonary opacities and he became acutely hypertensive to $260 / 127 \mathrm{mmHg}$ with pulse rate 160 that 
required multiple antihypertensive medications. Four hours after arrival, troponin was $0.13 \mathrm{ng} / \mathrm{mL}$ and BNP was $24 \mathrm{pg} / \mathrm{mL}$. During the first $24 \mathrm{~h}$ in the ICU, he required $\mathrm{FiO}_{2} 90 \%$, PEEP $20 \mathrm{cmH}_{2} \mathrm{O}$, and paralysis to maintain appropriate oxygenation and ventilation. Echocardiogram showed normal atria, ventricles, and valves, and ejection fraction (EF)

Discussion: While this case report demonstrates freon toxicity-induced cardiomyopathy with reduced EF that resolved in 1 week, it is unclear if the etiology of the heart failure was cardiogenic, non-cardiogenic or a combination.

Conclusion: Toxic inhalation of freon can cause reversible non-ischemic cardiomyopathy.

\section{Occupational Mercury Vapor Toxicity: a Really Painful Diagnosis}

\section{Kelly Johnson-Arbor}

National Capital Poison Center, Washington, DC, USA. MedStar Georgetown University Hospital, Washington, DC, USA

Background: Mercury vapor intoxication can cause severe but nonspecific signs and symptoms in affected individuals. This is a case of occupational mercury vapor toxicity which was initially misdiagnosed as fibromyalgia.

Case report: A 40-year-old female, otherwise healthy and employed as a home health aide, developed severe and constant pains in her extremities, teeth, and neck. She was evaluated by multiple physicians; aside from new-onset hypertension, her physical examination, laboratory testing, and imaging were unremarkable. She was diagnosed with fibromyalgia and prescribed tramadol. At the patient's request, heavy metal testing was ordered; a 24-h urine mercury assay was elevated at $53 \mu \mathrm{g} / \mathrm{L}$, and a whole blood mercury concentration was $34 \mu \mathrm{g} / \mathrm{L}$. Assessment of the patient's home revealed elevated mercury vapor levels in the patient's dresser drawer where she kept her work clothes; workplace testing identified elevated mercury vapor levels outside of the patient's former work location. The work clothes were removed from the home and the patient was treated with succimer chelation therapy, with subsequent decline in both urine and blood mercury concentrations. The patient's pain and hypertension gradually resolved over the next several months, and she was able to discontinue all of her medications.

Discussion: The half-life of elemental mercury is variable and may range from 30 to 90 days. Mercury elimination in this patient was initially rapid (half-life of approximately 2 months) with a slower decline noted months after her diagnosis was established. Whole blood mercury levels are often reported to be elevated only in the short-term period after mercury vapor exposure; in this patient, whole blood mercury was detectable for nearly a year after her reported exposure.

Conclusion: Heavy metal poisoning should be included in the differential diagnosis of patients who present with vague or nonspecific signs or symptoms.

160. Occupational Dermal Exposure to Alpha-Amanitin: Alarm, and Disparity, in Safety Data Sheets

Maricel Dela Cruz ${ }^{1}$, David Vearrier ${ }^{1}$, Amy Behrman ${ }^{2}$, Maryann Hofman $^{3}$, Kevin Osterhoudt ${ }^{3}$

${ }^{1}$ Department of Emergency Medicine, Drexel University College of Medicine, Philadelphia, PA, USA. ${ }^{2}$ Department of Emergency Medicine, Hospital of the University of Pennsylvania, Philadelphia, PA, USA. ${ }^{3}$ The Poison Control Center at the Children's Hospital of Philadelphia, Philadelphia, PA, USA

Background: A worker suffered dermal exposure to a solution of purified alpha-amanitin in the laboratory setting. Reference to the manufacturer's Safety Data Sheet (SDS) alarmed caregivers as it stated, "fatal in contact with skin," whereas another SDS sheet for the same substance labeled it innocuous.
Hypothesis: There are disparities in the information provided by different manufacturer's SDSs for purified alpha-amanitin.

Methods: This is a case report of a healthy 29 -year-old male laboratory worker who presented to an occupational health clinic $30 \mathrm{~min}$ after dermal exposure to alpha-amanitin. Review of the SDS attributed "category 2 " acute toxicity to the skin, and prominently labeled the substance as "fatal in contact with skin." This led to an analysis of other SDSs regarding the hazards of dermal exposure to this form of alpha-amanitin.

Results: Four out of seven SDSs from major manufacturing companies listed alpha-amanitin as "fatal in contact with skin," while one countered that it was benign with dermal contact. No data were found to support the statement that alpha-amanitin was fatal in humans through skin. Our patient remained asymptomatic and on follow-up $48 \mathrm{~h}$ later did not develop any signs or symptoms of toxicity.

Discussion: Alpha-amanitin is a toxic cyclic peptic produced by Amanitin phalloides and can be fatal if ingested. According to the United States Occupational Safety and Health Administration, a skin category 2 designation means that the dermal LD50 of the material is greater than $50 \mathrm{mg} / \mathrm{kg}$ body weight, but less than or equal to $200 \mathrm{mg} / \mathrm{kg}$. Research investigating dermal absorption of alpha-amanitin in mice did not demonstrate the toxin to be absorbed through skin, nor that cutaneously applied alpha-amanitin might have systemic effects.

Conclusion: Inaccurate information in SDSs can be confusing and cause alarm; systems to improve accuracy and consistency of SDSs should be explored.

\section{A Case of Wernicke Encephalopathy Associated with Hyperemesis Gravida}

William Meggs, Samuel Lee, Jennifer Parker Cote East Carolina University, Greenville, NC, USA

Background: Toxicologists are frequently consulted on cases of acute encephalopathy and must consider a differential diagnosis.

Hypothesis: Wernicke encephalopathy secondary to thiamine deficiency should be considered in the differential diagnosis of acute encephalopathy in pregnant women

Methods: This is a case report.

Results: A 27-year-old woman with no prior pregnancies presented to the hospital 17 weeks pregnant with nausea, emesis, and abdominal pain. Right upper quadrant tenderness on examination led to a RUQ ultrasound that found gallbladder sludge. Surgical consultant offered acute cholecystectomy versus expectant management. She chose the latter and improved with IV hydration, ondansetron for nausea. She was discharged on hospital day 3 with a diagnosis of hyperemesis gravida and gallbladder sludge. Three days later, she presented with continued emesis and altered mental status. She and family members denied alcohol or illicit drug use. She reported using bath salts prescribed by a doctor in her bath water. Vital signs were pulse $99 / \mathrm{min}$, blood pressure $115 / 70$, temperature $36.4{ }^{\circ} \mathrm{C}$, respiratory rate 18 , and oxygen saturation $99 \%$. Neurological examination was delirium with waxing and waning mentation. She was intermittently unable to follow commands. Cranial nerve examination was with obvious internuclear ophthalmoplegia with nystagmus in the abducting eyes bilaterally. CT scan of brain was negative. MRI found abnormal T2-weighted signal in the central pons and medial thalami. Radiographic differential included central pontine myelinolysis, demyelinating conditions from malnutrition, Wernicke encephalopathy, and toxic encephalopathy from alcohol use or other drugs. Potassium and albumin were low at $2.7 \mathrm{mEq} / \mathrm{L}$ and $3.2 \mathrm{~g} / \mathrm{dL}$. Thiamine level was below the limits of detection. Alcohol and urine drug screen were negative. Diagnosis was thiamine deficiency secondary to hyperemesis gravida with Wernicke encephalopathy.

Conclusion: Toxicologists are consulted on cases of encephalopathy. In cases of pregnant women with hyperemesis gravida, Wernicke encephalopathy secondary to thiamine deficiency should be considered. 


\section{Tak: The Computational Toxicological Machine}

Michael Chary ${ }^{1}$, Edward Boyer ${ }^{1,2}$, Michele Burns ${ }^{1}$

${ }^{1}$ Boston Children's Hospital, Boston, MA, USA. ${ }^{2}$ Brigham and Women's Hospital, Boston, MA, USA

Introduction: The volume of toxicological knowledge and research has outstripped the ability of the individual clinician to entirely master and stay current with it.

Methods: Tak is a network of first-order logic statements (axioms), derived by committee consensus, weighted by probabilities to capture essential physical exam findings of the four classic toxidromes (sympathomimetic, anticholinergic, cholinergic, sedative-hypnotic), serotonin syndrome, and opioid intoxication. Each axiom relates clinical findings (e.g., tachycardia, bradypnea) to the chance of a toxidrome being present. We trained Tak on 1000 synthetic poisoned patients and evaluated Tak on another independent group of 120 synthetic poisoned patients, which two blinded toxicologists also rated. Each synthetic poisoned patient was generated by choosing three findings from the intended toxidrome and 0-2 symptoms from a "distracting" toxidrome.

Results: Tak correctly identified the toxidrome in $62 \%(628 / 1000)$ of cases in the training data set, most commonly confusing anticholin- ergic with sympathomimetic presentations and opioid with sedativehypnotic presentations. Tak correctly identified the toxidrome in $13 \%$ $(16 / 120)$ of the cases in the testing data set, as compared to $16 \%(19 /$ 120) for toxicologists (inter-rater reliability between raters; 0.81 ). This difference between Tak and the human raters was not statistically significant. Toxicologists and Tak agreed with each other on $39 \%(45 / 120)$ of the cases. The most common sources of disagreement were over anticholinergic vs sympathomimetic toxidromes.

Conclusion: Our study is the first demonstration that toxicological acumen can be digitized. This digitization performs as well as clinicians in identifying at a single point in time toxidromes represented by physical exam abnormalities. Limitations include low accuracy rate for Tak and human raters, which may reflect unrealistic synthetic poisoned patients. We did not consider other features, such as the history, or laboratory findings. Our analysis only considered the most likely toxidrome, but Tak can generate a ranked list of toxidromes.

Author-Abstract Index

Publisher's Note Springer Nature remains neutral with regard to jurisdictional claims in published maps and institutional affiliations. 
Author name Abstract number

Aaron, Cynthia 099, 147, 148, 149

Abbadessa, Mary Kate 152

Abesamis, Michael 043, 063, 064, 110

Abo, Benjamin 099

Abrishamian, Matthew 092

Ackerman, Laurie 016

Aks, Steven E 002, 009, 015

Al-Abed, Yousef 056

Albertson, Timothy 091

Alfaifi, Musa S 022, 046

Alotiabi, Shaikhah 031, 132

Alsukaiti, Waleed 022, 025, 039, 046, 132

Alwasiyah, Dalia 116

Ambros, Victor 055

Armenian, Patil 093

Arnold, Ryan 086

Atti, Sukhshant K 033, 053, 058, 072, 117, 132, 153

Attridge, Rebecca 028

Avera, Robert 040

Baltarowich, Lydia 147

Barada, K 046

Barbuto, Alexander 106, 150

Barton, David 103

Baum, Carl 154, 155, 156

Beauchamp, Gillian 029

Behrman, Amy 160

Bellis, Mitchell 079

Beuhler, Michael 100, 107, 116

Bhalla, Ashish 054

Bilello, Kathryn 093

Black, Elizabeth 076

Bloom, Joshua 056

Blumenberg, Adam 008, 011, 026, 047, 068, 069, 070, 077, 078, 111, 127, 146

Bogucki, Sandy 057

Bonney, Caitlin 083

Boyer, Edward 074, 128, 162

Boyle, Katherine 088

Bradley, David 143

Brent, Jeffrey 071, 112, 118, 139, 140, 141

Brewer, Matthew 097

Briggs, Meredith 024

Brooks, Daniel 134

Brown, Kyle 144

Bryan, Gisela 015

Bryant, David 097

Bunch, Dustin 057

Burkhart, Keith 071

Burns, Michele 106, 150, 162

Burns, Rebekah 041

Calello, Diane 038, 071, 080, 108

Camarena-Michel, Alexa 034

Campleman, Sharan 071, 112, 114, 118, 139, 140, 141

Canning, Joshua 037, 096

Cannon, Robert 029

Cantrell, F Lee 023, 032

Cao, Dazhe 081

Carhuaz, Fiorella 127
Carpenter, Joseph 003, 022, 025, 058, 061, 072, 117

Carreiro, Stephanie 055, 114

Carter Powell, Ashley 037, 121

Chai, Peter 074, 128

Chapman, Brittany 055

Charek, Matthew 104

Chary, Michael 106, 150, 162

Chen, Alex 121

Chhabra, Neeraj 002, 009, 015

Chiba, Takuyo 088, 150

Claudius, Ilene 084

Cook, Matthew 029

Cooke, Lauren 079

Cox, Jessica 143

Curry, Steven 059, 121, 134

Dabrowski, Paul 084

Davidson, Christian 081

Dayton, Christopher 028

Dean, Diana 099, 148, 149

Dela Cruz, Maricel 073, 086, 092, 160

Demestihas, Menelaos 082

Deng, Jou Fang 128

Derkits, David 129

Desiato, Vince 017

Deutsch, Amanda 014

Dhibar, Debaprasad 054

Dickenson, Carrie 154, 155, 156

Dimovska, Mirjana 099, 147, 148, 149

Dolcourt, Bram 099

Dorey, Alyrene 091

Dribben, William 102

Dubin, Jeffrey 007

Dulaney, Anna 107

Dunkley, Camille 022, 025, 039, 117

Durant, Thomas 057

Eggleston, William 095

Ekins, Sean 087

El Zahran, Tharwat 031

Ellis, Judson 109

Erickson, Timothy 074, 128

Ershad, Muhammed 073, 086, 092

Everett, Christopher 028

Fagan, Kathleen 115

Fedoruk, Lynn 024

Fisher, Erik 134

Ford, Jonathan 044, 124

Fox, Erin 014

Fries, Danielle 115

Frohna, William 007

Fuller, Amy 158

Gajdosik, David 014

Ganetsky, Michael 088

Gao, Han 048

Garbosky, Lyudmila 014

Garlich, Fiona 105

Geib, Ann-Jeannette 100

Geller, Robert 117, 153

Giannoutsos, Spiros 051, 087 
Giffin, Sandra 127

Gittinger, Melissa 033, 039, 046, 053

Goodnough, Robert 120, 145

Goshen, Jasmin 001

Graves, Rachel 010, 018, 019

Greenwood, John 142

Gullapalli, Sneha 093

Gutierrez, Gloria 028

Halmo, Laurie 083

Hamilton, Richard 086

Hammack, Jean 066

Hansen, Megan 060, 065

Harmon, Elizabeth 042

Harrison, Robert 115

Hatten, Benjamin 034, 083

Haut, Lindsey 016

Hayes, Bryan 128

Haynes, Ashley 006, 098

He, Mingzhu 056

Hedge, Matthew 099

Heise, Craig 059, 134

Hendrickson, Robert 005, 008, 011, 026, 047, 068, 069, 070,

077, 078, 085, 111, 146

Henretig, Fred 152

Hettinger, Aaron 007

Hinami, Keiki 002

Hitti, E 046

Hoang, Annie 115

Hoffman, Daniel 007

Hofman, Maryann 160

Hogg, Melanie 116

Hon, Stephanie 117

Hughes, Adrienne 008, 011, 026, 047, 068, 069, 070, 077, 078, 111, 146

Huntington, Serena 049, 125, 144

Jacobs, Jr., John 040

Janetto, Paul 116

Jang, David 119, 142

Janke, Alexander 012, 013

Johnson-Arbor, Kelly 159

Jontz, Ashley 084

Kammel, Kevin 050

Kang, A. 059

Kao, Louise 016

Karshenas, Dana 118, 139, 140, 141, 154, 155, 156

Katz, Kenneth 029

Kazzi, Ziad 022, 025, 031, 046, 113, 132, 133, 153

Kearney, Tom 120

Kessler, Benjamin 067

Khalid, Muhammad 086

Kiernan, Emily 033, 053, 117, 132, 153

Kieszak, Stephanie 061

Kim, Eungjae 003, 133

King, Andrew 099, 148, 149

King, Taylore 081

Kirschner, Ron 060, 065, 066

Kleiman, Richard 082

Kleinschmidt, Kurt 006, 098

Koch, David 003
Konchak, Juleigh 009

Koons, Andrew 029

Kopec, Kathryn 113

Kowalski, J 035

Krasowski, Matthew 087

Kreisler, Rachel 084

Krueger, James 035

Kumar, Susheel 054

Kunzler, Nathan 128

Kwai, Kim 044, 124

LaFountain, Nicholas 064, 104

Law, Royal 061

Layer, Mark R 033, 053, 117, 132, 153

Lebin, Jacob 041, 042

Lee, Rosiland 055

Lee, Samuel 161

Lee, Vincent 067

Levin, Bonnie 007

Levine, Michael 001, 084

Lewis, Trevor 122

Liebelt, Erica 027

Liss, David 102

Logan, Barry 057

Lopez, Annette 126, 127

Love, Jennifer 010, 018, 019, 119, 142

LoVecchio, Frank 084

Lutfi, Riad 016

Lynch, Michael 090, 094, 110, 151

Ma, Anita 145

Madsen, James 143

Maher, Patrick 131

Majlesi, Nima 067

Malaeb, M 046

Maloney, Gerald 062, 123

Manini, Alex 036, 109, 114, 131

Marino, Ryan 030, 043, 051, 063, 064, 089, 090, 103, 104, 110, 151

Marsden, Granger 002

Marvel-Coen, James 055

Matus, Mariana 074

Mazer-Amirshahi, Maryann 007, 014, 071, 112

Mazor, Suzan 041

McCabe, Kristen 004

McCormack, Ross 051

McDowell, Caitlin 102

McIntyre, Iain 032

McKay, Charles 143, 154, 155, 156

McKeever, Rita 050

Meggs, William 129, 158, 161

Menendez, Anelle 006, 098

Mesloh, Miranda 060, 065

Micciche, Andrew 104

Middleberg, Robert 052

Miller, Simone 114

Minns, Alicia 023, 048

Mneimneh, Zeina 031, 046

Moore, Elizabeth 136

Moran, Tim 003, 022, 058, 117, 133

Morgan, Brent 003, 058, 133 
Morhaime, Jacquelyn 032

Moss, Michael 045, 069

Mostafa, Ahmed Mamdouh Taha 050, 073, 086, 092

Mozeika, Alex 108

Mrad, Sandra 031

Mudan, Anita 142

Murphy, Christine 101, 116

Murphy, David 042

Murphy, Lauren 008, 011, 026, 047, 068, 069, 070, 077, 078, 111, 146

Murray, Brian 003, 022, 025, 039, 058, 117, 133

Mycyk, Mark 119, 122

Nacca, Nicholas 021

Nelson, Lewis 038, 080, 108

Nemanich, Antonia 027

Nguyen, Elizabeth 154, 155, 156

Noble, Matthew 005, 085

Nogar, Joshua 075, 079

Nogee, Daniel 057

O'Donnell, Katherine 150

Offerman, Steve 001

Okawa, Kent 136

Osterhoudt, Kevin 004, 152, 160

Otter, Jenna 032

Ovakim, Daniel 024

Overbeek, Daniel 012, 013

Owen, Kelly 044

Padilla-Jones, Angela 001, 059

Palmer, Katherine 041

Parker Cote, Jennifer 129, 158, 161

Patel, Mayur 082

Peck, Edwin 105

Perrone, Jeanmarie 010, 014, 018, 019

Phillips, Kelly 024

Phillips, Todd 094, 097

Pizon, Anthony 087, 090, 103, 110, 118, 138, 139, 140, 141

Pluckrose, Dawn 095

Polsky, Tracey 004

Porter, Lauren 134

Prather, Colin 026, 111

Punja, Mohan 082

Quinn, Nicholas 107

Radhakrishnan, Rupa 016

Ralston, Samuel 133, 143

Ramirez, Margaret 066

Rasin, Arkady 002, 009, 015

Rasmussen, Marcia 060, 065

Ratprasert, Natthasiri 081

Rianprakaisang, Tony 008, 011, 026, 047, 068, 069, 070, 077, 078, 111, 146

Richardson, Lynne 036, 109

Riddle, Matthew 023

Riederer, Anne 071, 112

Ritchie, James 003

Rivara, Michael 095

Rizvanolli, Lum 002, 009, 015

Rodriguez, Abraham 028

Ross, Bryan 021

Roszell, Laurie 143

Roth, Brett 006, 098
Routsolias, Joanne 122

Ruck, Bruce 038, 080, 108

Ruha, Anne-Michelle 001, 134

Rurup, Edmund 080

Rymer, Jacqueline 087

Sabbatini, Amber 027

Sahin, Aynur 031, 132

Saikumar, Hairitha 028

Santos, Cynthia 038, 113

Sarker, Abeed 018, 019

Scarth, Caleb 015

Scheerlinck, Pieter 091

Schier, Joshua 061

Schindler, Joanna 003

Schulte, Joann 006, 098

Scoccimarro, Anthony 043, 087, 089, 090, 103, 110, 151

Shah, Aakash 157

Shah, Kartik 100, 101

Shank, Brandon 006

Shao, Shirley 043, 089, 090, 103, 110, 151

Sharma, Navneet 054

Shastry, Siri 036

Shively, Rachel 075

Shofer, Frances 010

Shulman, Joshua 063, 089, 104, 110

Shusterman, Dennis 115

Sidlak, Alexander 030, 043, 063, 089, 090, 103, 110, 151

Silver, Elizabeth 033, 153

Simpson, Serge 017

Singh, Surjit 054

Singla, Veena 115

Skaugen, John 087

Smedley, Lucas 028

Smith, Eric 006, 098

Smith, Karen 060, 065, 066

Smith, Lynette 060, 065

Smollin, Craig 120

Snow, Jerry 059

Spyres, Meghan 105, 112, 136

Srisuma, Sahaphume 081, 137

Steck, Alaina 072, 117

Stickley, Carolyn 142

Surmaitis, Ryan 029

Tamama, Kenichi 051, 087

Tarabar, Asim 057

Taub, Emily 131

Taylor, Garrett 094

Taylor, Taryn 053

Thomas, Stephen 001

Thompson, John 126

Tomassoni, Anthony 057

Tortora, Laura 037, 096

Touma, Loren 017

Trakulsrichai, Satariya 081, 137

Tweet, Marit 130

V, Vamshi 054

Vearrier, David 073, 092, 160

Vedanthan, Rajesh 109 
Veltre, Michael 038

Vo, Kathy 145

Vohra, Rais 049, 113, 125, 144

Wahl, Michael 076, 130

Walsh, Steven 017, 035

Wananukul, Winai 137

Wang, Bo 114

Watsjold, Bjorn 042

Watts, Darius 053

Wax, Paul 071, 112, 114, 118, 138, 139, 140, 141, 143, 154, 155, 156

Webb, Cynthia 007

Wegman, Kevin 143

Weiss, Sarah 017, 035

Wermuth, Mary 040

Wiegand, Timothy 020, 021
Williams, Austin 125

Wittler, Mary 135

Wong, Anselm 113

Wongvisavakorn, Sunun 081

Wosiski-Kuhn, Marlena 135

Wu, Ming Li 128

Wymer, Karisa 125

Yancey, Arthur 058

Yang, Chen-Chang 128

Yanta, Joseph 030, 051, 103, 110

Yee, Noa 084

Yeo, Jonathan 036

Zamanpour, Elmira 144

Zhou, Victoria 142 